\title{
Supporting Information for: \\ Ketone Synthesis by a Nickel-Catalyzed Dehydrogenative Cross-Coupling of Primary Alcohols
}

\author{
Thomas Verheyen, ${ }^{\dagger \neq}$ Lars van Turnhout, ${ }^{\dagger \#}$ Jaya Kishore Vandavasi, ${ }^{\dagger \#}$ Eric S. Isbrandt, ${ }^{\dagger}$ \\ Wim M. De Borggraeve, ${ }^{\ddagger}$ Stephen G. Newman $*, \dagger$ \\ ${ }^{\dagger}$ Centre for Catalysis Research and Innovation, Department of Chemistry and \\ Biomolecular Sciences, University of Ottawa, Ottawa, Ontario, Canada, K1N 6N5 \\ ${ }^{*}$ Molecular Design and Synthesis, Department of Chemistry, KU Leuven, 3001 Leuven, \\ Belgium \\ *Email: stephen.newman@uottawa.ca
}

\section{Table of Contents}

1. General considerations

2. Optimization

S4

3. Reaction scope

4. Mechanistic studies

S30

5. cHPLC and SFC data

S36

6. NMRs

S38

7. References

S86 


\section{$\underline{\text { 1. General considerations }}$}

\subsection{General experimental details}

Unless otherwise indicated, reactions were conducted under an atmosphere of nitrogen in $8 \mathrm{~mL}$ screw-capped vials that were oven dried $\left(120{ }^{\circ} \mathrm{C}\right)$. Column chromatography was performed manually using Silicycle F60 40-63 $\mu \mathrm{m}$ silica gel. Analytical thin layer chromatography (TLC) was conducted with aluminum-backed EMD Millipore Silica Gel $60 \mathrm{~F}_{254}$ pre-coated plates. Visualization of developed plates was performed under UV light $(254 \mathrm{~nm})$ and/or using $\mathrm{KMnO}_{4}$.

\subsection{Instrumentation}

${ }^{1} \mathrm{H}$ NMR and ${ }^{13} \mathrm{C}$ NMR were recorded on a Bruker AVANCE $400 \mathrm{MHz}$ spectrometer. ${ }^{1} \mathrm{H}$ NMR spectra were internally referenced to the residual solvent signal (e.g., $\mathrm{CDCl}_{3}=7.27$ ppm). ${ }^{13} \mathrm{C}$ NMR spectra were internally referenced to the residual solvent signal (e.g., $\left.\mathrm{CDCl}_{3}=77.00 \mathrm{ppm}\right)$. Data for ${ }^{1} \mathrm{H}$ NMR are reported as follows: chemical shift $(\delta \mathrm{ppm})$, multiplicity $(\mathrm{s}=$ singlet, $\mathrm{d}=$ doublet, $\mathrm{t}=$ triplet, $\mathrm{q}=$ quartet, $\mathrm{m}=$ multiplet $)$, coupling constant $(\mathrm{Hz})$, integration. IR spectra were obtained using a Nicolet6700 FT-IR spectrometer with a diamond ATR crystal (ThermoScientific) and are reported in terms of frequency of absorption $\left(\mathrm{cm}^{-1}\right)$. GC yields for optimization studies were obtained via a 5-point calibration curve using FID analysis on an Agilent Technologies 7890B GC with $30 \mathrm{~m} \times 0.25 \mathrm{~mm}$ HP-5 column. Accurate mass data (EI) was obtained from an Agilent 5977A GC/MSD using MassWorks 4.0 from CERNO Bioscience. ${ }^{1}$ HRMS-ESI data was obtained from a Micromass Q-TOF 2 quadrupole - time-of-flight mass spectrometer with ESI source.

\subsection{Materials}

Organic solvents were purified by rigorous degassing with nitrogen before passing through a PureSolv solvent purification system. Low water content was confirmed by Karl Fischer titration ( $<25 \mathrm{ppm}$ for all solvents). Unless otherwise noted, reagents were used as received. $\mathrm{Ni}(\mathrm{cod})_{2}$, quinuclidine $(97 \%), \mathrm{NEt}_{3}$, and phenol were obtained from Sigma Aldrich. Triphos (97\%) and PMP (97\%) were obtained from Alfa Aesar. TMP 
(99\%) was obtained from Sigma Aldrich. Acetone was dried prior to use over $\mathrm{CaSO}_{4}$ for 48 hours. After filtration, acetone was stored on $\mathrm{K}_{2} \mathrm{CO}_{3}$ in a nitrogen-filled glovebox. Most organotriflates are prepared according to the procedure of Goossen and co-workers (Scheme S1). ${ }^{2}$ Tyrosine triflate was prepared and purified according to the procedure of Shieh and Carlson. ${ }^{2}$ 4-Acetamidophenyl trifluoromethanesulfonate was prepared and purified according to the procedure of Kapur and co-workers. ${ }^{3}$

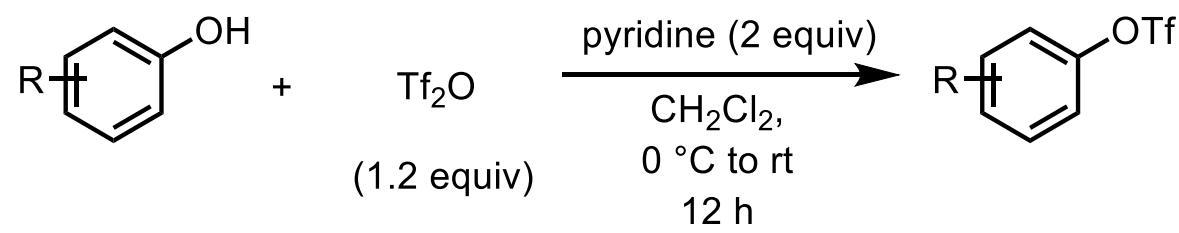

Scheme S1. General method for the synthesis of aryl triflates. ${ }^{2}$

General procedure for the synthesis of aryl triflates. A solution of trifluoromethanesulfonic anhydride $(4.00 \mathrm{~mL}, 24.0 \mathrm{mmol})$ in $\mathrm{CH}_{2} \mathrm{Cl}_{2}(10.0 \mathrm{~mL})$ was added dropwise to a solution of pyridine $(3.23 \mathrm{~mL}, 40.0 \mathrm{mmol})$ and the corresponding phenol $(20.0 \mathrm{mmol})$ in anhydrous $\mathrm{CH}_{2} \mathrm{Cl}_{2}(20 \mathrm{~mL})$ at $0{ }^{\circ} \mathrm{C}$. After complete addition, the mixture was warmed to room temperature and allowed to stir for 12 hours. The mixture was then diluted with DCM and quenched with $10 \%$ aq. $\mathrm{HCl}$. The organic layer was separated, washed with brine, and dried over $\mathrm{Na}_{2} \mathrm{SO}_{4}$. The solvent was removed under reduced pressure and the residue was purified by column chromatography to give the corresponding organotriflates. 


\section{Optimization}

\subsection{General procedure for optimization tables}

Inside the glove box, to an $8 \mathrm{~mL}$ screw-capped reaction vial equipped with a magnetic stir bar, $\mathrm{Ni}(\operatorname{cod})_{2}$ and ligand were added and dissolved in toluene. Alcohol, organo(pseudo)halide, base and an oxidant were added. The vial was capped, removed from the glove box, and placed in an oil bath pre-heated to respective temperature. After

stirring for 20 hours, the reaction vial was removed from the oil bath and cooled down to room temperature. 1,3,5-trimethoxybenzene was added as an internal standard prior to filtering the reaction over a short pad of silica gel and washing with EtOAc. The filtrate was diluted to an appropriate concentration and analyzed by GC-FID using a 5-point calibration curve to determine the yield.

Reaction outside of glovebox (marked with * in table S2). Reactions with $\mathrm{Ni}(\mathrm{II})$ sources were set up via standard schlenk techniques and $0.3 \mathrm{mmol}$ scale. Solid material $\left(\mathrm{NiX}_{2}\right.$, Triphos and solid reactants) were added to a flame dried schlenk tube. The vial was purged with $\mathrm{N}_{2}$ (3X vacuum/ $\mathrm{N}_{2}$ cycles). Liquid reagents and solvent were degassed with $\mathrm{N}_{2}$ prior to addition. Dry toluene, alcohol, organo(pseudo)halide, TMP and acetone $\left(\mathrm{CaSO}_{4}\right.$ and $\mathrm{K}_{2} \mathrm{CO}_{3}$ dried) were added. The schlenk tube was placed in an oil bath at 130 ${ }^{\circ} \mathrm{C}$ for 20 hours. 1,3,5-trimethoxybenzene was added as an internal standard prior to filtering the reaction over a short pad of silica gel and washing with EtOAc. The filtrate was concentrated and analyzed by ${ }^{1} \mathrm{H} \mathrm{NMR}\left(\mathrm{CDCl}_{3}\right)$ to determine the yield.

Initial conditions for reaction screening were modified from previously developed carbonyl-Heck reaction conditions ${ }^{4}$ with the inclusion of a hydrogen acceptor (5 equiv) and the use of benzyl alcohol in place of a benzaldehyde coupling partner (Table S1). Acetone was found to be the most promising (entry 1), and good yields could be obtained by using the organotriflate in excess with 2 equivalents of TMP (entry 15) 
Table S1. Screening hydrogen acceptors.

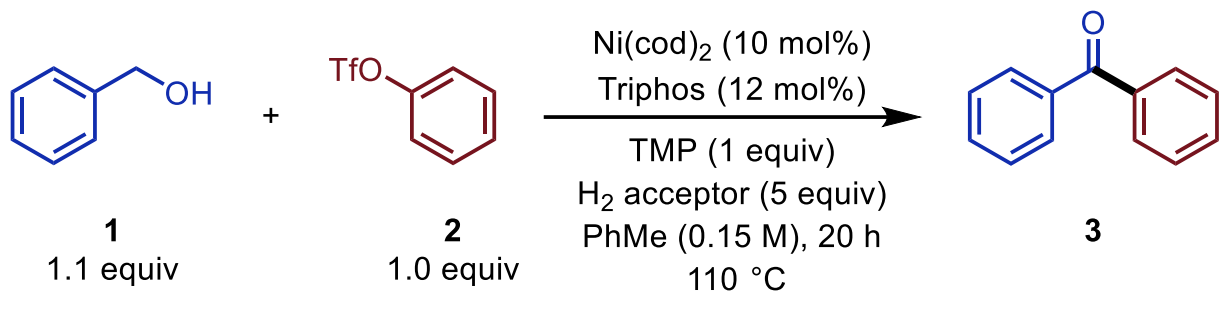

Entry

1

2

3

4

5

6

7

8

9

10

11

12

Pivaldehyde (75\% in $t$ - $\mathrm{BuOH})$

$\mathrm{H}_{2}$ acceptors

Acetone

$\mathrm{PhCOCF}_{3}$

Tetralone

Cyclohexanone

1,2-Dihydronapthalene

Cyclohexene

Styrene

1,4-Benzoquinone

Acetaldehyde

Trifluoroacetone

Ethylpyruvate
Yield

0

13

24

11

23

0

0

31

6

Traces

23

13 Acetone with $\mathrm{PhCH}_{2} \mathrm{OH}$ (1 equiv), PhOTf (1.5 equiv),

52

TMP (1 equiv)

14 Acetone with $\mathrm{PhCH}_{2} \mathrm{OH}$ (1 equiv), PhOTf (1.5 equiv), TMP (2 equiv)

Further optimization was carried out to identify the conditions illustrated below (Table $\mathrm{S} 2$, entry 1). The importance of various parameters, including ligand, concentration, base, solvent, temperatures, stoichiometry and catalyst loading are given below. 
Table S2. Additional optimization experiments.

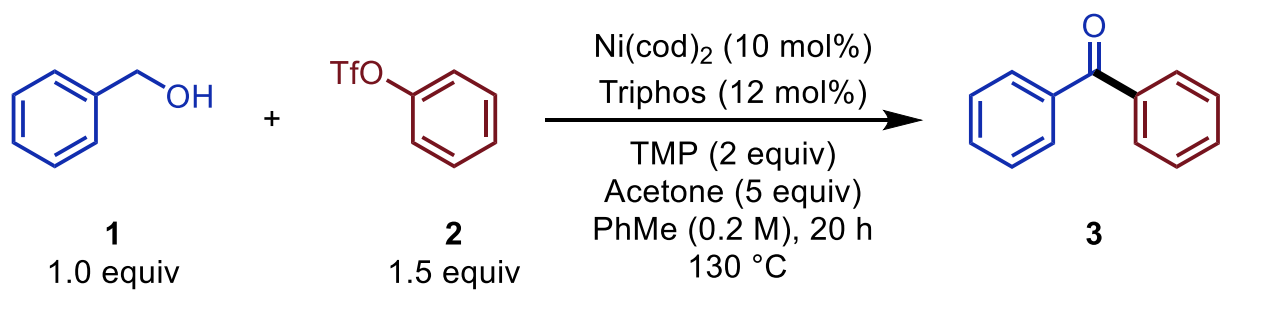

\begin{tabular}{c}
\hline Deviation from standard reaction \\
conditions
\end{tabular}

Yield $3(\%)$

\begin{tabular}{|c|c|c|}
\hline 1 & No deviation & 93 \\
\hline 2 & Ligand $=$ Dppp & 23 \\
\hline 3 & Ligand $=$ Dppf & 19 \\
\hline 4 & Ligand $=$ N-Triphos & 1 \\
\hline 5 & $\begin{array}{c}\text { Ligand = Xantphos, Dcypp, BINAP, S- } \\
\text { Phos, } \mathrm{PPh}_{3}\end{array}$ & Traces \\
\hline 6 & Base $=$ PMP & 90 \\
\hline 7 & Base $=\mathrm{K}_{2} \mathrm{CO}_{3}$ & 64 \\
\hline 8 & Base $=\mathrm{Et}_{3} \mathrm{~N}$ & 76 \\
\hline 9 & Base $=$ Quinuclidine & 56 \\
\hline 10 & Base $=\mathrm{DABCO}$ & 55 \\
\hline 11 & Concentration $=0.3 \mathrm{M}$ & 88 \\
\hline 12 & Concentration $=0.1 \mathrm{M}$ & 87 \\
\hline 13 & $\mathrm{Ni}(\operatorname{cod})_{2} /$ Triphos $=7.5 / 9.0 \mathrm{~mol} \%$ & 84 \\
\hline 14 & $\mathrm{Ni}(\mathrm{cod})_{2} /$ Triphos $=15 / 18 \mathrm{~mol} \%$ & 96 \\
\hline 15 & Solvent $=\mathrm{m}-\mathrm{Xylene}$ & 88 \\
\hline 16 & Solvent $=$ Mesitylene & 86 \\
\hline 17 & Solvent $=\mathrm{NMP}$ & Traces \\
\hline 18 & Solvent $=$ Sulfolane & Traces \\
\hline 19 & $\mathrm{PhCl}, \mathrm{PhBr}$ or $\mathrm{PhI}$ instead of PhOTf & Traces \\
\hline 20 & PhOMs instead of PhOTf & 30 \\
\hline
\end{tabular}




\section{1,1,1-Trifluoroacetone instead of acetone}

$$
1.1 \text { equiv of PhOTf }
$$

$$
\text { Temperature }=110^{\circ} \mathrm{C}
$$

* Reactions set up outside of glovebox according to reported protocol above.

\section{Structures of ligands}<smiles>CC(CPc1ccccc1)(Cc1ccccc1)Cc1ccccc1</smiles>

Triphos<smiles>c1ccc(-c2ccc3ccccc3c2-c2c(P(c3ccccc3)c3ccccc3)ccc3ccccc23)cc1</smiles>

BINAP<smiles>c1ccc(P(CCCP(c2ccccc2)c2ccccc2)c2ccccc2)cc1</smiles><smiles>COc1cccc(OC)c1-c1ccccc1P(C)(=O)O</smiles>

S-Phos<smiles>CC1(C)c2cccc(P)c2Oc2c(P)cccc21</smiles>

Xantphos

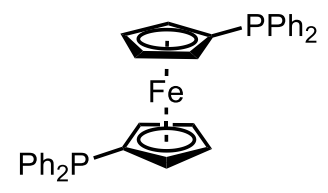

dppf<smiles>C1CCC(P(CCCP(C2CCCCC2)C2CCCCC2)C2CCCCC2)CC1</smiles>

dcypp<smiles>Pc1ccccc1</smiles>

$\mathrm{N}$-Triphos

As illustrated above (Table S2, entry 1 vs entry 29), the reaction of benzyl alcohol with phenyltriflate was found to be efficient at $110{ }^{\circ} \mathrm{C}$. However, $130{ }^{\circ} \mathrm{C}$ was found to be 
more effective for challenging substrates, and even higher temperatures were necessary in some cases (Scheme S2).

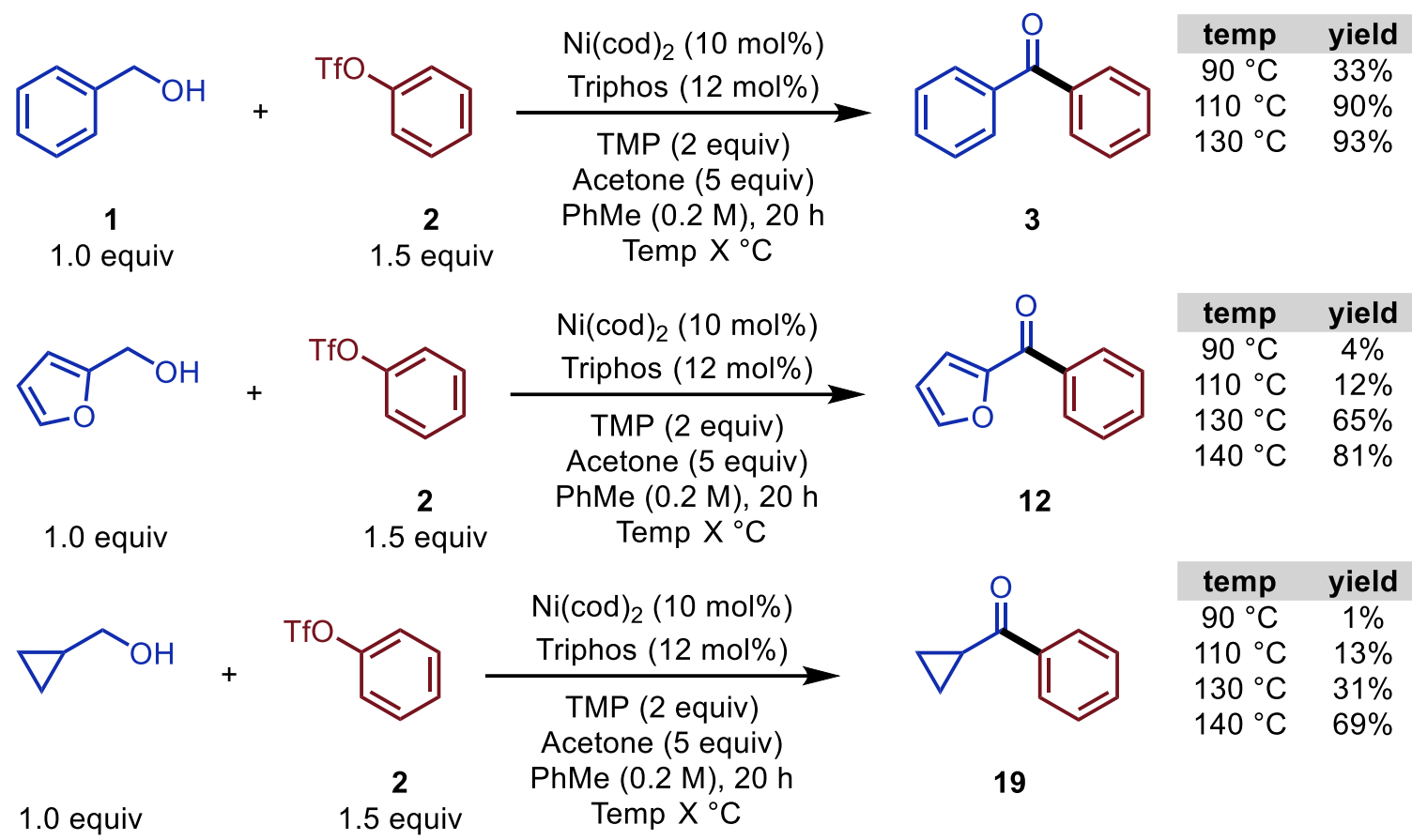

Scheme S2. Screening of temperature for different substrates.

The reaction of benzyl alcohol with phenyltriflate was found to be most efficient with acetone as hydrogen acceptor. However, the reaction also provided moderate yield without acetone, suggesting the organotriflate functions as the oxidant as well. Equivalents of acetone and/or phenyl triflate were changed to investigate the role of both as oxidant (Table S3).

Table S3. Influence of the oxidizing agents acetone and phenyl triflate.

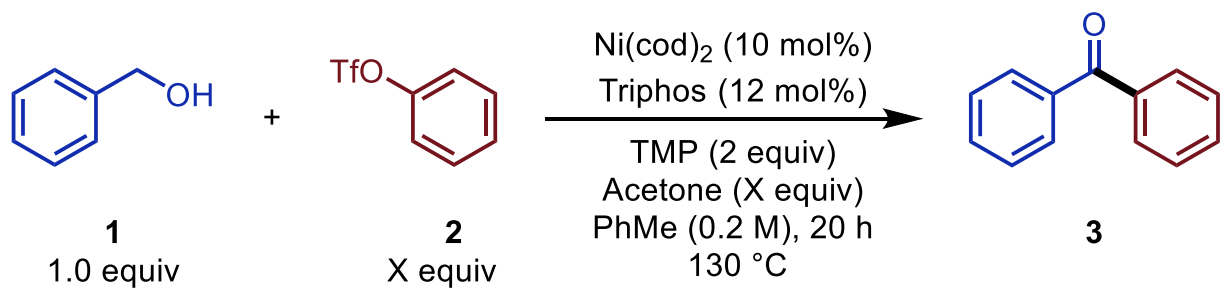

\begin{tabular}{cccc}
\hline entry & equiv PhOTf & equiv Acetone & yield 3 $(\boldsymbol{\%})$ \\
\hline 1 & 1.5 & 5.0 & 93 \\
2 & 1.5 & 0 & 72 \\
3 & 1.1 & 5.0 & 62
\end{tabular}




\begin{tabular}{cccc}
4 & 1.1 & 0 & 52 \\
5 & 2.0 & 5.0 & 99 \\
6 & 2.0 & 0 & 96 \\
\hline
\end{tabular}

\subsection{Troubleshooting}

Some limitations, tips, and tricks for the reaction are provided below.

1. Functional group tolerance: A robustness-type screen was carried out to obtain general information about the functional group tolerance of the reaction. Compatible additives are those that minimally influenced the yield (>75\% product) when present. Compatible yet challenging additives hindered the reaction but significant yield (40-75\% yield) was still obtained. Incompatible additives resulted in low yield $(<40 \%)$.

\section{Compatible additives}

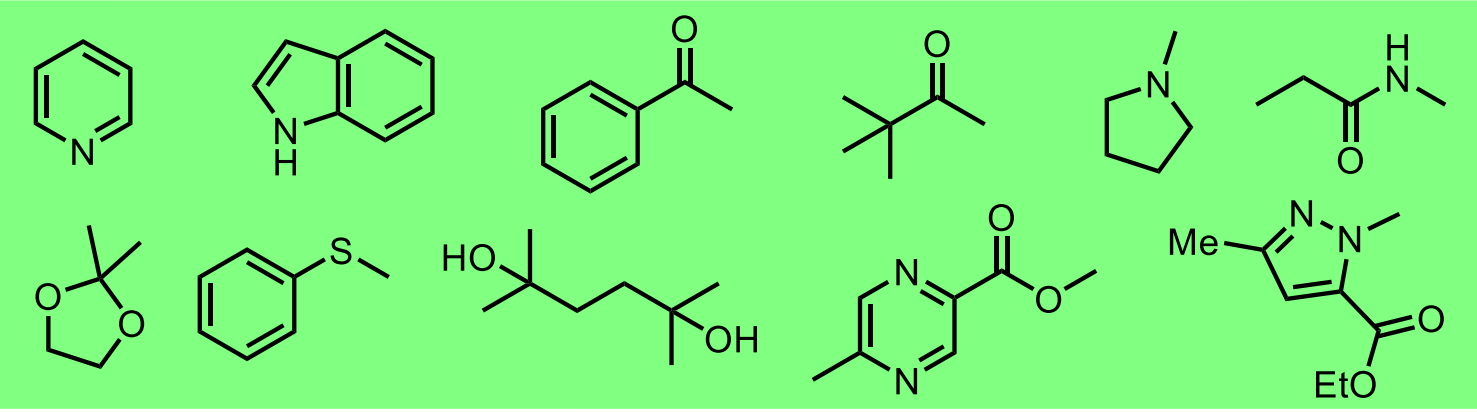

\section{Compatible yet challenging additives}<smiles>Nc1ccccc1</smiles>
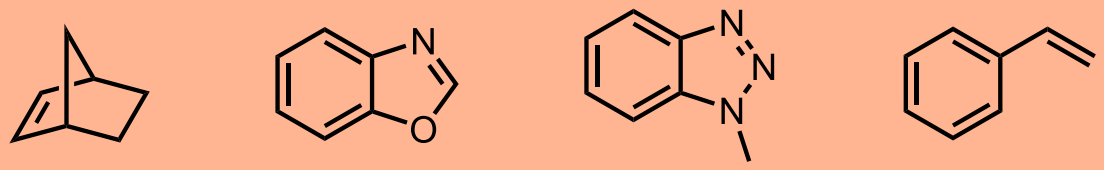

\section{Incompatible additives}

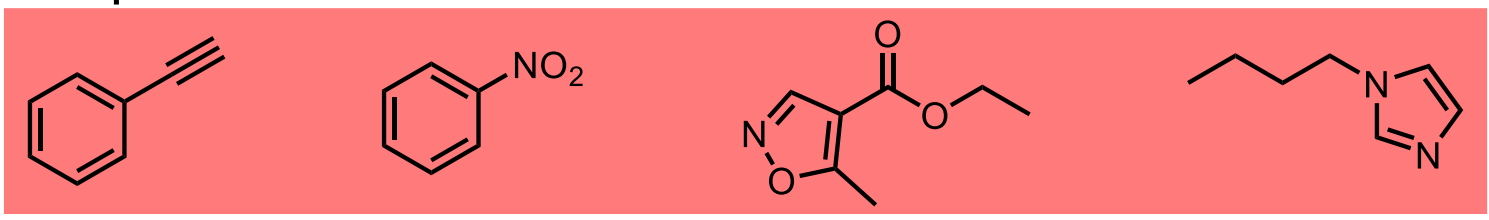

Scheme S3. Robustness screen.

Several alcohols and triflates (Scheme S4) were tested in the coupling without success. Studies are ongoing to find solutions for these problematic substrates. 
$y^{\prime \prime}{ }^{\prime} \sim^{\circ H}$<smiles>CC(O)CCO</smiles><smiles>OCc1nc2ccccc2s1</smiles><smiles>C#CCCCO</smiles><smiles>OCC1CC2C=CC1C2</smiles><smiles>OCc1ccccn1</smiles><smiles>O=C1CC[C@@H](CO)N1</smiles><smiles>CC(=O)NCO</smiles><smiles>OCc1ccc(Br)cc1</smiles><smiles>O=[N+]([O-])c1ccc(CO)cc1</smiles><smiles>Nc1ccc(CO)cc1</smiles><smiles>OCc1ccc(I)cc1</smiles><smiles>OCCN1CCN(CCCN2c3ccccc3Sc3ccc(Cl)cc32)CC1</smiles><smiles>Cc1ncc(C[n+]2csc(CCO)c2C)c(N)n1</smiles>

$\mathrm{MeOH}$<smiles>COc1cccc(I)c1</smiles><smiles>CCOc1ccc(-n2ccnc2)cc1</smiles>

Scheme S4. Unsuccessful alcohol and triflate substrates.

Vinyltriflates gave full conversion of the alcohol however no product selectivity was obtained using different ligands (Triphos, dppp, DIOP, dppf) and bases (TMP, DABCO, $\left.\mathrm{Et}_{3} \mathrm{~N}, \mathrm{NCy}_{2} \mathrm{Me}\right)$. Exemplary reaction shown below.

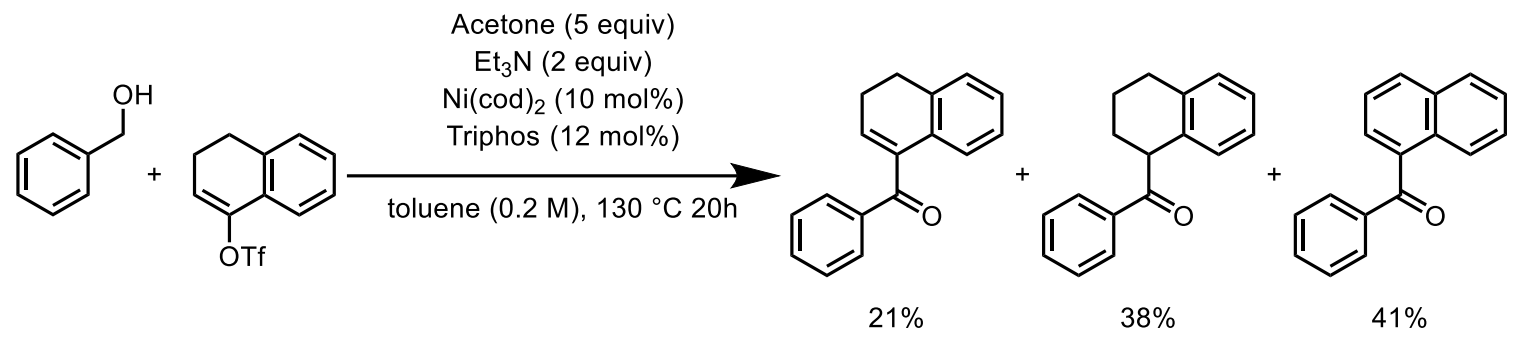

Scheme S5. Unsuccessful vinyltriflate reaction leading to a mixture of products. Yields determined by ${ }^{1} \mathrm{H}$ crude $\mathrm{NMR}$ in $\mathrm{CDCl}_{3}$.

2. Air sensitivity. As is common with $\mathrm{Ni}(0)$ catalysis, the reaction is highly sensitive to oxygen and moisture. All reactions presented were set up in an argon filled glovebox with $<0.2 \mathrm{ppm} \mathrm{O}_{2}$. Vials were sealed with a screw-cap, Teflon-lined septum inside the box prior to being removed and placed in an oil bath. Addition of reagents or solvents 
through the septum outside of the glovebox could lead to a decrease in yield, as will piercing the septum for taking samples for TLC or other analysis.

3. Troubleshooting low yielding reactions. For challenging substrates, we suggest first trying the reaction at a higher temperature and increasing the equivalents of the organotriflate. Increasing the catalyst, ligand, or base equivalencies only had minor impact. Increasing or decreasing the amount of acetone was also found to be generally detrimental, with the exception of coupling substrates bearing multiple primary alcohol functional groups (e.g. synthesis of $\mathbf{1 1}$ ).

4. Price comparison and availability of aldehydes versus alcohols. Many of the ketones synthesized in the manuscript can be alternatively prepared using aldehydes instead of alcohols as starting material, giving a redox-neutral reaction. ${ }^{4}$ In most cases, we recommend using alcohols due to the better availability, price, stability, and in many cases, yields. In particular, aliphatic alcohols are more accessible than the corresponding aldehydes. Some examples of alcohols used in our reaction scope are shown in Scheme S6.

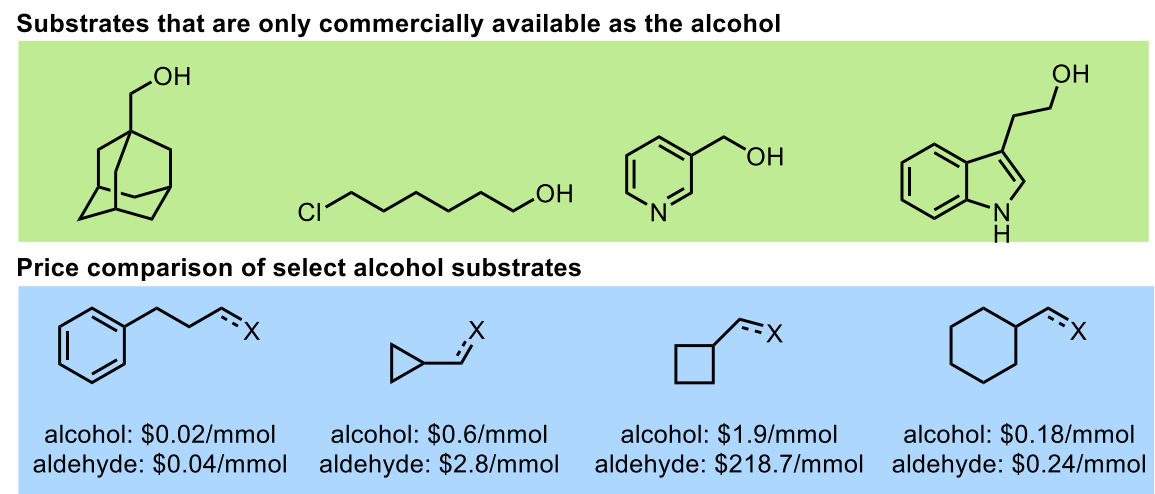

Scheme S6. Comparison of commercial availability of alcohols to the respective aldehydes. Data obtained July 2018 from: https://www.sigmaaldrich.com/canadaenglish.html). Prices given are in Canadian Dollars. 


\section{$\underline{\text { 3. Reaction scope }}$}

\subsection{Synthesis of novel alcohols and organotriflates}<smiles>CCOc1ccc(C(C)(C)CC(C)(C)C)cc1Cc1ccc(Cl)cc1Cl</smiles>

s1

2-(2,4-dichlorobenzyl)-4-(2,4,4-trimethylpentan-2-yl)phenyl

trifluoromethanesulfonate (S1) was prepared and purified according to the general procedure for the preparation of triflates. S1 was obtained as a colorless oil (728 $\mathrm{mg}, 89 \%$ yield). ${ }^{1} \mathbf{H}$ NMR $\left(\mathrm{CDCl}_{3}, 400 \mathrm{MHz}\right) \delta 7.43(\mathrm{~d}, J=2.1 \mathrm{~Hz}, 1 \mathrm{H}), 7.31(\mathrm{dd}, J=8.7,2.5 \mathrm{~Hz}$, $1 \mathrm{H}), 7.22-7.17(\mathrm{~m}, 2 \mathrm{H}), 7.08(\mathrm{~d}, J=2.3 \mathrm{~Hz}, 1 \mathrm{H}), 7.00(\mathrm{~d}, J=8.2 \mathrm{~Hz}, 1 \mathrm{H}), 4.13(\mathrm{~s}, 2 \mathrm{H})$, $1.64(\mathrm{~s}, 2 \mathrm{H}), 1.28(\mathrm{~s}, 6 \mathrm{H}), 0.64(\mathrm{~s}, 9 \mathrm{H}) .{ }^{13} \mathrm{C} \mathrm{NMR}\left(\mathrm{CDCl}_{3}, 100 \mathrm{MHz}\right) \delta$ 150.8, 145.7, 135.2 , 135.0, 133.2, 131.6, 130.4, 129.5, 129.4, 127.2, 126.3, 120.6, 120.2, 117.0, 56.8, 38.5, 33.1, 32.2, 31.7, 31.4. ${ }^{19} \mathbf{F}$ NMR $\left(\mathrm{CDCl}_{3}, 400 \mathrm{MHz}\right) \delta$-73.7. Accurate mass (EI): Theoretical: 496.0854. Found: 496.0769. Spectral Accuracy: 92.4\%. FT-IR: $v\left(\mathrm{~cm}^{-1}\right)$ 2957, 2903, 1588, 1472, 1417, 1247, 1210, 1172, 1139, 1103, 1069, 857, 824, 740, 666, 619.

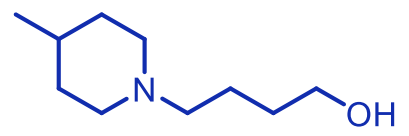

S2

4-(4-methylpiperidin-1-yl)butan-1-ol (S2) In a oven-dried argon flushed roundbottomed flask 4-chlorobutan-1-ol (2.0 g, $18.4 \mathrm{mmol})$, 4-methylpiperidine (2.6 ml, 22.1 $\mathrm{mmol})$, NaI (0.3 g, $2.0 \mathrm{mmol})$ and $\mathrm{K}_{2} \mathrm{CO}_{3}(3.8 \mathrm{~g}, 27.6 \mathrm{mmol})$ were dissolved in Acetone $(12 \mathrm{~mL})$. Reaction mixture was refluxed overnight. After cooling the inorganic salts were filtered off. The filtrate was concentrated under reduced pressure and heptane was added to the concentrated residue. The impurities were filtered off and the filtrate was concentrated under reduced pressure to obtain $\mathbf{S 2}$ as a colourless oil (2.1 g, 66\% yield). ${ }^{1} \mathbf{H}$ NMR $\left(\mathrm{CDCl}_{3}, 400 \mathrm{MHz}\right) \delta 3.68-3.54(\mathrm{~m}, 2 \mathrm{H}), 2.94(\mathrm{~d}, J=11.8 \mathrm{~Hz}, 2 \mathrm{H}), 2.34-2.32$ $(\mathrm{m}, 2 \mathrm{H}), 1.98-1.92(\mathrm{~m}, 2 \mathrm{H}), 1.67-1.64(\mathrm{~m}, 6 \mathrm{H}), 1.41-1.25(\mathrm{~m}, 3 \mathrm{H}), 0.91(\mathrm{~d}, J=6.3 \mathrm{~Hz}$, $3 \mathrm{H}) .{ }^{13} \mathrm{C}$ NMR $\left(\mathrm{CDCl}_{3}, 100 \mathrm{MHz}\right) \delta$ 62.7, 58.8, 53.8, 33.8, 32.9, 30.8, 26.0, 21.6. Accurate mass (EI): Theoretical: 171.1623. Found: 171.1604. Spectral Accuracy: 96.3\%. FT-IR: $v\left(\mathrm{~cm}^{-1}\right)$ 3367, 3192, 3130, 2919, 2867, 2805, 1452, 1373, 1259, 1154, $1125,1014,974,902,811,772,747,644,541$. 


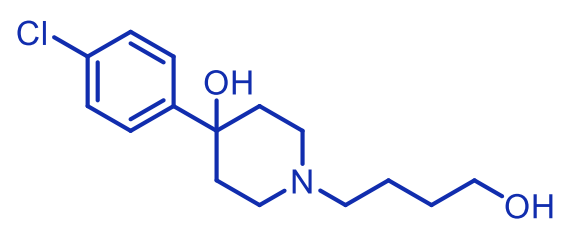

\$3

4-(4-chlorophenyl)-1-(4-hydroxybutyl)piperidin-4-ol (S3) A dry vial was filled with 4(4-chlorophenyl)piperidin-4-ol (1.2 g, $5.6 \mathrm{mmol})$ and $\mathrm{KI}(5.8 \mathrm{mg}, 0.04 \mathrm{mmol})$. Dry toluene $(7.0 \mathrm{~mL})$ and 4-chloro-1-butanol $(368 \mu \mathrm{l}, 3.5 \mathrm{mmol})$ were added. The mixture was refluxed for $48 \mathrm{~h}$, cooled in an ice bath, the precipitate was filtered off and washed twice with distilled $\mathrm{H}_{2} \mathrm{O}$ and cold $\mathrm{Et}_{2} \mathrm{O}$ (cooled in ice bath). After drying, $\mathbf{S 3}$ was obtained as a white powder $(381 \mathrm{mg}, 38 \%) .{ }^{1} \mathbf{H}$ NMR (DMSO-d $\left.6,400 \mathrm{MHz}\right) \delta 7.48(\mathrm{~d}, J$ $=8.6 \mathrm{~Hz}, 2 \mathrm{H}), 7.35(\mathrm{~d}, J=8.6 \mathrm{~Hz}, 2 \mathrm{H}), 4.87$ (s, 1H), 4.62 (br.s, $1 \mathrm{H}), 3.40$ (t, $J=6.0 \mathrm{~Hz}$, $2 \mathrm{H}), 2.64(\mathrm{~d}, \mathrm{~J}=10.8 \mathrm{~Hz}, 2 \mathrm{H}), 2.36-2.28(\mathrm{~m}, 4 \mathrm{H}), 1.87(\mathrm{td}, J=12.9,4.2 \mathrm{~Hz}, 2 \mathrm{H}), 1.56$ (d, $J=12.0 \mathrm{~Hz}, 2 \mathrm{H}), 1.49-1.42(\mathrm{~m}, 4 \mathrm{H}) .{ }^{13} \mathrm{C}$ NMR $\left(\right.$ DMSO-d $\left._{6}, 100 \mathrm{MHz}\right) \delta 149.6,131.2$, 128.2 , 127.3 , 70.0, 61.3, 58.5, 49.5, 38.4, 31.3, 23.9. Accurate mass (EI): Theoretical: 283.1339. Found: 283.1334. Spectral Accuracy: 93.6\%. FT-IR: $v\left(\mathrm{~cm}^{-1}\right)$ 3266, 3118, 2929, 2830, 2630, 1576, 1485, 1415, 1368, 1315, 1227, 1190, 1136, 1086, 1043. Mp: $138-140{ }^{\circ} \mathrm{C}$.

\subsection{General reaction procedure}

Inside the glove box (used for all products 3-44), to an $8 \mathrm{~mL}$ screw-capped reaction vial equipped with a magnetic stir bar, $\mathrm{Ni}(\operatorname{cod})_{2}(0.03 \mathrm{mmol}, 10 \mathrm{~mol} \%)$ and Triphos $(0.036$ mmol, $12 \mathrm{~mol} \%)$ were added and dissolved in toluene $(1.5 \mathrm{~mL}, 0.2 \mathrm{M})$. Alcohol (0.3 mmol $)$, organotriflate $(0.45 \mathrm{mmol})$, TMP $(0.6 \mathrm{mmol})$ and acetone $(1.5 \mathrm{mmol})$ were added. The vial was capped, removed from the glove box, and placed in an oil bath pre-heated to $130{ }^{\circ} \mathrm{C}$. After stirring for 20 hours, the reaction vial was removed from oil bath and cooled down to room temperature. The reaction mixture was diluted with EtOAc transferred to a round bottomed flask, silica was added and the solvent was removed under reduced pressure. The crude was purified by column chromatography.

Reaction outside of glovebox protocol (used for products $\mathbf{3}$ and $\mathbf{5}$ as comparisson): $\mathrm{Ni}(\mathrm{OTf})_{2}(0.03 \mathrm{mmol}, 10 \mathrm{~mol} \%)$, Triphos $(0.036 \mathrm{mmol}, 12 \mathrm{~mol} \%)$ and solid reactant (example 4-methylbenzylalcohol $(0.3 \mathrm{mmol}))$ were added to a flame dried Schlenk tube. The vial was purged with $\mathrm{N}_{2}\left(3 \mathrm{X}\right.$ vacuum/ $\mathrm{N}_{2}$ cycles). Liquid reagents were degassed with $\mathrm{N}_{2}$ prior to addition. Dry toluene $(1.5 \mathrm{~mL}, 0.2 \mathrm{M})$, alcohol $(0.3 \mathrm{mmol})$, organotriflate 
(0.45 mmol), TMP (0.6 mmol) and acetone (1.5 mmol, $\mathrm{CaSO}_{4}$ and $\mathrm{K}_{2} \mathrm{CO}_{3}$ dried) were added. The Schlenk tube was placed in an oil bath at $130{ }^{\circ} \mathrm{C}$ for 20 hours. The reaction mixture was diluted with EtOAc transferred to a round bottomed flask, silica was added and the solvent was removed under reduced pressure. The crude was purified by column chromatography.

\subsection{Reaction scope \& characterization data}<smiles>O=C(c1ccccc1)c1ccccc1</smiles>

benzophenone (3) was prepared according to the general procedure. Purification was done by column chromatography. The fractions were collected using a gradient of $1 \%$ to $7 \%$ EtOAc in hexane to afford 3 as white solid (49.5 mg, 91\% yield). Characterization data matched those previously reported. ${ }^{4}{ }^{1} \mathbf{H}$ NMR $\left(\mathrm{CDCl}_{3}, 400 \mathrm{MHz}\right) \delta 7.82-7.80(\mathrm{~m}$, 4H), 7.61-7.57 (m, 2H), 7.50-7.47 (m, 4H). ${ }^{13} \mathbf{C ~ N M R ~}\left(\mathrm{CDCl}_{3}, 100 \mathrm{MHz}\right) \delta 196.9,137.7$, $132.5,130.2,128.4$.

Also performed with $\mathrm{Ni}(\mathrm{OTf})_{2}$ outside of a glovebox (36.6 mg, 67\% yield).<smiles>O=C(c1ccccc1)c1ccc2ccccc2c1</smiles>

2-benzoylnaphthalene (4) was prepared according to the general procedure from naphthalen-2-ylmethanol and phenyltriflate. Purification was done by column chromatography. The fractions were collected using a gradient of $3 \%$ to $5 \%$ EtOAc in hexane to afford 4 as a white solid $(57.9 \mathrm{mg}, 83 \%$ yield). Characterization data matched those previously reported. ${ }^{5}{ }^{1} \mathbf{H}$ NMR $\left(\mathrm{CDCl}_{3}, 400 \mathrm{MHz}\right) \delta 8.28(\mathrm{~s}, 1 \mathrm{H}), 7.96-7.91(\mathrm{~m}$, 4H), 7.89-7.86 (m, 2H), 7.65-7.60 (m, 2H), 7.58-7.51 (m, 3H). ${ }^{13} \mathbf{C ~ N M R ~}\left(\mathrm{CDCl}_{3}, 100\right.$ $\mathrm{MHz}) \delta 196.7,137.9,135.2,134.8,132.4,132.2$, 131.8, 130.1, 129.4, 128.3, 128.3, $128.3,126.8,125.8$. 
<smiles>Cc1ccc(C(=O)c2ccccc2)cc1</smiles>

4-methylbenzophenone (5) was prepared according to the general procedure. Purification was done by column chromatography. The fractions were collected using a gradient of $1 \%$ to $5 \%$ EtOAc in hexane to afford 5 as a white solid (52.9 mg, $90 \%$ yield). Characterization data matched those previously reported. ${ }^{5} \mathbf{1} \mathbf{H} \mathbf{N M R}\left(\mathrm{CDCl}_{3}, 400 \mathrm{MHz}\right) \delta$ 7.81-7.78 (m, 2H), $7.74(\mathrm{~d}, J=8.2 \mathrm{~Hz}, 2 \mathrm{H}), 7.58(\mathrm{tt}, J=7.4,1.3 \mathrm{~Hz}, 1 \mathrm{H}), 7.48(\mathrm{t}, J=7.8$ $\mathrm{Hz}, 2 \mathrm{H}), 7.29$ (d, $J=7.9 \mathrm{~Hz}, 2 \mathrm{H}), 2.45(\mathrm{~s}, 3 \mathrm{H}) \cdot{ }^{13} \mathbf{C} \mathbf{N M R}\left(\mathrm{CDCl}_{3}, 100 \mathrm{MHz}\right) \delta 196.5$, $143.2,137.9,134.8,132.1,130.3,129.9,128.9,128.2,21.6$.

Also performed with $\mathrm{Ni}(\mathrm{OTf})_{2}$ outside of a glovebox (40.0 mg, 68\% yield).<smiles>COc1ccc(C(=O)c2ccccc2)cc1</smiles>

4-methoxybenzophenone (6) was prepared according to the general procedure from (4methoxyphenyl)methanol and phenyltriflate. Purification was done by column chromatography. The fractions were collected using a gradient of $0 \%$ to $2 \%$ EtOAc in hexane to afford 6 as a colorless oil (61 mg, 96\% yield). Characterization data matched those previously reported. ${ }^{4} \mathbf{H}$ NMR $\left(\mathrm{CDCl}_{3}, 400 \mathrm{MHz}\right) \delta 7.86-7.82(\mathrm{~m}, 2 \mathrm{H}), 7.78-7.75$ $(\mathrm{m}, 2 \mathrm{H}), 7.57(\mathrm{tt}, J=6.6,1.3 \mathrm{~Hz}, 1 \mathrm{H}), 7.49-7.46(\mathrm{~m}, 2 \mathrm{H}), 6.99-6.96(\mathrm{~m}, 2 \mathrm{H}), 3.90$ (s, 3H). ${ }^{13} \mathrm{C}$ NMR $\left(\mathrm{CDCl}_{3}, 100 \mathrm{MHz}\right) \delta 195.5,163.2,138.2,132.5,131.8,130.1,129.7$, $128.1,113.5,55.5$.<smiles>COc1cccc(C(=O)c2ccccc2)c1</smiles>

3-methoxybenzophenone (7) was prepared according to the general procedure. Purification was done by column chromatography. The fractions were collected using a gradient of $0 \%$ to $4 \%$ EtOAc in hexane to afford 7 as a colorless oil (58 mg, 91\% yield). Characterization data matched those previously reported. ${ }^{6} \mathbf{H} \mathbf{~ N M R}\left(\mathrm{CDCl}_{3}, 400 \mathrm{MHz}\right) \delta$ 7.82-7.80 (m, 2H), 7.59-7.57 (m, 1H), 750-7.46 (m, 2H), 7.40-7.33 (m, 3H), 7.15-7.12 $(\mathrm{m}, 1 \mathrm{H}), 3.86(\mathrm{~s}, 3 \mathrm{H}) .{ }^{13} \mathbf{C} \mathbf{~ N M R}\left(\mathrm{CDCl}_{3}, 100 \mathrm{MHz}\right) \delta 196.5,159.6,138.9,137.6,132.4$, $130.0,129.2,128.3,122.9,118.9,114.3,55.5$. 
<smiles>O=C(c1ccccc1)c1cccc2c1OCO2</smiles>

benzo $[d][1,3]$ dioxol-4-yl(phenyl)methanone (8) was prepared according to the general procedure. Purification was done by column chromatography. The fractions were collected using a gradient of $10 \%$ to $20 \%$ EtOAc in hexane to afford $\mathbf{8}$ as a colorless oil (59.9 mg, 88\% yield). ${ }^{1} \mathbf{H}$ NMR $\left(\mathrm{CDCl}_{3}, 400 \mathrm{MHz}\right) \delta$ 7.87-7.84 (m, 2H), 7.60 (tt, $J=7.4$, $1.3 \mathrm{~Hz}, 1 \mathrm{H}), 7.50-7.46(\mathrm{~m}, 2 \mathrm{H}), 7.12$ (dd, $J=7.7,1.3 \mathrm{~Hz}, 1 \mathrm{H}), 7.00$ (dd, $J=7.7,1.3 \mathrm{~Hz}$, $1 \mathrm{H}), 6.91(\mathrm{t}, J=5.9 \mathrm{~Hz}, 1 \mathrm{H}), 6.02(\mathrm{~s}, 2 \mathrm{H}) .{ }^{13} \mathbf{C ~ N M R}\left(\mathrm{CDCl}_{3}, 100 \mathrm{MHz}\right) \delta 193.3,148.4$, 147.0, 137.6, 132.8, 129.8, 128.3, 123.0, 121.3, 120.6, 111.7, 101.6. Accurate mass (EI): Theoretical: 226.0630. Found: 226.0624. Spectral Accuracy: 99.5\%. FT-IR: v $\left(\mathrm{cm}^{-1}\right)$ 3064, 2897, 1654, 1594, 1499, 1445, 1367, 1281, 1244, 1193, 1159, 1061, 929, $863,827,772,729,703,673$.<smiles>O=C(c1ccccc1)c1ccc(C(F)(F)F)cc1</smiles>

4-(trifluoromethyl)benzophenone (9) was prepared according to the general procedure from (4-(trifluoromethyl)phenyl)methanol and phenyltriflate. Purification was done by column chromatography. The fractions were collected using a gradient of $2 \%$ to $6 \%$ EtOAc in hexane to afford 9 as a white solid $(60.8 \mathrm{mg}, 81 \%$ yield). Characterization data matched those previously reported. ${ }^{5}{ }^{1} \mathbf{H}$ NMR $\left(\mathrm{CDCl}_{3}, 400 \mathrm{MHz}\right) \delta 7.92-7.89(\mathrm{~m}, 2 \mathrm{H})$, 7.83-7.80 (m, 2H), 7.78-7.75 (m, 2H), 7.64 (tt, $J=7.4,1.3 \mathrm{~Hz}, 1 \mathrm{H}), 7.54-7.50(\mathrm{~m}, 2 \mathrm{H})$. ${ }^{13} \mathbf{C ~ N M R}\left(\mathrm{CDCl}_{3}, 100 \mathrm{MHz}\right) \delta 195.5,140.8,136.8,133.9,133.6,133.1,130.1,130.1$, $128.5,125.4,125.4,125.3,125.3,125.0,122.3$.<smiles>O=C(c1ccccc1)c1ccc(F)cc1</smiles>

4-fluorobenzophenone (10) was prepared according to the general procedure. Purification was done by column chromatography. The fractions were collected using a gradient of $0 \%$ to $5 \%$ acetone in hexane to afford $\mathbf{1 0}$ as a white solid $(42.0 \mathrm{mg}, 70 \%$ yield). Characterization data matched those previously reported. ${ }^{5}{ }^{1} \mathbf{H} \mathbf{~ N M R}\left(\mathrm{CDCl}_{3}, 400\right.$ $\mathrm{MHz}) \delta 7.88-7.83(\mathrm{~m}, 2 \mathrm{H}), 7.80-7.78(\mathrm{~m}, 2 \mathrm{H}), 7.60(\mathrm{tt}, J=7.4,1.3 \mathrm{~Hz}, 1 \mathrm{H}), 7.52-7.48$ $(\mathrm{m}, 2 \mathrm{H}), 7.20-7.14(\mathrm{~m}, 2 \mathrm{H}) .{ }^{13} \mathbf{C} \mathbf{N M R}\left(\mathrm{CDCl}_{3}, 100 \mathrm{MHz}\right) \delta 195.2,166.6,164.1,137.5$, $133.8,133.7,132.7,132.6,132.4,129.8,128.3,115.5,115.3$. 
<smiles>O=C(c1ccccc1)c1ccc(C(=O)c2ccccc2)cc1</smiles>

1,4-dibenzoylbenzene (11) was prepared according to the general procedure but with 4 equiv $(0.6 \mathrm{mmol})$ of TMP, 10 equiv $(3.0 \mathrm{mmol})$ of acetone and 3 equiv $(0.90 \mathrm{mmol})$ of PhOTf. Purification was done by column chromatography. The fractions were collected using a gradient of 5\% to 8\% EtOAc in hexane to afford $\mathbf{1 1}$ as a white solid $(63.9 \mathrm{mg}$, $74 \%$ yield $)$. Characterization data matched those previously reported. ${ }^{6}{ }^{1} \mathbf{H} \mathbf{~ N M R}\left(\mathrm{CDCl}_{3}\right.$, $400 \mathrm{MHz}) \delta 7.90(\mathrm{~s}, 4 \mathrm{H}), 7.86-7.84(\mathrm{~m}, 4 \mathrm{H}), 7.66-7.61(\mathrm{~m}, 2 \mathrm{H}), 7.55-7.51(\mathrm{~m}, 4 \mathrm{H}) .{ }^{13} \mathbf{C}$ NMR $\left(\mathrm{CDCl}_{3}, 100 \mathrm{MHz}\right) \delta 196.0,140.6,136.9,133.0,130.1,129.7,128.5$.<smiles>O=C(c1ccccc1)c1ccc(-c2ccccc2)cc1</smiles>

[1,1'-biphenyl]4-yl(phenyl)methanone (12) was prepared according to the general procedure on $3.0 \mathrm{mmol}$ scale. Purification was done by column chromatography. The fractions were collected using a gradient of $2 \%$ to $6 \%$ EtOAc in hexane to afford $\mathbf{1 2}$ as a white solid $(69.3 \mathrm{mg}, 89 \%$ yield). Characterization data matched those previously reported. ${ }^{7}{ }^{1} \mathbf{H}$ NMR $\left(\mathrm{CDCl}_{3}, 400 \mathrm{MHz}\right) \delta$ 7.92-7.90 (m, 2H), 7.86-7.84 (m, 2H), 7.73$7.71(\mathrm{~m}, 2 \mathrm{H}), 7.68-7.66(\mathrm{~m}, 2 \mathrm{H}), 7.62(\mathrm{tt}, J=6.7,1.2 \mathrm{~Hz}, 1 \mathrm{H}), 7.50(\mathrm{q}, J=7.8 \mathrm{~Hz}, 4 \mathrm{H})$, $7.42(\mathrm{tt}, J=6.4,1.2 \mathrm{~Hz}, 1 \mathrm{H}) .{ }^{13} \mathbf{C} \mathbf{~ N M R}\left(\mathrm{CDCl}_{3}, 100 \mathrm{MHz}\right) \delta 196.3,145.2,140.0,137.7$, $136.2,132.4,130.7,130.0,128.9,128.3,128.2$.<smiles>O=C(c1ccccc1)c1ccc2ccccc2c1</smiles>

2-benzoylnaphthalene (13) was prepared according to the general procedure from benzylalcohol and naphthalen-2-yl trifluoromethanesulfonate. Purification was done by column chromatography. The fractions were collected using a gradient of $3 \%$ to $5 \%$ EtOAc in hexane to afford $\mathbf{1 3}$ as a white solid (52.2 $\mathrm{mg}, 75 \%$ yield). Characterization data matched those previously reported. ${ }^{5}{ }^{1} \mathbf{H} \mathbf{~ N M R}\left(\mathrm{CDCl}_{3}, 400 \mathrm{MHz}\right) \delta 8.28(\mathrm{~s}, 1 \mathrm{H})$, 7.96-7.91 (m, 4H), 7.89-7.86 (m, 2H), 7.65-7.60 (m, 2H), 7.58-7.51 (m, 3H). ${ }^{13} \mathbf{C ~ N M R}$ $\left(\mathrm{CDCl}_{3}, 100 \mathrm{MHz}\right) \delta 196.7,137.9,135.2,134.8,132.4,132.2,131.8,130.1,129.4,128.3$, 128.3, 128.3, 126.8, 125.8. 
<smiles>COc1ccc(C(=O)c2ccccc2)c2ccccc12</smiles>

4-methoxynaphthalen-1-yl) (phenyl)methanone (14) was prepared according to the general procedure. Purification was done by column chromatography. The fractions were collected using a gradient of 5\% to $9 \%$ EtOAc in hexane to afford 14 as a light oil (67.2 $\mathrm{mg}, 86 \%$ yield). Characterization data matched those previously reported. ${ }^{8}{ }^{1} \mathbf{H}$ NMR $\left(\mathrm{CDCl}_{3}, 400 \mathrm{MHz}\right) \delta 8.41-8.37(\mathrm{~m}, 2 \mathrm{H}), 8.87-7.85(\mathrm{~m}, 2 \mathrm{H}), 7.63-7.53(\mathrm{~m}, 4 \mathrm{H}), 7.50-7.45$ $(\mathrm{m}, 2 \mathrm{H}), 6.80(\mathrm{~d}, J=8.1 \mathrm{~Hz}, 1 \mathrm{H}), 4.08(\mathrm{~s}, 3 \mathrm{H}) .{ }^{13} \mathbf{C} \mathbf{N M R}\left(\mathrm{CDCl}_{3}, 100 \mathrm{MHz}\right) \delta 197.3$, $158.3,139.4,132.6,132.5,131.4,130.3,128.2$, 128.1, 128.0, 125.8, 125.7, 125.7, 122.2, $101.9,55.7$.<smiles>COc1ccc(C(=O)c2ccccc2)cc1</smiles>

4-methoxybenzophenone (15) was prepared according to the general procedure from benzylalcohol and 4-methoxyphenyl trifluoromethanesulfonate. 15 was obtained as a light oil (60.4 mg, 90\% yield). Purification and characterization data identical to compound 6.<smiles>O=C(c1ccccc1)c1ccc(C(F)(F)F)cc1</smiles>

4-(trifluoromethyl)benzophenone (16) was prepared according to the general procedure from benzylalcohol and 4-(trifluoromethyl)phenyl trifluoromethanesulfonate. 16 was obtained as a white solid (52.4 mg, 70\% yield). Purification and characterization data identical to compound $\mathbf{1 0 .}$<smiles>CC(=O)c1ccc(C(=O)c2ccccc2)cc1</smiles>

4-acetylbenzophenone (17) was prepared according to the general procedure. Purification was done by column chromatography. The fractions were collected using a 
gradient of $1 \%$ to $6 \%$ EtOAc in hexane to afford $\mathbf{1 7}$ as a white solid (591. $\mathrm{mg}, 88 \%$ yield). Characterization data matched those previously reported. ${ }^{4}{ }^{1} \mathbf{H}$ NMR $\left(\mathrm{CDCl}_{3}, 400\right.$ MHz) $\delta 8.06(\mathrm{dt}, J=8.2,1.6 \mathrm{~Hz}, 2 \mathrm{H}), 7.87(\mathrm{dt}, J=8.2,1.6 \mathrm{~Hz}, 2 \mathrm{H}), 7.82-7.79(\mathrm{~m}, 2 \mathrm{H})$, 7.63 (tt, $J=6.8,1.3 \mathrm{~Hz}, 1 \mathrm{H}), 7.53-7.49(\mathrm{~m}, 2 \mathrm{H}), 2.67$ (s, 3H). ${ }^{13} \mathrm{C} \mathbf{~ N M R}\left(\mathrm{CDCl}_{3}, 100\right.$ MHz) $\delta 197.5,195.9,141.3,139.5,136.9,132.9,130.0,130.0,128.4,128.1,26.8$.<smiles>Cc1cccc(C)c1C(=O)c1ccccc1</smiles>

(2,6-dimethylphenyl)(phenyl)methanone (18) was prepared according to the general procedure. Purification was done by column chromatography. The fractions were collected using a gradient of $1 \%$ to $3 \%$ EtOAc in hexane to afford $\mathbf{1 8}$ as a white solid (52.3 mg, 83\% yield). Characterization data matched those previously reported. ${ }^{9}{ }^{1} \mathbf{H}$ NMR $\left(\mathrm{CDCl}_{3}, 400 \mathrm{MHz}\right) \delta 7.83-7.81(\mathrm{~m}, 2 \mathrm{H}), 7.60(\mathrm{tt}, J=6.8,1.3 \mathrm{~Hz}, 1 \mathrm{H}), 7.46(\mathrm{t}, J=$ $8.2 \mathrm{~Hz}, 2 \mathrm{H}), 7.25(\mathrm{t}, J=7.5 \mathrm{~Hz}, 1 \mathrm{H}), 7.09$ (d, $J=7.5 \mathrm{~Hz}, 2 \mathrm{H}), 2.14(\mathrm{~s}, 6 \mathrm{H}) .{ }^{13} \mathbf{C}$ NMR $\left(\mathrm{CDCl}_{3}, 100 \mathrm{MHz}\right) \delta 200.4,139.6,137.0,134.1,133.6,129.4,128.8,128.7,127.5$, 19.3.<smiles>O=C(c1ccccc1)c1ccc(Cl)cc1</smiles>

4-chlorobenzophenone (19) was prepared according to the general procedure. Purification was done by column chromatography. The fractions were collected using a gradient of $0 \%$ to $10 \%$ EtOAc in hexane to afford 19 as a light oil $(46.7 \mathrm{mg}, 72 \%$ yield). Characterization data matched those previously reported. ${ }^{4}{ }^{\mathbf{1}} \mathbf{H} \mathbf{~ N M R}\left(\mathrm{CDCl}_{3}, 400 \mathrm{MHz}\right) \delta$ 7.82-7.75 (m, 4H), $7.61(\mathrm{tt}, J=6.8,1.3 \mathrm{~Hz}, 1 \mathrm{H}), 7.52-7.45(\mathrm{~m}, 4 \mathrm{H}) .{ }^{13} \mathbf{C} \mathbf{~ N M R}\left(\mathrm{CDCl}_{3}\right.$, $100 \mathrm{MHz}) \delta 195.5,138.9,137.2,135.8,132.6,131.4,129.9,128.6,128.4$.<smiles>CC(=O)Nc1ccc(C(=O)c2ccccc2)cc1</smiles>

N-(4-benzoylphenyl)acetamide (20) was prepared according to the general procedure. Purification was done by column chromatography. The fractions were collected using a gradient of $40 \%$ EtOAc to 60\% EtOAc in hexane with $1 \% \mathrm{Et}_{3} \mathrm{~N}$ to afford 20 as a white powder $\left(65 \mathrm{mg}, 91 \%\right.$ yield). Characterization data matched those previously reported. ${ }^{10}$ ${ }^{1} \mathbf{H}$ NMR $\left(\mathrm{CDCl}_{3}, 400 \mathrm{MHz}\right) \delta 8.33$ (br. s, $\left.1 \mathrm{H}\right), 7.79-7.73$ (m, 4H), 7.67-7.65 (m, 2H), 
7.59-7.55 (m, 1H), 7.48-7.44 (m, 2H), 2.19 (s, 3H). ${ }^{13} \mathbf{C}$ NMR $\left(\mathrm{CDCl}_{3}, 100 \mathrm{MHz}\right) \delta$ 196.0, 169.1, 142.3, 137.8, 132.8, 132.4, 131.6, 129.9, 128.3, 118.9, 24.7.

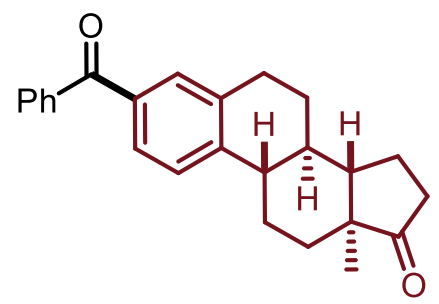

3-benzoylestra-1,3,5(10)-trien-17-one (21) was prepared according to the general procedure. Purification was done by column chromatography. The fractions were collected using a gradient of $20 \%$ to $30 \%$ EtOAc in hexane to afford $\mathbf{2 1}$ as a white solid (96.2 mg, 90\% yield). Characterization data matched those previously reported. ${ }^{4}{ }^{1} \mathbf{H}$ NMR $\left(\mathrm{CDCl}_{3}, 400 \mathrm{MHz}\right) \delta 7.82-7.79(\mathrm{~m}, 2 \mathrm{H}), 7.61-7.57(\mathrm{~m}, 3 \mathrm{H}), 7.50-7.46(\mathrm{~m}, 2 \mathrm{H})$, $7.40(\mathrm{~d}, J=7.9 \mathrm{~Hz}, 1 \mathrm{H}), 3.00-2.96(\mathrm{~m}, 2 \mathrm{H}), 2.57-2.46(\mathrm{~m}, 2 \mathrm{H}), 2.42-2.36(\mathrm{~m}, 1 \mathrm{H}), 2.22-$ 1.99 (m, 4H), 1.71-1.45 (m, 6H), 0.94 (s, 3H). ${ }^{13} \mathbf{C ~ N M R ~}\left(\mathrm{CDCl}_{3}, 100 \mathrm{MHz}\right) \delta$ 196.6, $144.8,137.9,136.7,135.1,132.2,130.6,129.9,128.2,127.7,125.2,50.5,47.9,44.7$, $37.9,35.8,31.5,29.3,26.3,25.6,21.6,13.8$.<smiles>O=C(CCc1cccnc1)c1ccccc1</smiles>

1-phenyl-3-(pyridin-3-yl)propan-1-one (22) was prepared according to the general procedure. Purification was done by column chromatography. The fractions were collected using a gradient of $30 \%$ to $50 \%$ acetone in hexane to afford $\mathbf{2 2}$ as a white solid (45.9 $\mathrm{mg}, 73 \%$ yield). Characterization data matched those previously reported. ${ }^{11}{ }^{1} \mathbf{H}$ NMR $\left(\mathrm{CDCl}_{3}, 400 \mathrm{MHz}\right) \delta 8.54(\mathrm{~d}, J=2.0 \mathrm{~Hz}, 1 \mathrm{H}), 8.46(\mathrm{dd}, J=4.8,1,5 \mathrm{~Hz}, 1 \mathrm{H}), 7.97-$ $7.94(\mathrm{~m}, 2 \mathrm{H}), 7.60-55(\mathrm{~m}, 2 \mathrm{H}), 7.48-7.44(\mathrm{~m}, 2 \mathrm{H}), 7.23-7.20(\mathrm{~m}, 1 \mathrm{H}), 3.33(\mathrm{t}, J=7.4 \mathrm{~Hz}$, $2 \mathrm{H}), 3.09(\mathrm{t}, J=7.5 \mathrm{~Hz}, 2 \mathrm{H}) .{ }^{13} \mathrm{C} \mathbf{~ N M R}\left(\mathrm{CDCl}_{3}, 100 \mathrm{MHz}\right) \delta 198.4,150.0,147.7,136.6$, 136.6, 136.0. 133.2, 128.6, 128.0, 123.3, 39.7, 27.1.<smiles>O=C(Cc1c[nH]c2ccccc12)c1ccccc1</smiles>

2-(1H-indol-3-yl)-1-phenylethanone (23) was prepared according to the general procedure. Purification was done by column chromatography. The fractions were collected using a gradient of $10 \%$ to $40 \%$ EtOAc in hexane to afford $\mathbf{2 3}$ as a pale red 
solid (51.1 mg, $88 \%$ yield). Characterization data matched those previously reported. ${ }^{12}$ ${ }^{1} \mathbf{H}$ NMR $\left(\mathrm{CDCl}_{3}, 400 \mathrm{MHz}\right) \delta 8.11$ (br s, $\left.1 \mathrm{H}, \mathrm{NH}\right), 8.09-8.07$ (m, 2H), 7.63 (d, $J=7.8$ $\mathrm{Hz}, 1 \mathrm{H}), 7.56(\mathrm{tt}, J=7.2,1.2 \mathrm{~Hz}, 1 \mathrm{H}), 7.48-7.44(\mathrm{~m}, 2 \mathrm{H}), 7.36(\mathrm{~d}, J=8.0 \mathrm{~Hz}, 1 \mathrm{H}), 7.22$ $(\mathrm{td}, J=7.0,1.2 \mathrm{~Hz}, 1 \mathrm{H}), 7.18-7.12(\mathrm{~m}, 2 \mathrm{H}), 4.43(\mathrm{~d}, J=0.8 \mathrm{~Hz}, 2 \mathrm{H}) .{ }^{13} \mathbf{C ~ N M R}\left(\mathrm{CDCl}_{3}\right.$, $100 \mathrm{MHz}) \delta 197.8,136.7,136.1,133.0,128.6,128.6,127.3,123.1,122.2$, 119.7, 118.7, 11.2, 109.0, 35.5.<smiles>O=C(c1ccccc1)c1ccco1</smiles>

2-furyl(phenyl)methanone (24) was prepared according to the general procedure at 140 ${ }^{\circ} \mathrm{C}$. Purification was done by column chromatography. The fractions were collected using a gradient of $5 \%$ to $12 \%$ EtOAc in hexane to afford 24 as a yellow oil $(41.9 \mathrm{mg}, 81 \%$ yield). Characterization data matched those previously reported. ${ }^{4}{ }^{1} \mathbf{H} \mathbf{~ N M R}\left(\mathrm{CDCl}_{3}, 400\right.$ MHz) $\delta 8.00-7.97(\mathrm{~m}, 2 \mathrm{H}), 7.72(\mathrm{dd}, J=1.7,0.8 \mathrm{~Hz}, 1 \mathrm{H}), 7.61(\mathrm{tt}, J=7.4,1.3 \mathrm{~Hz}, 1 \mathrm{H})$, 7.53-7.49 (m, 2H), $7.25(\mathrm{dd}, J=3.6,0.8 \mathrm{~Hz}, 1 \mathrm{H}), 6.61(\mathrm{dd}, J=3.6,1.7 \mathrm{~Hz}, 1 \mathrm{H}) .{ }^{13} \mathbf{C}$ NMR $\left(\mathrm{CDCl}_{3}, 100 \mathrm{MHz}\right) \delta 182.6,152.3,147.1,137.3,132.6,129.3,128.4,120.5,112.2$.<smiles>O=C(c1ccccc1)c1cccs1</smiles>

2-benzoylthiophene (25) was prepared according to the general procedure. Purification was done by column chromatography. The fractions were collected using a gradient of $0 \%$ to $3 \%$ EtOAc in hexane to afford 25 as a light oil (52.3 $\mathrm{mg}$, 93\% yield). Characterization data matched those previously reported. ${ }^{4} \mathbf{1} \mathbf{H} \mathbf{N M R}\left(\mathrm{CDCl}_{3}, 400 \mathrm{MHz}\right) \delta$ 7.89-7.86 (m, 2H), 7.73 (dd, $J=4.9,1.1 \mathrm{~Hz}, 1 \mathrm{H}), 7.66$ (dd, $J=3.8,1.1 \mathrm{~Hz}, 1 \mathrm{H}), 7.60$ (tt, $J=6.6,1.3 \mathrm{~Hz}, 1 \mathrm{H}), 7.53-7.49(\mathrm{~m}, 2 \mathrm{H}), 7.18-7.16(\mathrm{~m}, 2 \mathrm{H}) .{ }^{13} \mathbf{C} \mathbf{N M R}\left(\mathrm{CDCl}_{3}, 100 \mathrm{MHz}\right)$ $\delta 188.2,143.6,138.1,134.8,134.2,132.2,129.2,128.4,127.9$.<smiles>O=C(c1ccccc1)c1ccc2ncccc2c1</smiles>

phenyl(quinolin-6-yl)methanone (26) was prepared according to the general procedure. Purification was done by column chromatography. The fractions were collected using a gradient of $10 \%$ to $50 \%$ EtOAc in hexane to afford 26 as a light oil (52.5 mg, $75 \%$ yield). Characterization data matched those previously reported. ${ }^{13} \mathbf{1} \mathbf{H} \mathbf{~ N M R}\left(\mathrm{CDCl}_{3}, 400 \mathrm{MHz}\right)$ 
$\delta 9.04(\mathrm{dd}, J=4.2,1.7 \mathrm{~Hz}, 1 \mathrm{H}), 8.27-8.16(\mathrm{~m}, 4 \mathrm{H}), 7.88-7.86(\mathrm{~m}, 2 \mathrm{H}), 7.65(\mathrm{tt}, J=6.7$, $1.3 \mathrm{~Hz}, 1 \mathrm{H}), 7.56-7.49(\mathrm{~m}, 3 \mathrm{H}) .{ }^{13} \mathbf{C}$ NMR $\left(\mathrm{CDCl}_{3}, 100 \mathrm{MHz}\right) \delta 196.0,152.4,149.8$, $137.4,137.3,135.4,132.6,131.3,130.0,129.8,129.5,128.4,127.3,122.0$.<smiles>O=C(c1ccccc1)c1cccnc1</smiles>

phenyl(pyridine-3-yl)methanone (27) was prepared according to the general procedure. Purification was done by column chromatography. The fractions were collected using a gradient of $2 \%$ to $6 \%$ EtOAc in hexane to afford 27 as a yellow oil ( $44.6 \mathrm{mg}, 81 \%$ yield). Characterization data matched those previously reported. ${ }^{4}{ }^{1} \mathbf{H} \mathbf{~ N M R}\left(\mathrm{CDCl}_{3}, 400 \mathrm{MHz}\right) \delta$ $9.01(\mathrm{~s}, 1 \mathrm{H}), 8.83(\mathrm{~s}, 1 \mathrm{H}), 8.15(\mathrm{~d}, J=7.4 \mathrm{~Hz}, 1 \mathrm{H}), 7.83(\mathrm{~d}, J=7.2 \mathrm{~Hz}, 2 \mathrm{H}), 7.65(\mathrm{t}, J=$ 7.4 Hz, $1 \mathrm{H}), 7.55-7.48(\mathrm{~m}, 3 \mathrm{H}) .{ }^{13} \mathbf{C}$ NMR $\left(\mathrm{CDCl}_{3}, 100 \mathrm{MHz}\right) \delta 194.9,152.8,151.0$, 137.1, 136.7, 133.2, 130.0, 128.6, 123.3.<smiles>O=C(c1ccccc1)c1cccc2nccnc12</smiles>

phenyl(quinoxalin-6-yl)methanone (28) was prepared according to the general procedure. Purification was done by column chromatography using $50 \%$ EtOAc in Hexane. After column a mixture of product and quinoline was obtained. The mixture was further purified by water/toluene extraction (3X). Combined organic layers, dried with $\mathrm{Na}_{2} \mathrm{SO}_{4}$, concentrated under reduced pressure and dried via schlenk while heating to obtain 28 as dark red solid (29.9 mg, 43\% yield). Characterization data matched those previously reported. ${ }^{14} \mathbf{1} \mathbf{H}$ NMR $\left(\mathrm{CDCl}_{3}, 400 \mathrm{MHz}\right) \delta 8.87(\mathrm{~d}, J=1.7 \mathrm{~Hz}, 1 \mathrm{H}), 8.77(\mathrm{~d}, J$ $=1.8 \mathrm{~Hz}, 1 \mathrm{H}), 8.26(\mathrm{dd}, J=7.8,2.1 \mathrm{~Hz}, 1 \mathrm{H}), 7.89-7.80(\mathrm{~m}, 4 \mathrm{H}), 7.60-7.55(\mathrm{~m}, 1 \mathrm{H}), 7.42$ $(\mathrm{t}, J=7.6 \mathrm{~Hz}, 2 \mathrm{H}) .{ }^{13} \mathrm{C}$ NMR $\left(\mathrm{CDCl}_{3}, 100 \mathrm{MHz}\right) \delta 196.6,145.6,145.2,142.6,141.2$, $139.5,137.6,133.6,131.5,130.2,129.4,129.0,128.5$.<smiles>O=C(c1ccccc1)c1ccc2ncsc2c1</smiles>

benzo[d]thiazol-6-yl(phenyl)methanone (29) was prepared according to the general procedure. Purification was done by column chromatography. The fractions were collected using using gradient of $0 \%$ to $20 \%$ EtOAc in hexane to afford 29 as white 
powder (46.0 mg, 64\% yield). ${ }^{1} \mathbf{H}$ NMR $\left(\mathrm{CDCl}_{3}, 400 \mathrm{MHz}\right) \delta 9.28(\mathrm{~s}, 1 \mathrm{H}), 8.49(\mathrm{~s}, 1 \mathrm{H})$, $8.25(\mathrm{~d}, J=8.4 \mathrm{~Hz}, 1 \mathrm{H}), 8.01(\mathrm{~d}, J=7.0 \mathrm{~Hz}, 1 \mathrm{H}), 7.90-7.79(\mathrm{~m}, 2 \mathrm{H}), 7.68-7.60(\mathrm{~m}, 1 \mathrm{H})$, 7.58-7.50 (m, 2H). ${ }^{13} \mathbf{C}$ NMR $\left(\mathrm{CDCl}_{3}, 100 \mathrm{MHz}\right) \delta 195.8,157.4,156.1,137.6,134.9$, 132.6, 130.0, 128.4, 127.9, 124.7, 123.4, 115.1. Accurate mass (EI): Theoretical: 239.0399. Found: 239.0422. Spectral Accuracy: 98.2\%. FT-IR: $v\left(\mathrm{~cm}^{-1}\right)$ 3053, 1648, 1595, 1546, 1432, 1401, 1308, 1254, 1154, 1051, 950, 873, 857, 747, 700.<smiles>O=C(CCCCCCl)c1ccccc1</smiles>

6-chloro-1-phenylhexan-1-one (30) was prepared according to the general procedure. Purification was done by column chromatography. The fractions were collected using a gradient of $1 \%$ to 5\% EtOAc in hexane to afford $\mathbf{3 0}$ as a light oil (45.3 $\mathrm{mg}, 72 \%$ yield). Characterization data matched those previously reported. ${ }^{15}{ }^{1} \mathbf{H} \mathbf{~ N M R}\left(\mathrm{CDCl}_{3}, 400 \mathrm{MHz}\right)$ $\delta$ 7.98-7.95 (m, 2H), $7.57(\mathrm{tt}, J=6.6,1.3 \mathrm{~Hz}, 2 \mathrm{H}), 7.49-7.45(\mathrm{~m}, 2 \mathrm{H}), 3.57(\mathrm{t}, J=6.7 \mathrm{~Hz}$, $2 \mathrm{H}), 3.00(\mathrm{t}, J=7.2 \mathrm{~Hz}, 2 \mathrm{H}), 1.88-1.75(\mathrm{~m}, 4 \mathrm{H}), 1.59-1.51(\mathrm{~m}, 2 \mathrm{H}) .{ }^{13} \mathbf{C} \mathbf{N M R}\left(\mathrm{CDCl}_{3}\right.$, $100 \mathrm{MHz}) \delta 199.9,136.9,133.0,128.6,128.0,44.8,38.3,32.4,26.6,23.4$.<smiles>O=C(CCc1ccccc1)c1ccccc1</smiles>

1,3-diphenylpropan-1-one (31) was prepared according to the general procedure. Purification was done by column chromatography. The fractions were collected using a gradient of $1 \%$ to $3 \%$ EtOAc in hexane to afford $\mathbf{3 1}$ as a light oil (47.3 $\mathrm{mg}, 75 \%$ yield). Characterization data matched those previously reported (keto-enol tautomerisation visible by NMR). ${ }^{5}{ }^{1} \mathbf{H}$ NMR $\left(\mathrm{CDCl}_{3}, 400 \mathrm{MHz}\right) \delta 7.99-7.96(\mathrm{~m}, 2 \mathrm{H}), 7.57$ (tt, $J=7.3,1.3$ $\mathrm{Hz}, 1 \mathrm{H}), 7.48-7.44(\mathrm{~m}, 2 \mathrm{H}), 7.33-7.26(\mathrm{~m}, 4 \mathrm{H}), 7.24-7.19(\mathrm{~m}, 1 \mathrm{H}), 3.32(\mathrm{t}, J=8.2 \mathrm{~Hz}$, $2 \mathrm{H}), 3.08(\mathrm{t}, J=7.3 \mathrm{~Hz}, 2 \mathrm{H}) .{ }^{13} \mathbf{C} \mathbf{~ N M R}\left(\mathrm{CDCl}_{3}, 100 \mathrm{MHz}\right) \delta 199.2,141.3,136.8,133.0$, 128.6, 128.5, 128.4, 128.0, 126.1, 40.4, 30.1.<smiles>O=C(c1ccccc1)C1CCCCC1</smiles>

benzoylcyclohexane (32) was prepared according to the general procedure. Purification was done by column chromatography. The fractions were collected using a gradient of $1 \%$ to $5 \%$ EtOAc in hexane to afford $\mathbf{3 2}$ as a light oil (34.2 $\mathrm{mg}, 78 \%$ yield). Characterization data matched those previously reported. ${ }^{16}{ }^{1} \mathbf{H} \mathbf{~ N M R}\left(\mathrm{CDCl}_{3}, 400 \mathrm{MHz}\right)$ $\delta$ 7.97-7.94 (m, 2H), 7.55 (tt, $J=6.4,1.2 \mathrm{~Hz}, 1 \mathrm{H}), 7.48-7.45(\mathrm{~m}, 2 \mathrm{H}), 3.27(\mathrm{tt}, J=11.1$, 
$3.0 \mathrm{~Hz}, 1 \mathrm{H}), 1.92-1.83(\mathrm{~m}, 4 \mathrm{H}), 1.77-1.71(\mathrm{~m}, 1 \mathrm{H}), 1.59-1.22(\mathrm{~m}, 5 \mathrm{H}) .{ }^{13} \mathbf{C ~ N M R}\left(\mathrm{CDCl}_{3}\right.$, $100 \mathrm{MHz}) \delta 203.9,136.4,132.7,128.6,128.2,45.6,29.4,25.9,25.8$.<smiles>O=C(c1ccccc1)C1CC1</smiles>

benzoylcyclopropane (33) was prepared according to the general procedure at $140{ }^{\circ} \mathrm{C}$. Purification was done by column chromatography. The fractions were collected using a gradient of $1 \%$ to $4 \%$ EtOAc in hexane to afford $\mathbf{3 3}$ as a yellow oil (29.8 $\mathrm{mg}, 68 \%$ yield). Characterization data matched those previously reported. ${ }^{17} \mathbf{1}_{\mathbf{H}} \mathbf{N M R}\left(\mathrm{CDCl}_{3}, 400 \mathrm{MHz}\right)$ $\delta$ 8.04-8.02 (m, 2H), $7.57(\mathrm{tt}, J=6.5,1.3 \mathrm{~Hz}, 1 \mathrm{H}), 7.50-7.46(\mathrm{~m}, 2 \mathrm{H}), 2.72-2.66(\mathrm{~m}, 1 \mathrm{H})$, 1.27-1.24 (m, 2H), 1.07-1.03 (m, 2H) ${ }^{13} \mathbf{C}$ NMR $\left(\mathrm{CDCl}_{3}, 100 \mathrm{MHz}\right) \delta 200.6,138.0$, 132.7, 128.4, 128.0, 17.1, 11.6.<smiles>COc1ccc2cc(C(C)C(=O)c3ccccc3)ccc2c1</smiles>

2-(6-methoxynaphthalen-2-yl)-1-phenylpropan-1-one (34) was prepared according to the general procedure. Purification was done by column chromatography. The fractions were collected using a gradient of 5\% to 7\% DCM in hexane to afford 34 as a white solid (59.1 mg, 68\% yield). ${ }^{1} \mathbf{H}$ NMR $\left(\mathrm{CDCl}_{3}, 400 \mathrm{MHz}\right) \delta 8.02-7.98(\mathrm{~m}, 2 \mathrm{H}), 7.71-7.66(\mathrm{~m}$, $3 \mathrm{H}), 7.45$ (tt, $J=6.4,1.3 \mathrm{~Hz}, 1 \mathrm{H}) .{ }^{13} \mathbf{C} \mathbf{~ N M R}\left(\mathrm{CDCl}_{3}, 100 \mathrm{MHz}\right) \delta 200.4,157.6,136.6$, $136.5,133.5,132.7,129.2,128.8,128.4,127.6,126.4,126.2,119.0,105.5,55.3,47.8$, 19.5. Accurate mass (EI): Theoretical: 290.1307. Found: 290.1301. Spectral Accuracy: 99.3\%. FT-IR: $v\left(\mathrm{~cm}^{-1}\right)$ 2924, 1674, 1632, 1602, 1483, 1449, 1392, 1328, 1268, 1202, $1168,1118,1027,1002,963,922,895,859,819,756,711,660,564,535$. Mp: 107-110 ${ }^{\circ} \mathrm{C}$.<smiles>O=C(c1ccccc1)C1CCC1</smiles>

benzoylcyclobutane (35) was prepared according to the general procedure. Purification was done by column chromatography. The fractions were collected using a gradient of $1 \%$ to $3 \%$ EtOAc in hexane to afford 35 as a yellow oil (40.5 mg, 84\% yield). Characterization data matched those previously reported. ${ }^{18} \mathbf{1}_{\mathbf{H}} \mathbf{N M R}\left(\mathrm{CDCl}_{3}, 400 \mathrm{MHz}\right)$ $\delta$ 7.92-7.89 (m, 2H), $7.54(\mathrm{tt}, J=6.5,1.3 \mathrm{~Hz}, 1 \mathrm{H}), 7.47-7.42(\mathrm{~m}, 2 \mathrm{H}), 4.05-3.96(\mathrm{~m}, 1 \mathrm{H})$, 
2.47-2.38 (m, 2H), 2.34-2.26 (m, 2H), 2.15-2.03 (m, 1H), 1.96-1.86 (m, 1H). ${ }^{13} \mathbf{C}$ NMR $\left(\mathrm{CDCl}_{3}, 100 \mathrm{MHz}\right) \delta 200.9,135.6,132.8,128.5,128.3,42.1,25.0,18.1$.

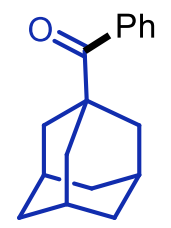

1-adamantyl(phenyl)methanone (36) was prepared according to the general procedure with $\mathrm{Ni}(\operatorname{cod})_{2}(20 \mathrm{~mol} \%)$ and Triphos $(24 \mathrm{~mol} \%)$ at $140{ }^{\circ} \mathrm{C}$. Purification was done by column chromatography. The fractions were collected using a gradient of $1 \%$ to $3 \%$ EtOAc in hexane to afford $\mathbf{3 6}$ as a white solid ( $36.1 \mathrm{mg}, 53 \%$ yield). Characterization data matched those previously reported. ${ }^{19}{ }^{1} \mathbf{H}$ NMR $\left(\mathrm{CDCl}_{3}, 400 \mathrm{MHz}\right) \delta 7.56-7.53(\mathrm{~m}$, 2H), 7.46-7.36 (m, 3H), 2.08 (m, 3H), 2.02-2.01 (m, 6H), 1.79-1.71 (m, 6H). ${ }^{13} \mathbf{C}$ NMR $\left(\mathrm{CDCl}_{3}, 100 \mathrm{MHz}\right) \delta 210.2,139.6,130.1,127.9,46.9,39.1,36.5,28.1$.<smiles>CC[C@H](C)C(=O)c1ccccc1</smiles>

(S)-2-methyl-1-phenylbutan-1-one (37) was prepared according to the general procedure. After cooling the reaction mixture to room temperature, aqueous hydrogen peroxide solution $(30 \mathrm{w} \%, 100 \mu \mathrm{L})$ and distilled $\mathrm{H}_{2} \mathrm{O}(1 \mathrm{~mL})$ were added to oxidize Triphos. The organic layer was separated and the aqueous layer was further extracted with EtOAc. The organic layers were combined and concentrated under reduced pressure. The fractions were collected using a gradient of $0 \%$ to $8 \% \mathrm{Et}_{2} \mathrm{O}$ in pentane to afford 37 as a colourless oil $(15.5 \mathrm{mg}, 32 \%$ yield, $91 \%$ ee). Characterization data matched those previously reported. ${ }^{20}{ }^{1} \mathbf{H}$ NMR $\left(\mathrm{CDCl}_{3}, 400 \mathrm{MHz}\right) \delta 7.97-7.93(\mathrm{~m}, 2 \mathrm{H}), 7.58-7.52(\mathrm{~m}$, $1 \mathrm{H}), 7.50-7.42(\mathrm{~m}, 2 \mathrm{H}), 3.40(\mathrm{~h}, J=6.8 \mathrm{~Hz}, 1 \mathrm{H}), 1.84(\mathrm{dqd}, J=13.8,7.4,6.5 \mathrm{~Hz}, 1 \mathrm{H})$, $1.58-1.38(\mathrm{~m}, 1 \mathrm{H}), 1.19(\mathrm{~d}, J=6.9 \mathrm{~Hz}, 3 \mathrm{H}), 0.93(\mathrm{~d}, J=7.4 \mathrm{~Hz}, 3 \mathrm{H}) .{ }^{13} \mathbf{C} \mathbf{~ N M R}$ $\left(\mathrm{CDCl}_{3}, 100 \mathrm{MHz}\right) \delta 204.5,136.9,132.8,128.6,128.2,42.1,26.7,16.8,11.8$. Enantiomeric ratio was measured by cHPLC (OB-H column, 10\% IPA in hexane, 0.5 $\mathrm{mL} / \mathrm{min}, 25{ }^{\circ} \mathrm{C}$, detection at $254 \mathrm{~nm}$ ). Traces of chiral and racemic sample are shown in section 5 . 
<smiles>COC(=O)[C@H](Cc1ccc(C(=O)c2ccccc2)cc1)NC(=O)c1ccccc1</smiles>

methyl (S)-3-(4-benzoylphenyl)-2-((tert-butoxycarbonyl)amino)propanoate (38) was prepared according to the general procedure on $0.2 \mathrm{mmol}$ scale. Purification was done by column chromatography. The fractions were collected using $20 \%$ EtOAc in hexane to afford 38 as a colourless oil $(46.2 \mathrm{mg}, 60 \%, 94 \%$ ee). Characterization data matched those previously reported. ${ }^{21} \mathbf{1} \mathbf{H}$ NMR $\left(\mathrm{CDCl}_{3}, 400 \mathrm{MHz}\right) \delta 7.79-7.74(\mathrm{~m}, 4 \mathrm{H}), 7.61-7.57$ $(\mathrm{m}, 1 \mathrm{H}), 7.50-7.46(\mathrm{~m}, 2 \mathrm{H}), 7.25(\mathrm{~d}, J=6.8 \mathrm{~Hz}, 2 \mathrm{H}), 5.03(\mathrm{~d}, J=7.5 \mathrm{~Hz}, 1 \mathrm{H}), 4.67-4.62$ $(\mathrm{m}, 1 \mathrm{H}), 3.74(\mathrm{~s}, 3 \mathrm{H}), 3.26-3.09(\mathrm{~m}, 2 \mathrm{H}), 1.42(\mathrm{~s}, 9 \mathrm{H}) .{ }^{13} \mathbf{C ~} \mathbf{N M R}\left(\mathrm{CDCl}_{3}, 100 \mathrm{MHz}\right) \delta$ 196.3, 172.0, 155.0, 141.1, 137.6, 136.3, 132.4, 130.4, 130.0, 129.3, 128.3, 80.1, 54.2, $52.4,38.4,28.3$. Enantiomeric ratio was measured by SFC (IA chiral column; 2.0 $\mathrm{mL} / \mathrm{min}$, supercritical fluid chromatography $10 \% \mathrm{MeOH} / \mathrm{CO}_{2}, 200 \mathrm{bar}, 60{ }^{\circ} \mathrm{C}$, detection at $254 \mathrm{~nm}$. Traces of chiral and racemic sample are shown in section 5.<smiles>CCCCCCCCCCCCCCC1=C(C)C(=O)C(OC)=C(OC)C1=O</smiles>

\section{2,3-dimethoxy-5-(10-(4-methoxyphenyl)-10-oxodecyl)-6-methylcyclohexa-2,5-diene-}

1,4-dione (39) was prepared according to the general procedure on $0.2 \mathrm{mmol}$ scale at 140 ${ }^{\circ} \mathrm{C}$. Purification was done by column chromatography. The fractions were collected using $20 \%$ EtOAc in hexane with $1 \% \mathrm{Et}_{3} \mathrm{~N}$ to afford 39 as an orange oil (55.2 $\mathrm{mg}, 62 \%$ yield). ${ }^{1} \mathbf{H}$ NMR (400 MHz, CDCl3) $\delta 7.94(\mathrm{~d}, J=8.8 \mathrm{~Hz}, 2 \mathrm{H}), 6.93(\mathrm{~d}, J=8.8 \mathrm{~Hz}, 2 \mathrm{H}), 3.98$ (s, $6 \mathrm{H}), 3.87(\mathrm{~s}, 3 \mathrm{H}), 2.90(\mathrm{t}, J=7.4 \mathrm{~Hz}, 2 \mathrm{H}), 2.46-2.42(\mathrm{~m}, 2 \mathrm{H}), 2.00(\mathrm{~s}, 3 \mathrm{H}), 1.73-1.69(\mathrm{~m}$, 2H), 1.33 (br. s, $12 \mathrm{H}) .{ }^{13} \mathbf{C ~ N M R}\left(\mathrm{CDCl}_{3}, 100 \mathrm{MHz}\right) \delta$ 199.3, 184.8, 184.2, 163.2, 144.4, 143.1, 138.6, 130.3, 130.2, 113.7, 61.1, 55.5, 38.3, 29.8, 29.4, 29.3, 28.7, 26.4, 24.6, 11.9. Accurate mass (ESI): Theoretical for $\mathrm{C}_{26} \mathrm{H}_{34} \mathrm{O}_{6} \mathrm{Na}_{1}[\mathrm{M}+\mathrm{Na}]^{+}:$465.2253. Found: 465.2261. FT-IR: $v\left(\mathrm{~cm}^{-1}\right)$ 2926, 2852, 1648, 1604, 1510, 1455, 1364, 1258, 1204, 1166, 1070, 1027, 835, 741, 604 . 
<smiles>Cc1ccc(C(=O)c2ccc(C(C)(C)CC(C)(C)C)cc2Cc2ccc(Cl)cc2Cl)cc1</smiles>

(2-(2,4-dichlorobenzyl)-4-(2,4,4-trimethylpentan-2-yl)phenyl)(p-tolyl)methanone

(40) was prepared according to the general procedure. Purification was done by column chromatography. The fractions were collected using gradient $0 \%$ to $10 \%$ EtOAc in hexane to afford $\mathbf{4 0}$ as a colourless oil $(87.0 \mathrm{mg}, 62 \%$ yield $) .{ }^{1} \mathbf{H}$ NMR $\left(\mathrm{CDCl}_{3}, 400\right.$ $\mathrm{MHz}) \delta 7.64(\mathrm{~d}, J=8.2 \mathrm{~Hz}, 2 \mathrm{H}), 7.31-7.29(\mathrm{~m}, 3 \mathrm{H}), 7.23-7.20(\mathrm{~m}, 3 \mathrm{H}), 7.05(\mathrm{dd}, J=8.3$, $2.2 \mathrm{~Hz}, 1 \mathrm{H}), 6.94(\mathrm{~d}, J=8.3 \mathrm{~Hz}, 1 \mathrm{H}), 4.14(\mathrm{~s}, 2 \mathrm{H}), 2.42$ (s, 3H), 1.71 (s, 2H), 1.34 (s, $6 \mathrm{H}), 0.70(\mathrm{~s}, 9 \mathrm{H}) .{ }^{13} \mathbf{C ~ N M R}\left(\mathrm{CDCl}_{3}, 100 \mathrm{MHz}\right) \delta 197.9,153.0,143.9,137.6,137.4$, $135.9,135.4,134.7,132.4,132.0,130.4,129.0,129.0,129.0,126.8,123.6,56.8,38.8$, 36.3, 32.3, 31.8, 31.4, 21.7. Accurate mass (EI): Theoretical: 466.1830. Found: 466.1825. Spectral Accuracy: 98.8\%. FT-IR: $v\left(\mathrm{~cm}^{-1}\right)$ 2953, 1738, 1657, 1604, 1468, 1398, 1272, 1212, 1164, 1103, 1046, 939, 832, 781, 732, 688, 603.<smiles>CC1CCN(CCCC(=O)c2ccc(F)cc2)CC1</smiles>

1-(4-fluorophenyl)-4-(4-methylpiperidin-1-yl)butan-1-one (41) (Melperone) was prepared according to the general procedure. Purification was done by column chromatography. The fractions were collected using 50\% EtOAc in hexane with $5 \% \mathrm{Et}_{3} \mathrm{~N}$ to afford 41 (54.2 $\mathrm{mg}, 69 \%$ yield). Characterization data matched those previously reported. ${ }^{22}{ }^{1} \mathbf{H}$ NMR $\left(\mathrm{CDCl}_{3}, 400 \mathrm{MHz}\right) \delta 8.02-7.98(\mathrm{~m}, 2 \mathrm{H}), 7.14-7.10(\mathrm{~m}, 2 \mathrm{H}), 2.96(\mathrm{t}$, $J=7.1 \mathrm{~Hz}, 2 \mathrm{H}), 2.87-2.84(\mathrm{~m}, 2 \mathrm{H}), 2.37(\mathrm{t}, J=7.2 \mathrm{~Hz}, 2 \mathrm{H}), 1.95-1.88(\mathrm{~m}, 4 \mathrm{H}), 1.60-1.57$ $(\mathrm{m}, 2 \mathrm{H}), 1.36-1.30(\mathrm{~m}, 1 \mathrm{H}), 1.18-1.15(\mathrm{~m}, 2 \mathrm{H}), 0.89(\mathrm{~d}, J=6.5 \mathrm{~Hz}, 3 \mathrm{H}) .{ }^{13} \mathbf{C} \mathbf{~ N M R}$ $\left(\mathrm{CDCl}_{3}, 100 \mathrm{MHz}\right) \delta 198.6,165.6,133.6,130.7,128.3,115.5,58.0,53.9,36.4,34.2$, 30.8, 21.9. ${ }^{19} \mathbf{F ~ N M R ~}\left(\mathrm{CDCl}_{3}, 400 \mathrm{MHz}\right) \delta-105.9$. 
<smiles>CC1CCN(CCCC(=O)c2cccc(F)c2)CC1</smiles>

1-(3-fluorophenyl)-4-(4-methylpiperidin-1-yl)butan-1-one (42) was prepared according to the general procedure. Purification was done by column chromatography. The fractions were collected using 45\% EtOAc in hexane with $5 \% \mathrm{Et}_{3} \mathrm{~N}$ to afford 42 (52.4 mg, 66\% yield). Characterization data matched those previously reported. ${ }^{22}{ }^{\mathbf{1}} \mathbf{H}$ NMR $\left(\mathrm{CDCl}_{3}, 400 \mathrm{MHz}\right) \delta$ 7.76-7.74 (m, 1H), 7.67-7.64 (m, 1H), 7.46-7.40 (m, 1H), 7.25-7.22 (m, 1H), $2.96(\mathrm{t}, J=7.1 \mathrm{~Hz}, 2 \mathrm{H}), 2.86-2.83(\mathrm{~m}, 2 \mathrm{H}), 2.39-2.35(\mathrm{~m}, 2 \mathrm{H}), 1.96-$ $1.87(\mathrm{~m}, 4 \mathrm{H}), 1.60-1.56(\mathrm{~m}, 2 \mathrm{H}), 1.36-1.30(\mathrm{~m}, 1 \mathrm{H}), 1.22-1.13(\mathrm{~m}, 2 \mathrm{H}), 0.89$ (d, J = 6.5 $\mathrm{Hz}, 3 \mathrm{H}) .{ }^{13} \mathbf{C ~ N M R}\left(\mathrm{CDCl}_{3}, 100 \mathrm{MHz}\right) \delta 198.9,162.9,139.3,133.0130 .0,128.3,123.8$, $119.8,114.9,58.0,53.9,36.6,34.2,30.8,21.9 .{ }^{19} \mathbf{F ~ N M R}\left(\mathrm{CDCl}_{3}, 400 \mathrm{MHz}\right) \delta-112.1$.<smiles>CC1CCN(CCCC(=O)c2ccccc2F)CC1</smiles>

1-(3-fluorophenyl)-4-(4-methylpiperidin-1-yl)butan-1-one (43) was prepared according to the general procedure. Purification was done by column chromatography. The fractions were collected using using $45 \%$ EtOAc in hexane with $5 \% \mathrm{Et}_{3} \mathrm{~N}$ to afford $43\left(60.1 \mathrm{mg}, 76 \%\right.$ yield). Characterization data matched those previously reported. ${ }^{22}{ }^{1} \mathbf{H}$ NMR $\left(\mathrm{CDCl}_{3}, 400 \mathrm{MHz}\right) \delta 7.85(\mathrm{td}, J=7.6,1.8 \mathrm{~Hz}, 1 \mathrm{H}), 7.52-7.47(\mathrm{~m}, 1 \mathrm{H}), 7.26-7.19$ $(\mathrm{m}, 1 \mathrm{H}), 7.12(\mathrm{dd}, J=11.2,8.4 \mathrm{~Hz}, 1 \mathrm{H}), 2.98(\mathrm{td}, J=7.1,2.8 \mathrm{~Hz}, 2 \mathrm{H}), 2.89-2.86(\mathrm{~m}$, 2H), 2.39-2.35 (m, 2H), 1.96-1.88 (m, 4H), 1.60-1.57 (m, 2H), 1.29-1.16 (m, 3H), 0.89 $(\mathrm{d}, J=6.4 \mathrm{~Hz}, 3 \mathrm{H}) .{ }^{13} \mathrm{C} \mathbf{N M R}\left(\mathrm{CDCl}_{3}, 100 \mathrm{MHz}\right) \delta 198.5,161.8,134.2,130.7,124.4$, $124.3,116.6,58.2,53.9,41.5,34.2,30.9,22.0,21.7 .{ }^{19} \mathbf{F} \mathbf{N M R}\left(\mathrm{CDCl}_{3}, 400 \mathrm{MHz}\right) \delta-$ 109.6.<smiles>O=C(CCCN1CCC(O)(c2ccc(Cl)cc2)CC1)c1ccc(F)cc1</smiles>

4-(4-(4-chlorophenyl)-4-hydroxypiperidin-1-yl)-1-(4-fluorophenyl)butan-1-one (44) (Haloperidol) was prepared according to the general procedure. Purification was done by 
column chromatography. The fractions were collected using using gradient of $30 \%$ to $50 \%$ EtOAc in hexane with 5\% $\mathrm{Et}_{3} \mathrm{~N}$ to afford $\mathbf{4 4}(41.3 \mathrm{mg}$, 37\% yield). Characterization data matched those previously reported. ${ }^{23}{ }^{1} \mathbf{H}$ NMR $\left(\mathrm{CDCl}_{3}, 400 \mathrm{MHz}\right) \delta 8.03-8.00(\mathrm{~m}$, $2 \mathrm{H}), 7.38(\mathrm{~d}, J=8.6 \mathrm{~Hz}, 2 \mathrm{H}), 7.30(\mathrm{~d}, J=8.6 \mathrm{~Hz}, 2 \mathrm{H}), 7.16-7.11(\mathrm{~m}, 2 \mathrm{H}), 2.98(\mathrm{t}, J=7.0$ $\mathrm{Hz}, 2 \mathrm{H}), 2.81-2.78(\mathrm{~m}, 2 \mathrm{H}), 2.50-2.42(\mathrm{~m}, 4 \mathrm{H}), 2.03-1.96(\mathrm{~m}, 4 \mathrm{H}), 1.69-1.66(\mathrm{~m}, 2 \mathrm{H})$. ${ }^{13} \mathrm{C}$ NMR $\left(\mathrm{CDCl}_{3}, 100 \mathrm{MHz}\right) \delta 198.4,166.9,164.4,146.9,133.7,133.7,132.8,130.7$, 130.6, 128.4, 126.1, 115.7, 115.5, 71.1, 57.8, 49.3, 38.4, 36.2, 21.9. ${ }^{19} \mathbf{F}$ NMR $\left(\mathrm{CDCl}_{3}\right.$, $400 \mathrm{MHz}) \delta-105.7$. 


\section{Mechanistic studies}

\section{Identifying stoichiometric oxidants:}

As illustrated in Table S2 (entry 1 vs entry 24), the reaction of benzyl alcohol with phenyltriflate was found to be most efficient with acetone as an oxidant. However, 2decanone was found to give acceptable yields and allowed the quantification of reduced 2-decanol after reaction (Table S4). Organotriflate $\mathbf{4 6}$ was used to enable detection and quantification of the reduced biphenyl side-product.

Table S4. Comparison of oxidants.

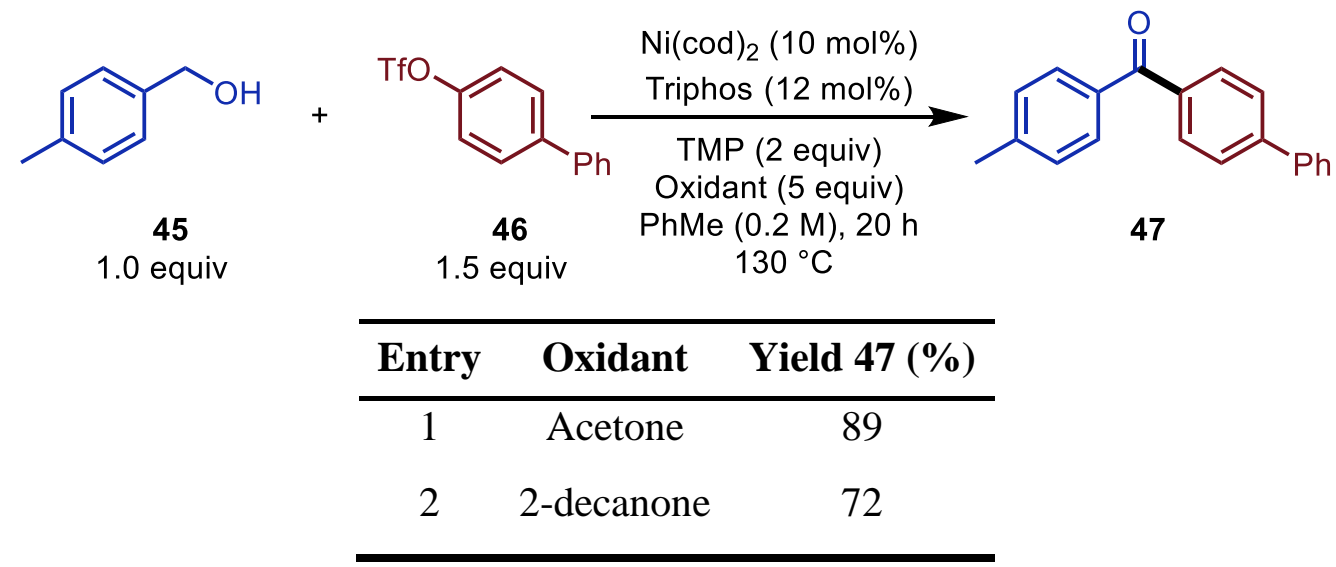

The reaction described in Scheme 3a was set up according to the general procedure (0.1 mmol scale; Scheme S7). The reaction mixture was cooled to room temperature after 20 h. 1,3,5-Trimethoxybenzene was added as an internal standard $(0.5 \mathrm{~mL}, 0.5 \mathrm{M})$ prior to filtering the reaction over a short pad of silica gel, washing with EtOAc. The filtrate was concentrated under reduced pressure and analyzed by ${ }^{1} \mathrm{H}$ NMR and by GC-FID using a 5 point calibration curve to determine the amount of product, biphenyl and 2-decanol. The experiment was performed in duplicate. The same experiment was repeated with acetone as the hydrogen acceptor. Despite giving a 16\% improved yield of ketone 47, a similar quantity of biphenyl 48a was observed. This suggests that acetone is a more effective hydrogen acceptor than 2-decanone, but we unfortunately could not directly quantify the isopropanol side-product. 


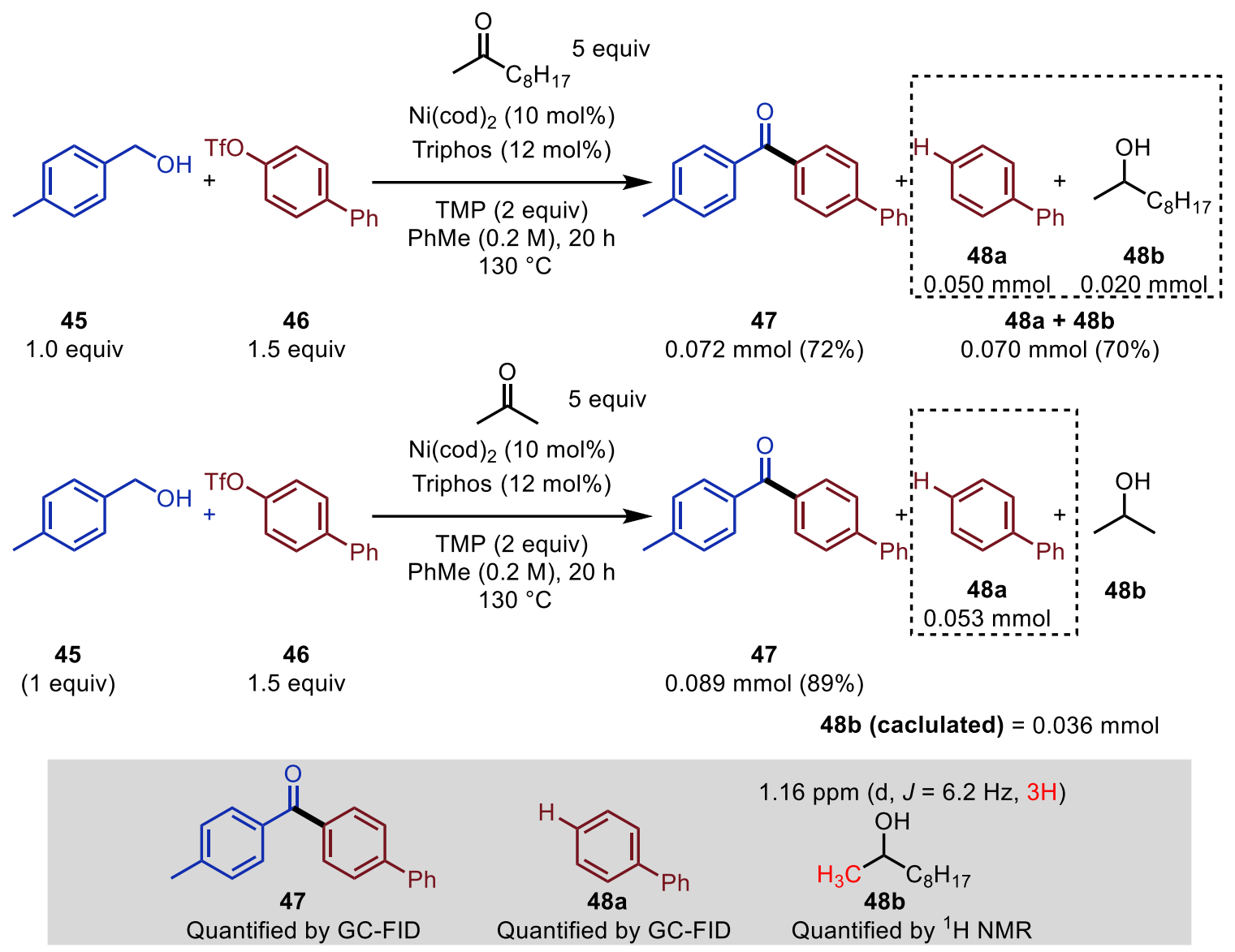

Scheme S7. Identifying and quantifying stoichiometric oxidants. Signal used for ${ }^{1} \mathrm{H}$ NMR quantification versus 1,3,5-Trimethoxybenzene internal standard shown in red. GC-FID quantification based on 5 point calibration curve. Values reported are the average of two runs.

\section{Deuterium incorporation:}

The following experiment with deuterated benzyl alcohol $\boldsymbol{d} \mathbf{- 4 5}$ was conducted to track deuterium incorporation (Scheme S8). Based on the proposed mechanism, we expect deuterium to incorporate in the reduced organotriflate by-product $\boldsymbol{d - 4 8 a}$ and in the reduced ketone by-product $\boldsymbol{d} \mathbf{- 4 8 b}$. Since excess acetone was the only highly effective ketone additive we know of, the 'ideal' experiment was modified to use a heavier but less effective ketone hydrogen acceptor in order to isolate the corresponding alcohol.

The reaction was performed under standard conditions with PMP instead of TMP to avoid exchange of the free $\mathrm{N}-\mathrm{H}$. The reaction described in Scheme S8 was set up 
according to the general procedure $(0.6 \mathrm{mmol}$ scale $)$. The reaction mixture was cooled to room temperature after $20 \mathrm{~h}$. The crude reaction mixture purified by column chromatography.

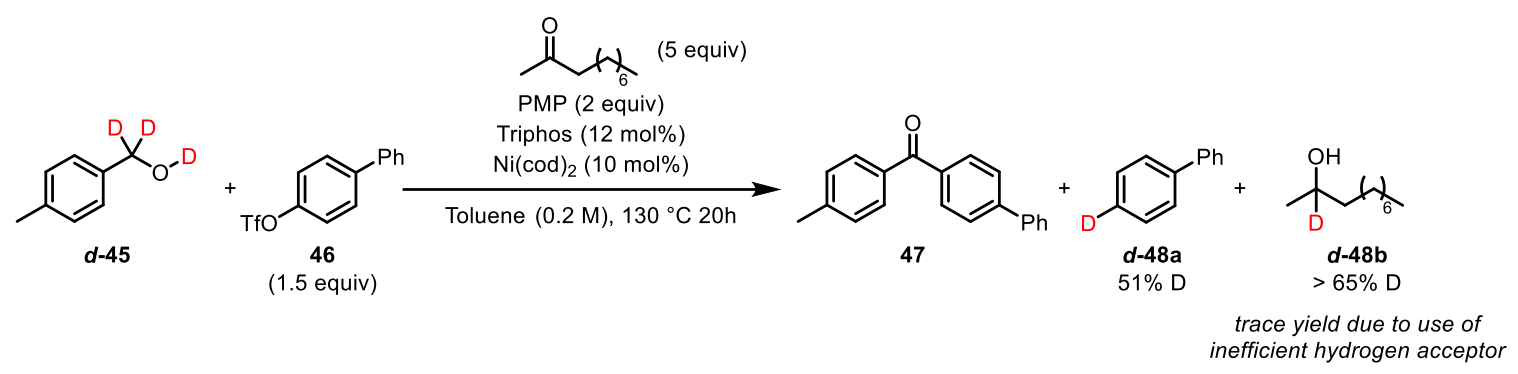

Scheme S8. Deuterium incorporation experiment.

A substantial incorporation of deuterium on the organotriflate is observed, though not $100 \%$. We suspect the $\alpha$-protons of the excess 2-decanone may be participating in exchange reactions.

Since 2-decanone is a relatively poor hydrogen acceptor that is used in excess (See Table S4), isolation of the pure reduced alcohol couldn't be achieved - contamination with the excess 2-decanone was prominent. While integration of the 2-decanol $\alpha$-proton could not be achieved with baseline resolution, a minimum $\%$ deuterium incorporation of $65 \%$ was detected.

\section{Observation \& importance of aldehyde intermediate:}

The reaction described in Scheme $3 b$ was performed as follows. A stock solution of $\mathrm{Ni}(\operatorname{cod})_{2}$, Triphos, Acetone, TMP, triflate 46, and alcohol 49 in Toluene was prepared according to the general procedure. From the stock solution 6 vials were filled $(0.1 \mathrm{mmol}$ of 3-phenyl-1-propanol/vial), capped and removed from the glove box, and placed in an oil bath pre-heated to $130{ }^{\circ} \mathrm{C}$ (starting point). After appropriate time the vial was removed from the oil bath and cooled under a flow of pressurized air. 1,3,5trimethoxybenzene was added as an internal standard $(0.5 \mathrm{~mL}, 0.5 \mathrm{M})$ prior to filtering the reaction over a short pad of silica gel, washing with EtOAc. The filtrate was concentrated under reduced pressure and analyzed by ${ }^{1} \mathrm{H}$ NMR to determine the amount of 3-phenyl-1-propanol, 3-phenyl-1-propanal and product (Scheme S9 and Table S5). 


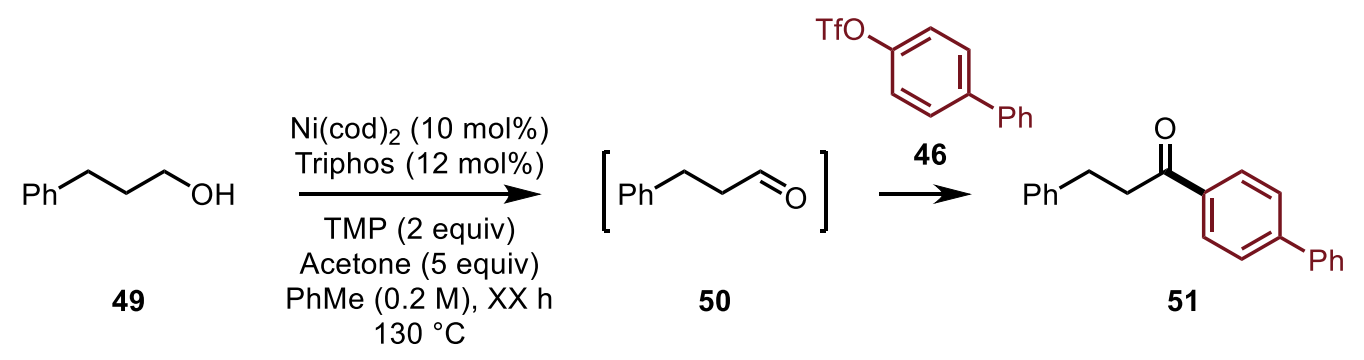

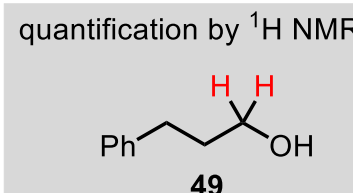

$3.69 \mathrm{ppm}(\mathrm{t}, \mathrm{J}=6.4 \mathrm{~Hz}, 2 \mathrm{H})$

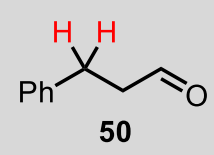

$2.97 \mathrm{ppm}(\mathrm{t}, J=7.6 \mathrm{~Hz}, 2 \mathrm{H})$

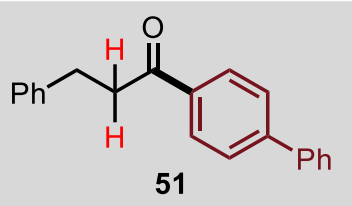

3.35 ppm (t, $J=7.7 \mathrm{~Hz}, 2 \mathrm{H})$

Scheme S9. Observation and importance of aldehyde intermediate. Signal used for ${ }^{1} \mathrm{H}$ NMR quantification versus 1,3,5-Trimethoxybenzene internal standard shown in red.

Table S5 and corresponding graph. Amount of 3-phenyl-1-propanol, 3-phenyl-1propanal and product determined by ${ }^{1} \mathrm{H}$ NMR with 1,3,5-trimethoxybenzene as an internal standard. Average value of 2 runs. Data used for plot Scheme $3 \mathrm{~b}$ in manuscript.

\begin{tabular}{cccc}
\hline Time (h) & $\begin{array}{c}\text { Product } \\
(\mathbf{M})\end{array}$ & $\begin{array}{c}\text { Aldehyde } \\
(\mathbf{M})\end{array}$ & Alcohol (M) \\
\hline 0 & 0.000 & 0.000 & 0.200 \\
0.25 & 0.017 & 0.019 & 0.179 \\
0.50 & 0.028 & 0.017 & 0.160 \\
1 & 0.046 & 0.018 & 0.141 \\
3 & 0.086 & 0.035 & 0.082 \\
5 & 0.117 & 0.030 & 0.049 \\
20 & 0.170 & 0.009 & 0.005 \\
\hline
\end{tabular}




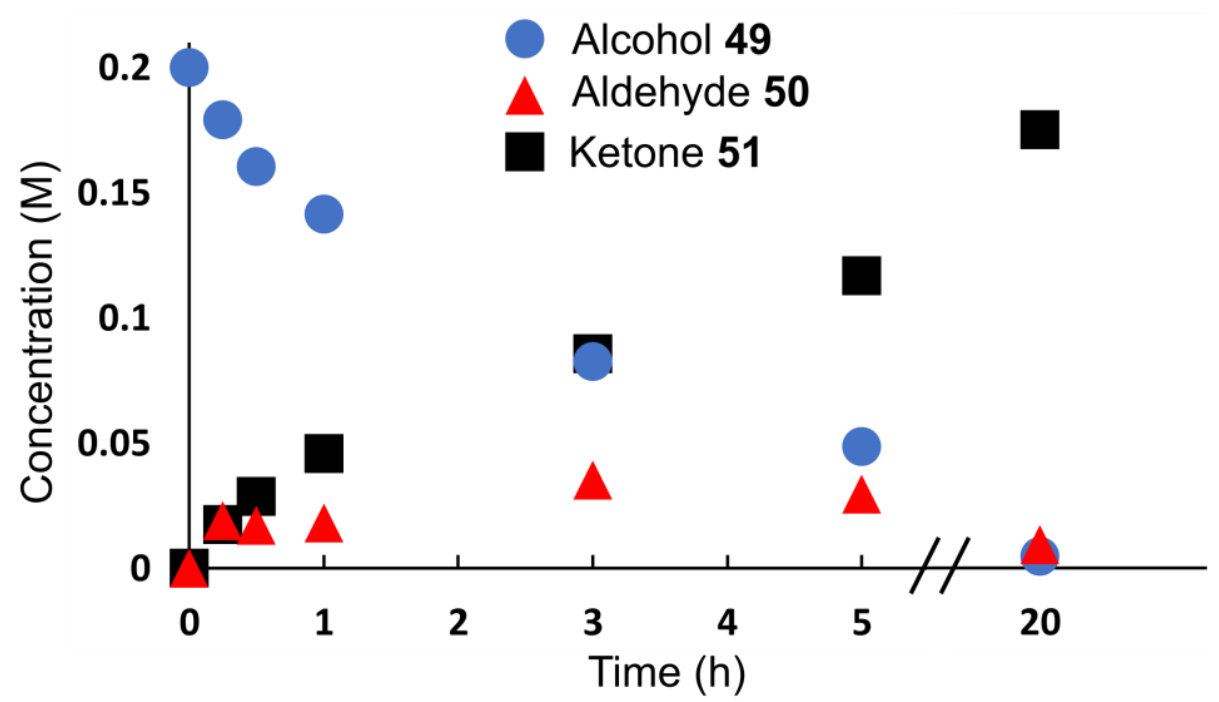

4. Observation of aldehyde dimerization side products: In our previous work on the use of aldehydes as reactive coupling partners for Ni-catalyzed coupling, use of aliphatic aldehydes was particularly problematic. ${ }^{4}$ These side-products were notably absent in the oxidative coupling with benzyl alcohols described herein. To illustrate this point, ketone 31 was prepared by several different methods (Scheme S10). Coupling of phenyltriflate with 3-Phenylpropionaldehyde $\mathbf{5 0}$ using any of the three optimized procedures developed led to product 31 being formed in 32 to $44 \%$ yield. In these reactions, substantial quantities of dimerization products $\mathbf{5 1}$ and $\mathbf{5 2}$ were observed, accounting up to $52 \%$ of the mass balance of the aldehyde starting material. In contrast, no dimerization products were observed when using alcohol 49 under oxidative coupling conditions, enabling a $78 \%$ yield of $\mathbf{3 1}$ to be obtained. It is likely that these conditions are efficient because aldehyde $\mathbf{5 0}$ is generated and consumed in situ at similar rates, preventing accumulation. Since the dimerization reaction is expected to be second order, keeping the concentration of aldehyde low in solution should slow down these side-reactions more than it slows down the productive pathway, resulting in improved selectivity.

The reactions described in Scheme S10 were set up according to the general procedure. After stirring for 20 hours, the reaction vial was removed from the oil bath and cooled down to room temperature. 1,3,5-trimethoxybenzene was added as an internal standard $(0.5 \mathrm{~mL}, 0.5 \mathrm{M})$ prior to filtering the reaction over a short pad of silica gel, washing with EtOAc. The filtrate was concentrated under reduced pressure and analyzed by ${ }^{1} \mathrm{H} \mathrm{NMR}$ 
to determine the amount of desired product, remaining starting material and Tishchenko and aldol side products.
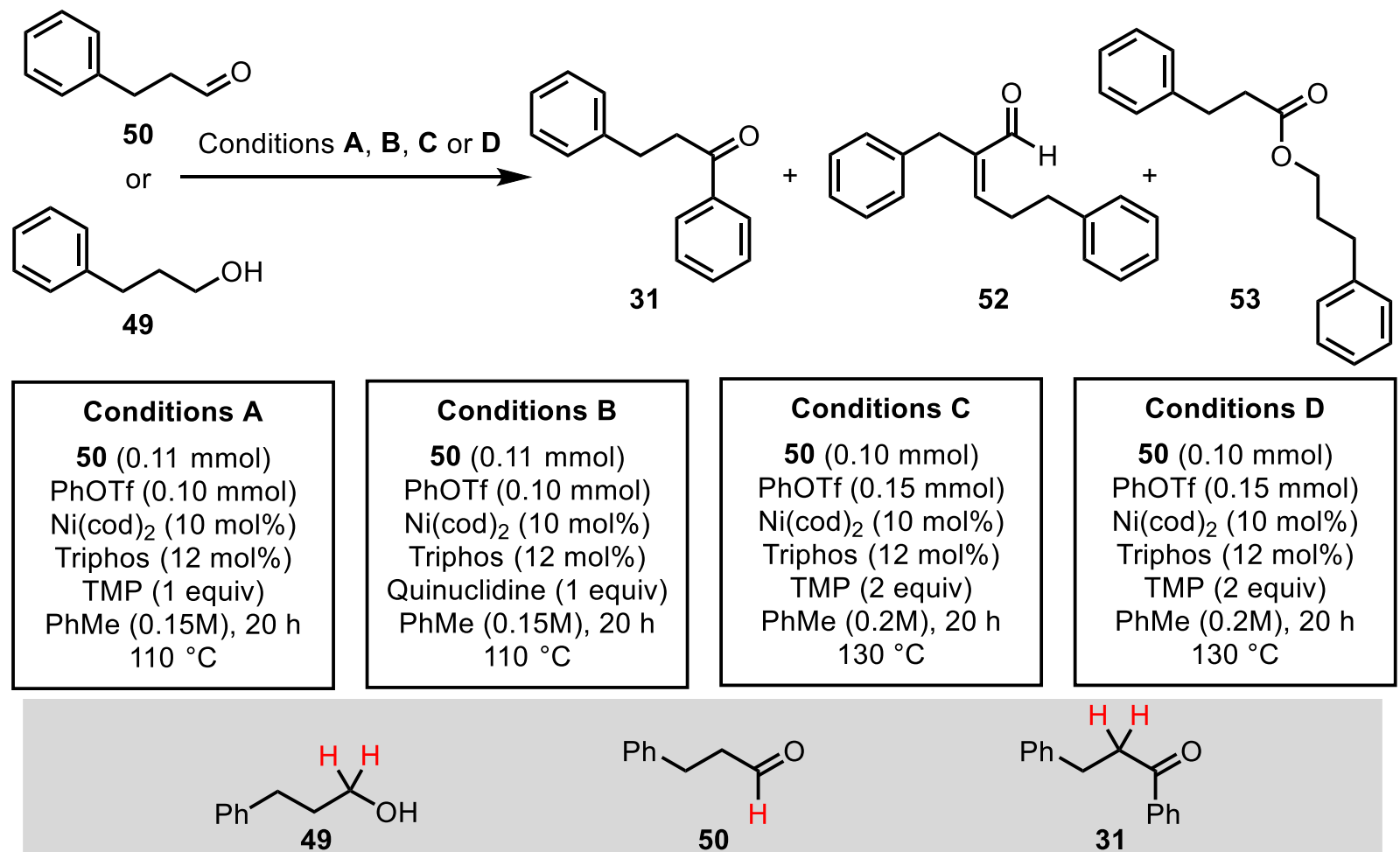

Conditions C

$50(0.10 \mathrm{mmol})$ PhOTf $(0.15 \mathrm{mmol})$ $\mathrm{Ni}(\mathrm{cod}))_{2}(10 \mathrm{~mol} \%)$ Triphos (12 mol\%) TMP (2 equiv) PhMe (0.2M), $20 \mathrm{~h}$ $130{ }^{\circ} \mathrm{C}$
Conditions D

$50(0.10 \mathrm{mmol})$ PhOTf $(0.15 \mathrm{mmol})$ $\mathrm{Ni}(\mathrm{cod})_{2}(10 \mathrm{~mol} \%)$ Triphos (12 mol\%)

TMP ( 2 equiv) PhMe (0.2M), $20 \mathrm{~h}$ $130{ }^{\circ} \mathrm{C}$

$3.69 \mathrm{ppm}(\mathrm{t}, \mathrm{J}=6.4 \mathrm{~Hz}, 2 \mathrm{H})$

$9.83 \mathrm{ppm}(\mathrm{s}, 1 \mathrm{H})$

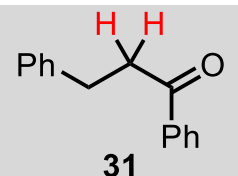<smiles></smiles>

53<smiles>O=C/C(=C\CCc1ccccc1)C(c1ccccc1)c1ccccc1</smiles>

52

2.64 ppm $(\mathrm{m}, 4 \mathrm{H})$

Scheme S10. Aldehyde starting material side product identification.

Table S6. Aldehyde starting material side product quantification.

\begin{tabular}{cccc}
\hline Conditions & $\begin{array}{c}\text { mmol 31 } \\
\text { (NMR yield) }\end{array}$ & $\begin{array}{c}\text { mmol 52 } \\
\text { (NMR yield) }\end{array}$ & $\begin{array}{c}\text { mmol 53 }^{\mathbf{a}} \\
\text { (NMR yield) }\end{array}$ \\
\hline A & $0.032(32 \%)$ & $0.008(15 \%)$ & $0.006(11 \%)$ \\
B & $0.032(32 \%)$ & $0.026(47 \%)$ & $0.003(5 \%)$ \\
C & $0.044(44 \%)$ & $0.006(11 \%)$ & $0.003(5 \%)$ \\
D & $0.078(78 \%)$ & \multicolumn{2}{c}{ Not observed } \\
a\% yield based on maximum possible quantity of dimerization products formed $(0.055$ mmol). \\
\hline
\end{tabular}




\section{5. cHPLC and Supercritical Fluid Chromatography data}

Chiral HPLC data for compound $\mathbf{3 7}$ and racemic reference are given below. Data was measured by cHPLC (OB-H column, $10 \%$ IPA in hexane, $0.5 \mathrm{~mL} / \mathrm{min}, 25^{\circ} \mathrm{C}$, detection at $254 \mathrm{~nm}$ ).

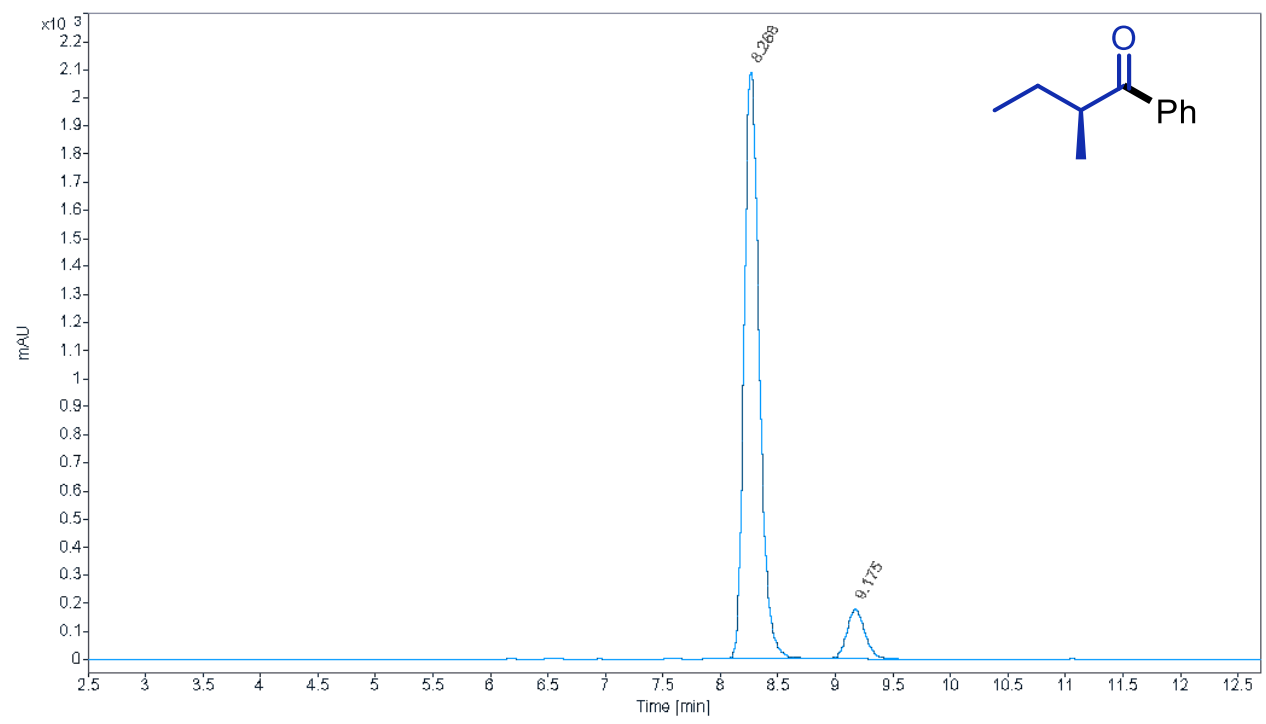

\begin{tabular}{|c|c|c|c|c|c|c|}
\hline & & & & & & \\
\hline RT [min] & Compound Name & Area & Height & Area $\%$ & RespFactor & Amount (ng/ul) \\
\hline 8.268 & & 19190.473 & 2088.184 & 90.9 & & 0.000 \\
\hline 9.175 & & 1931.408 & 176.565 & 9.1 & & 0.000 \\
\hline
\end{tabular}

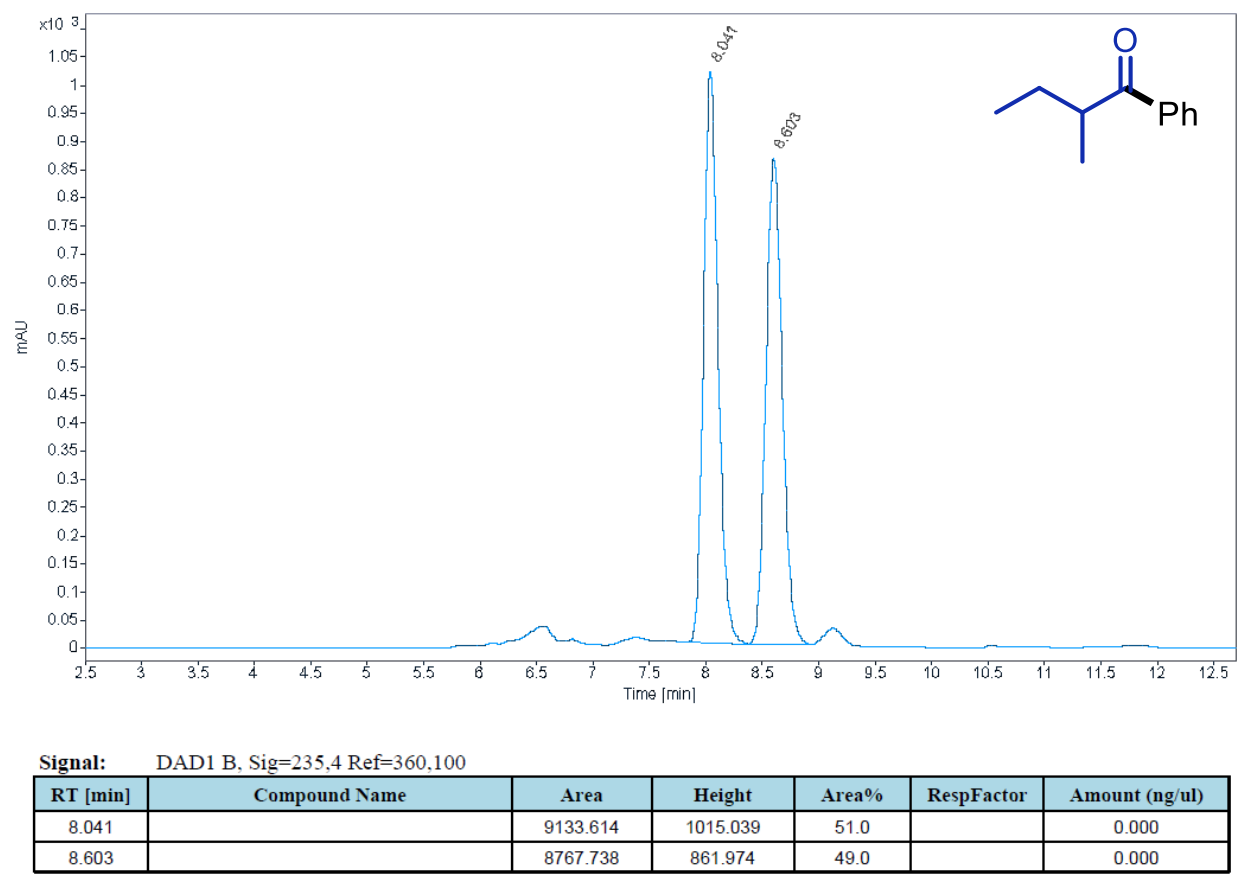


Chiral SFC data for compound $\mathbf{3 8}$ and racemic reference are given below. The racemic substrate was obtained according to the procedure reported for compound $\mathbf{3 8}$ with achiral tyrosine triflate. Data was measured by SFC (IA chiral column; $2.0 \mathrm{~mL} / \mathrm{min}$, supercritical fluid chromatography $10 \% \mathrm{MeOH} / \mathrm{CO}_{2}, 200 \mathrm{bar}, 60^{\circ} \mathrm{C}$, detection at $254 \mathrm{~nm}$ ).
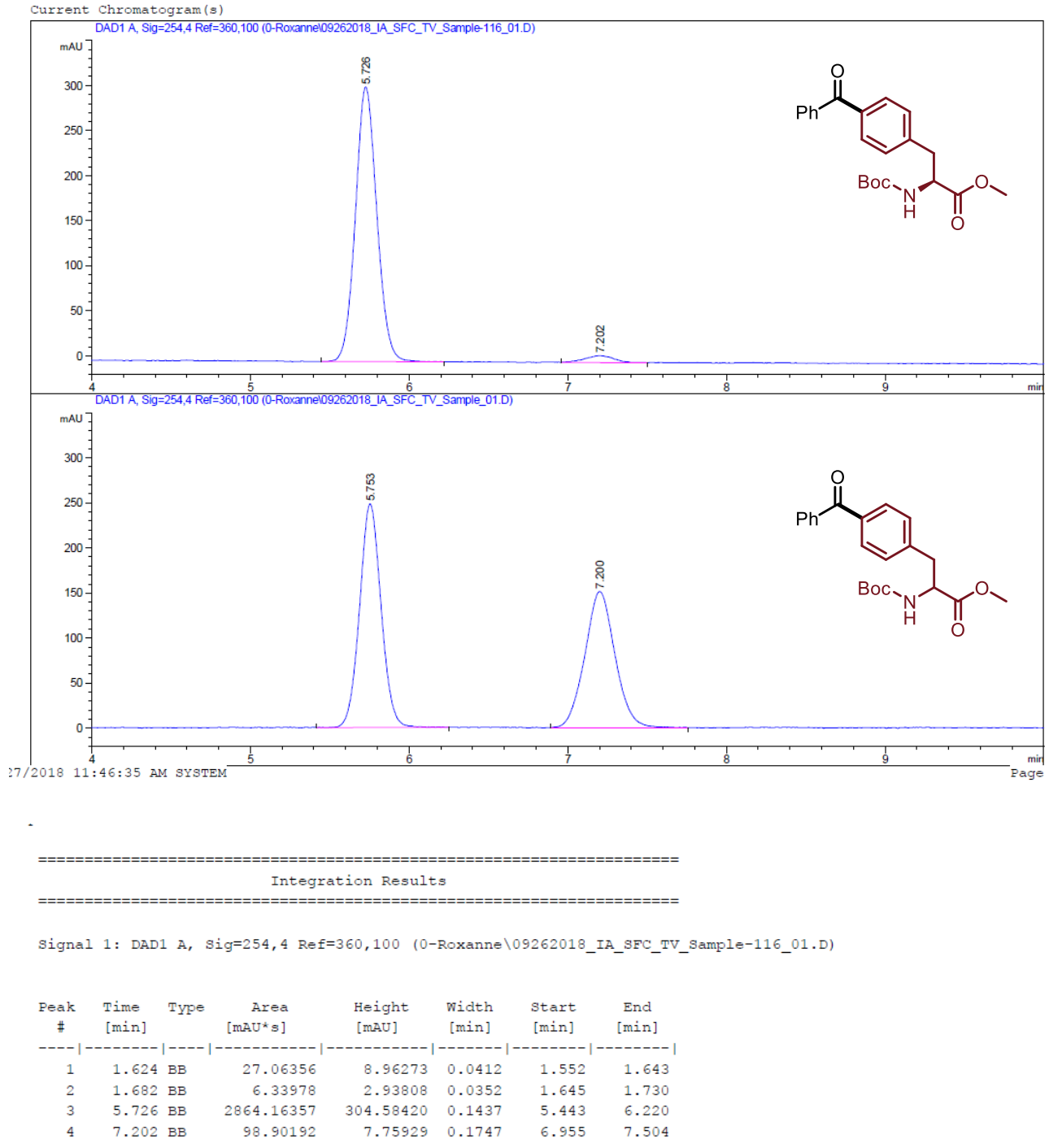

Signa1 2: DAD1 A, Sig=254,4 Ref=360,100 (0-Roxannel09262018_IA_SFC_TV_Sample_01.D)

\begin{tabular}{|c|c|c|c|c|c|c|c|}
\hline $\begin{array}{c}\text { Peak } \\
\#\end{array}$ & $\begin{array}{l}\text { Time } \\
{[\mathrm{min}]}\end{array}$ & TYpe & $\begin{array}{r}\text { Area } \\
{\left[\mathrm{mAU} U^{*} \mathrm{~s}\right]}\end{array}$ & $\begin{array}{l}\text { Height } \\
\text { [mAU] }\end{array}$ & $\begin{array}{l}\text { Width } \\
{[\mathrm{min}]}\end{array}$ & $\begin{array}{l}\text { Start } \\
{[\mathrm{min}]}\end{array}$ & $\begin{array}{l}\text { End } \\
{[\mathrm{min}]}\end{array}$ \\
\hline 1 & 1,623 & $B B$ & 30.45420 & 8 & 0.0485 & 544 & ---1 \\
\hline 2 & 1.669 & $\mathrm{BB}$ & $\begin{array}{r}5.26816\end{array}$ & 2.93591 & 0.0269 & 1.650 & 1.713 \\
\hline 3 & 5.753 & $\mathrm{BB}$ & 2342.02856 & 247.94701 & 0.1442 & 5.416 & 6.252 \\
\hline 4 & 7.200 & $\mathrm{BB}$ & 2006.79382 & 151.18445 & 0.2009 & 6.889 & 7.756 \\
\hline
\end{tabular}




\section{NMR Spectra}

2-(2,4-dichlorobenzyl)-4-(2,4,4-trimethylpentan-2-yl)phenyl trifluoromethanesulfonate (S1). $\mathrm{CDCl}_{3}, 400 \mathrm{MHz}$ :
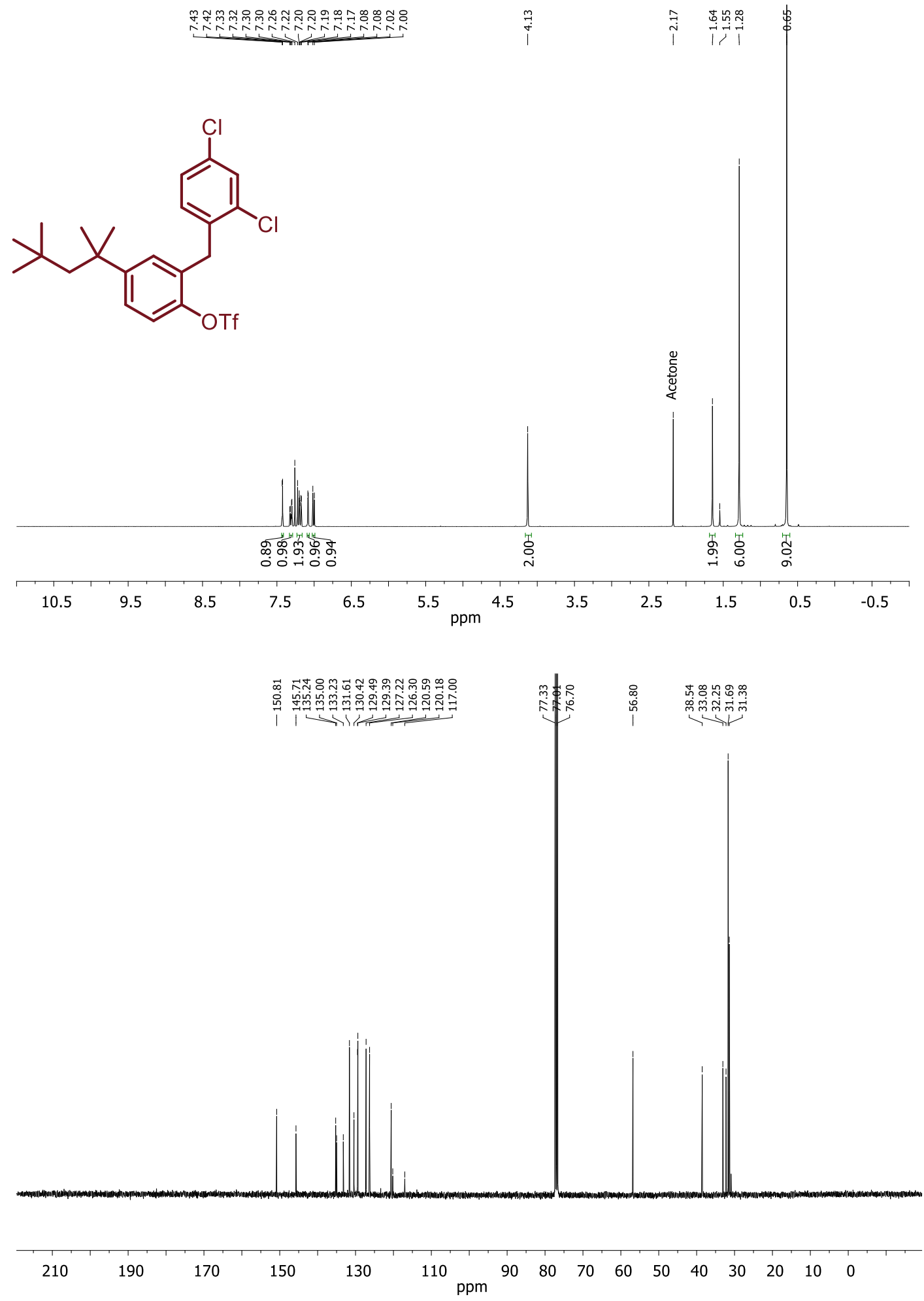


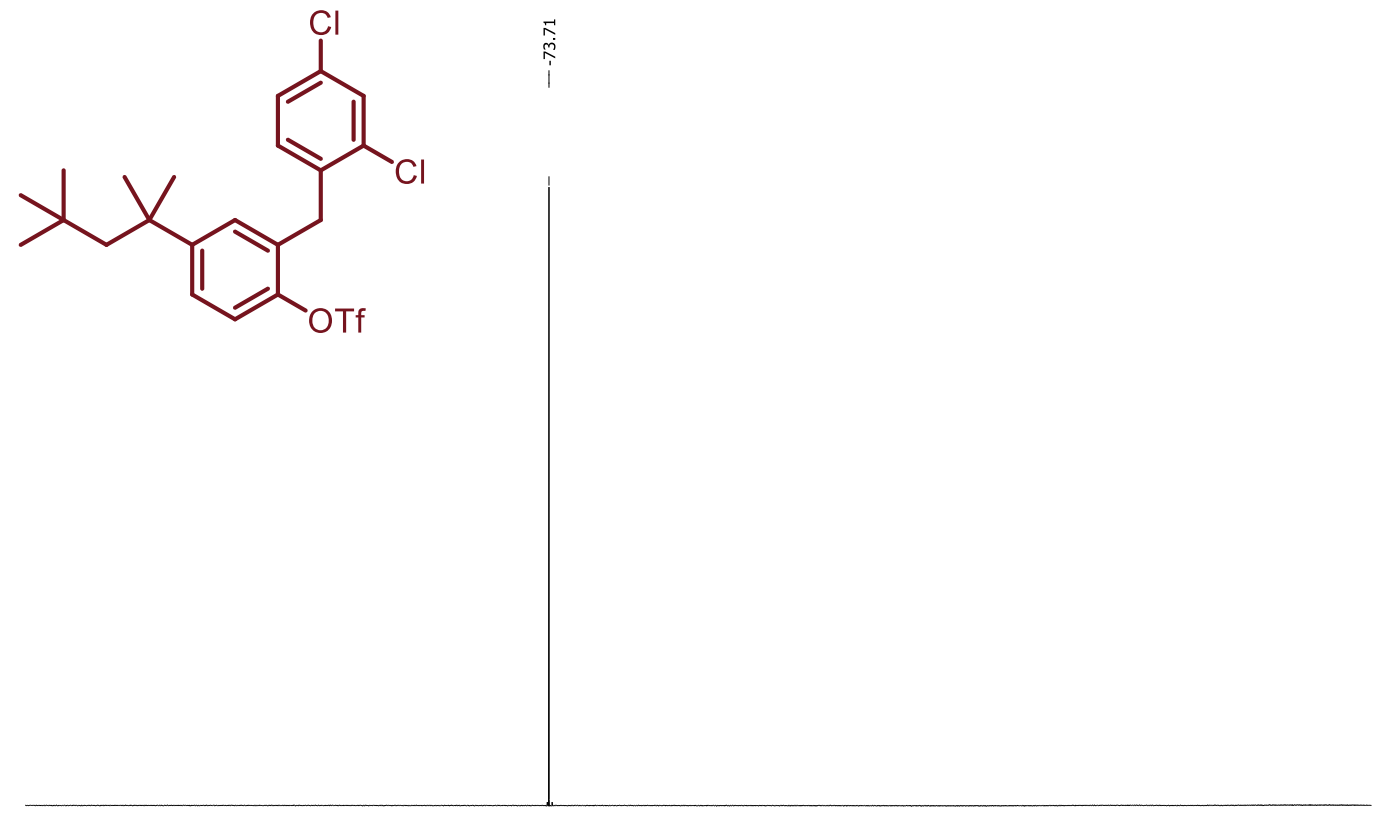

\begin{tabular}{|c|c|c|c|c|c|c|c|c|c|c|c|}
\hline 10 & 0 & -20 & -40 & -60 & -80 & $\begin{array}{l}-100 \\
\mathrm{ppm}\end{array}$ & -120 & -140 & -160 & -180 & -200 \\
\hline
\end{tabular}




\section{4-(4-methylpiperidin-1-yl)butan-1-ol (S2). $\mathrm{CDCl}_{3}, 400 \mathrm{MHz}$ :}
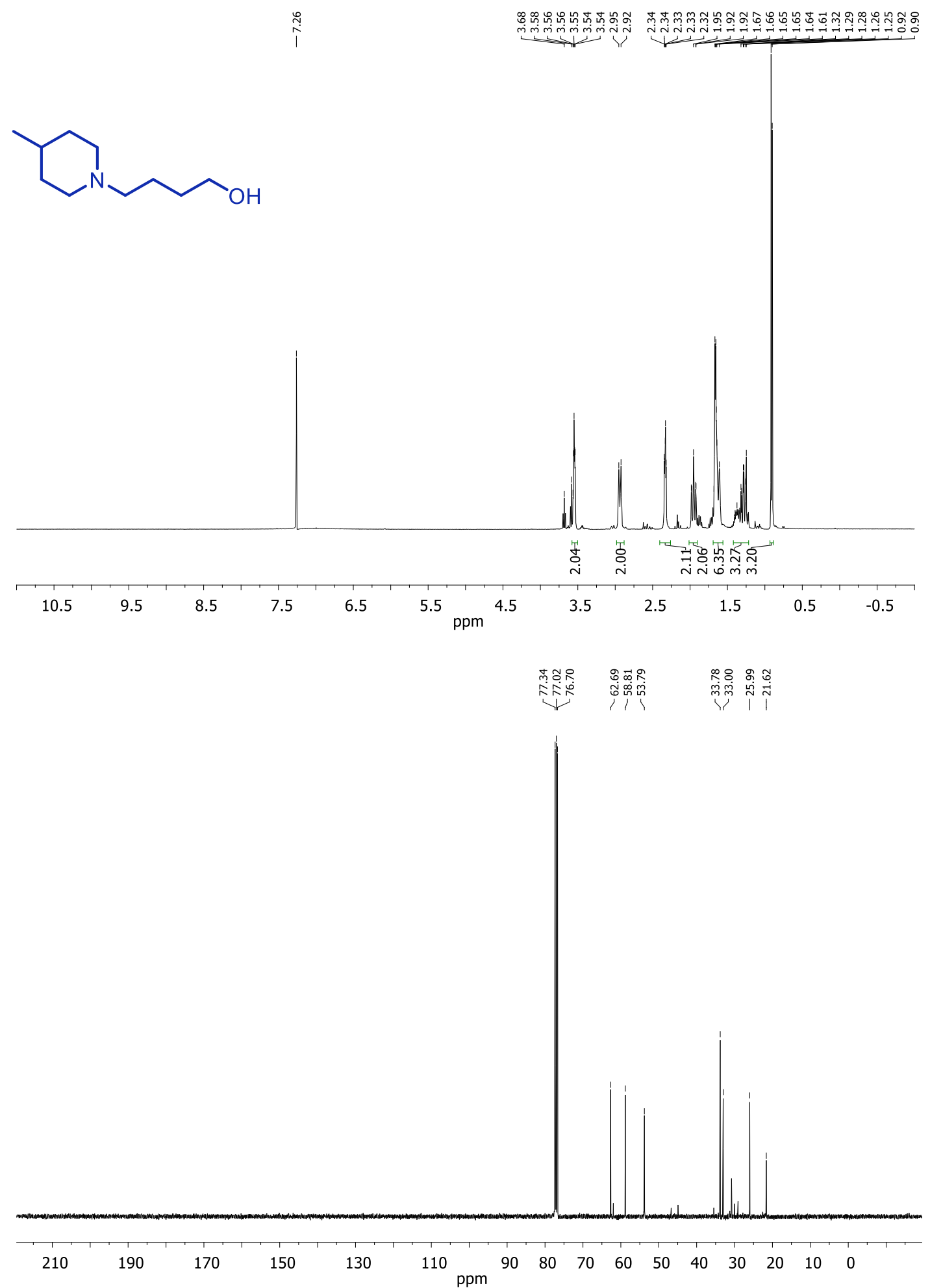
4-(4-chlorophenyl)-1-(4-hydroxybutyl)piperidin-4-ol (S3). DMSO-d $\mathrm{d}_{6}, 400 \mathrm{MHz}$ :

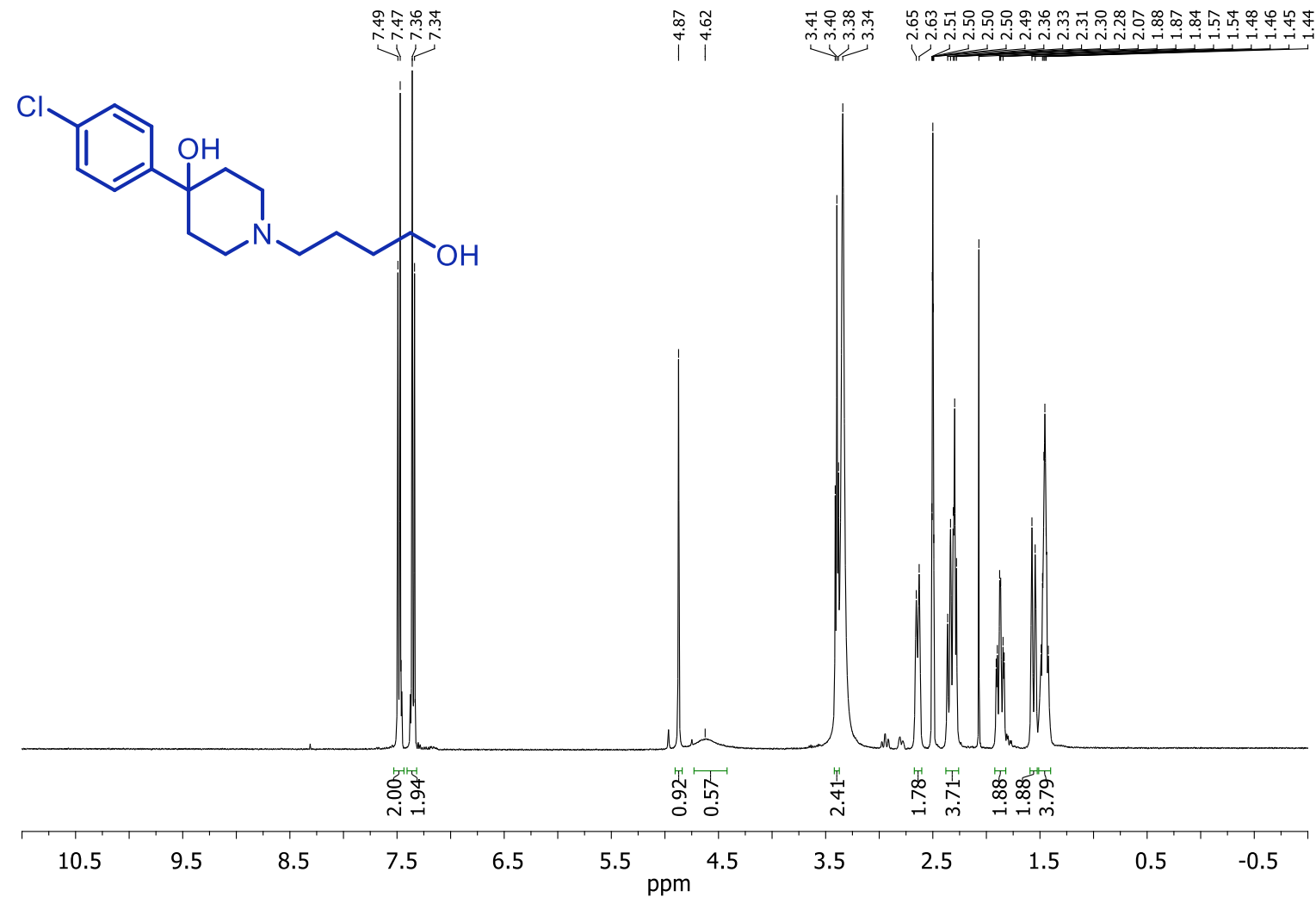

$$
\text { 告 }
$$

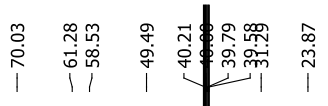

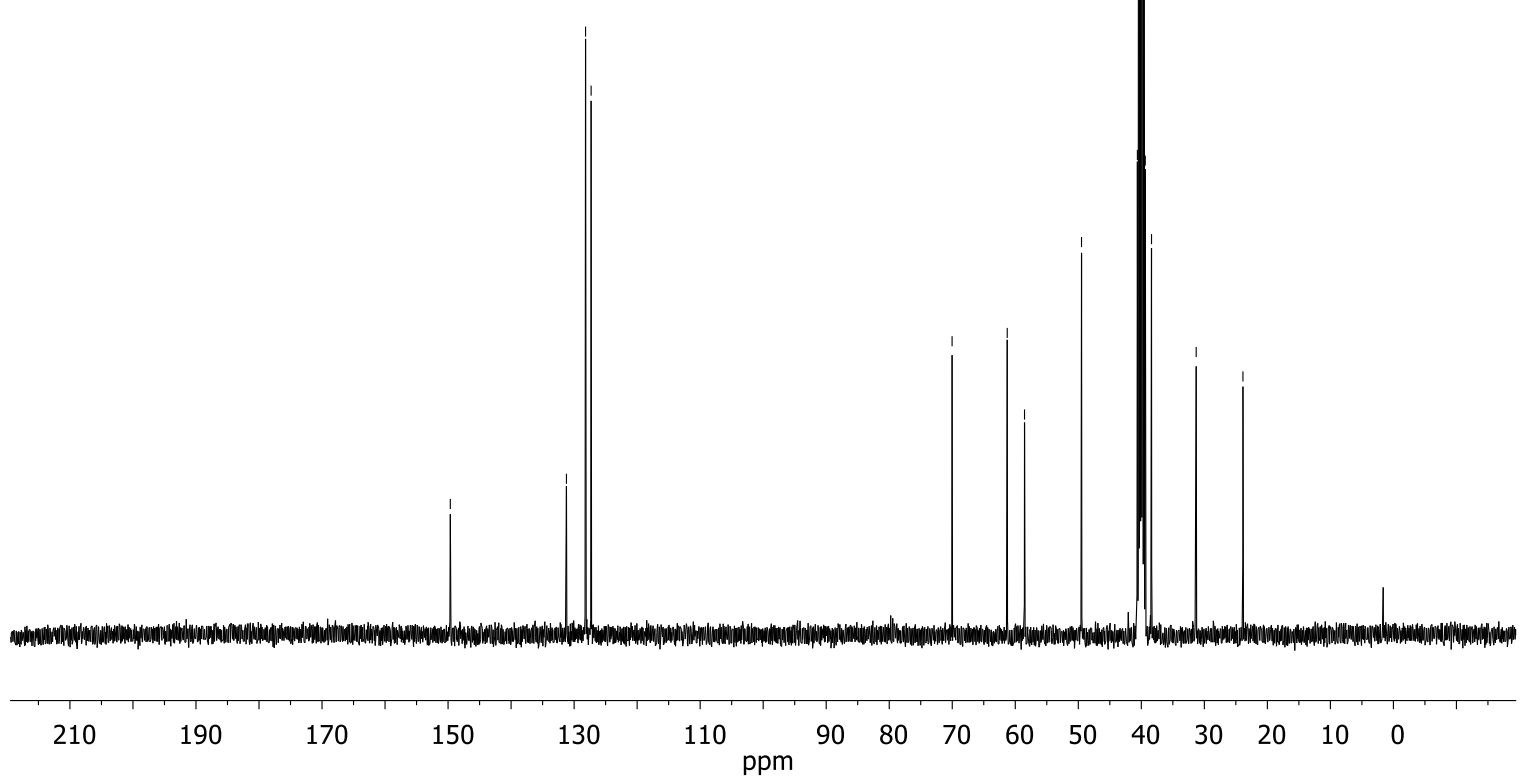


benzophenone (3). $\mathrm{CDCl}_{3}, 400 \mathrm{MHz}$ :

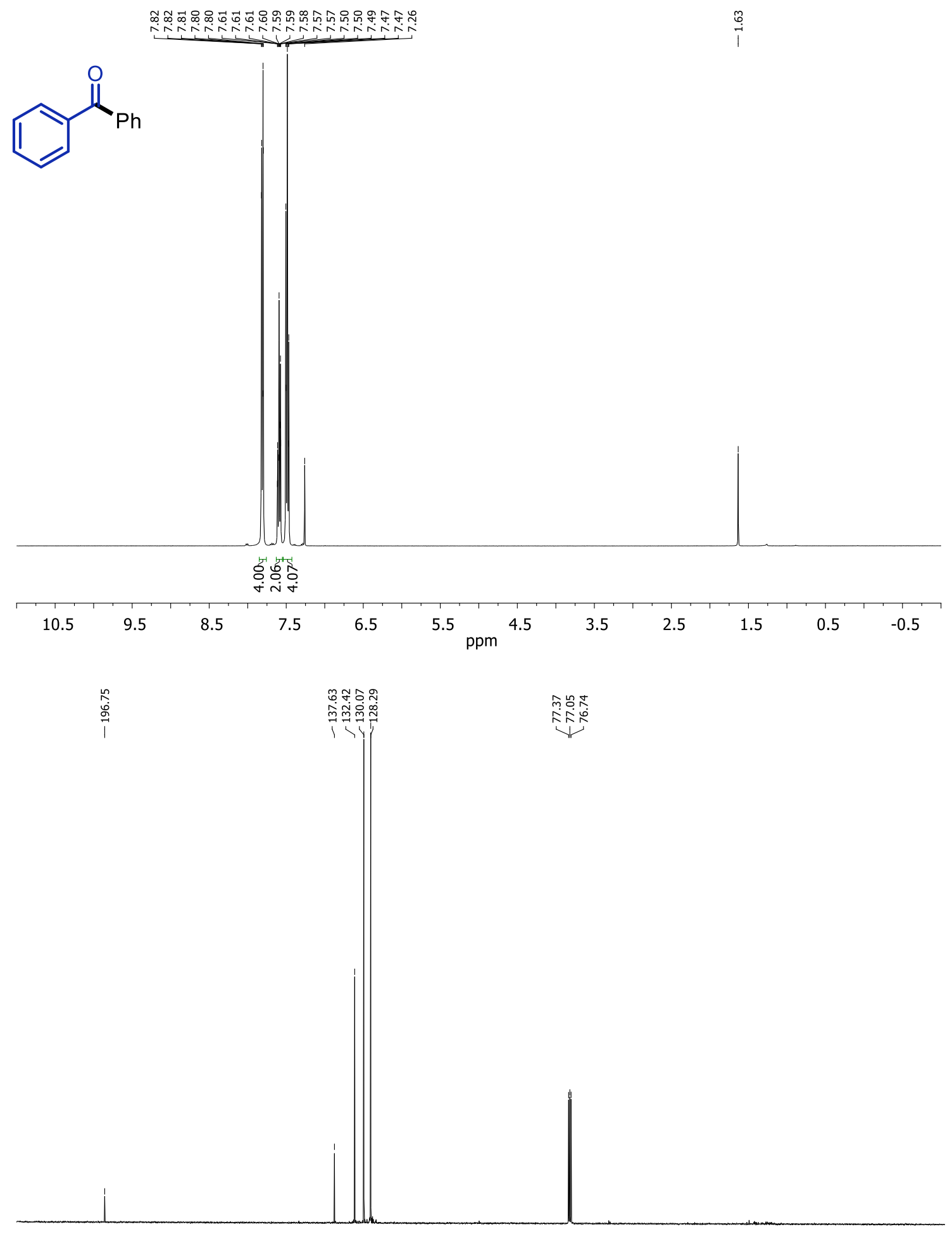

$210 \quad 190 \quad 170 \quad 150 \quad 130 \quad 110 \begin{array}{llllllllllll}\mathrm{ppm} & 90 & 80 & 70 & 60 & 50 & 40 & 30 & 20 & 10 & 0 & \end{array}$


2-benzoylnaphthalene (4). $\mathrm{CDCl}_{3}, 400 \mathrm{MHz}$ :

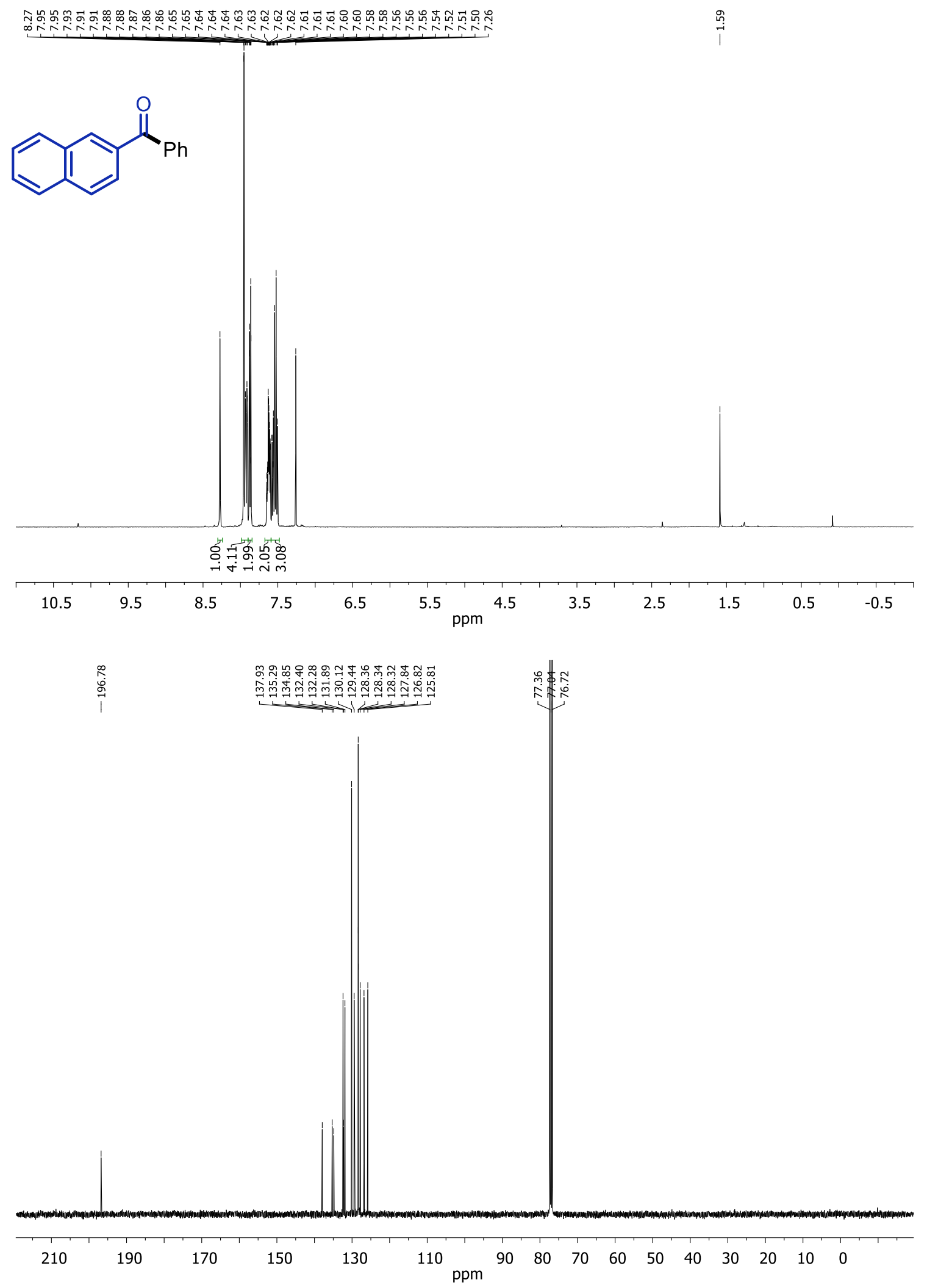


4-methylbenzophenone (5). $\mathrm{CDCl}_{3}, 400 \mathrm{MHz}$ :

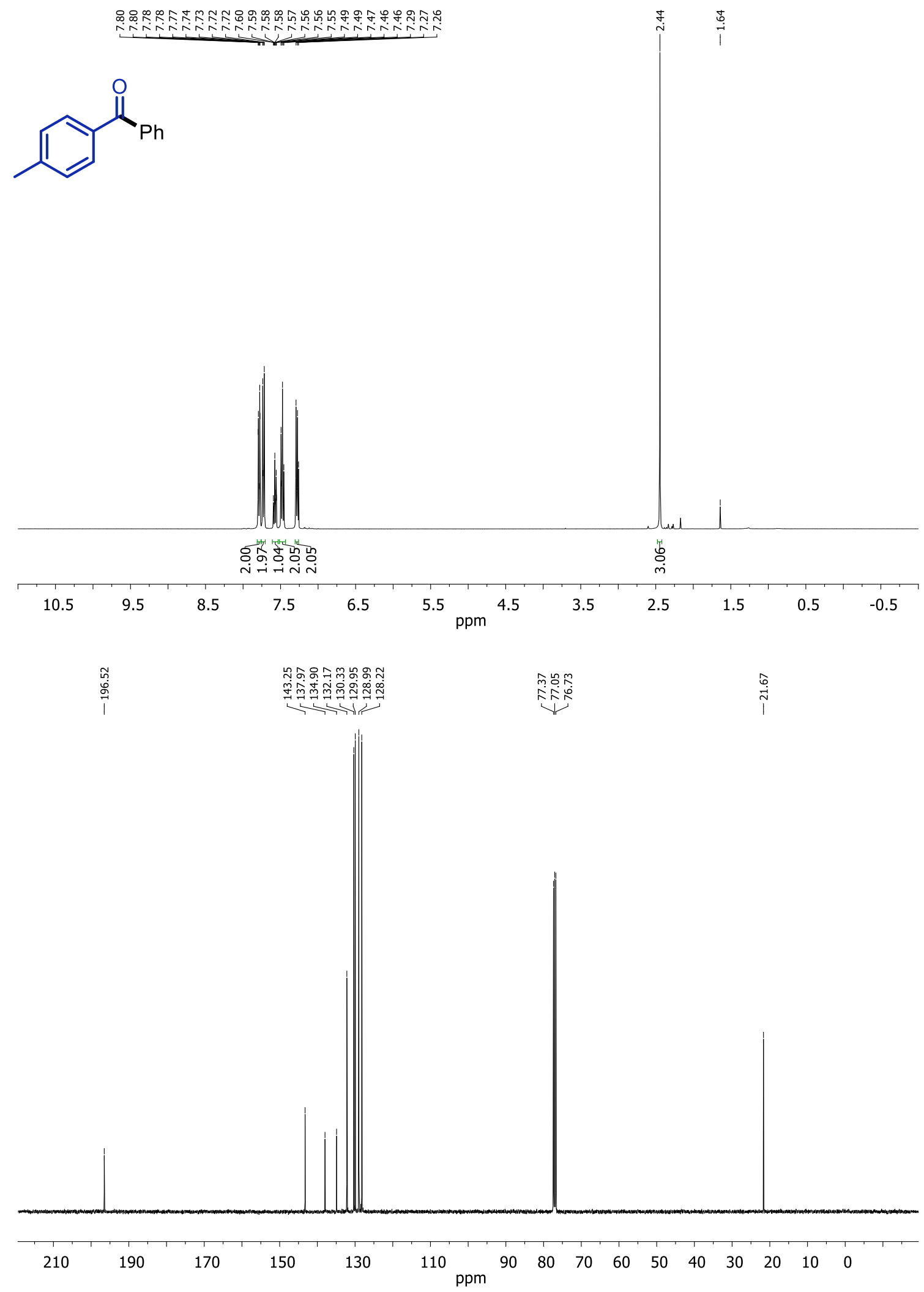


4-methoxybenzophenone (6). $\mathrm{CDCl}_{3}, 400 \mathrm{MHz}$ :

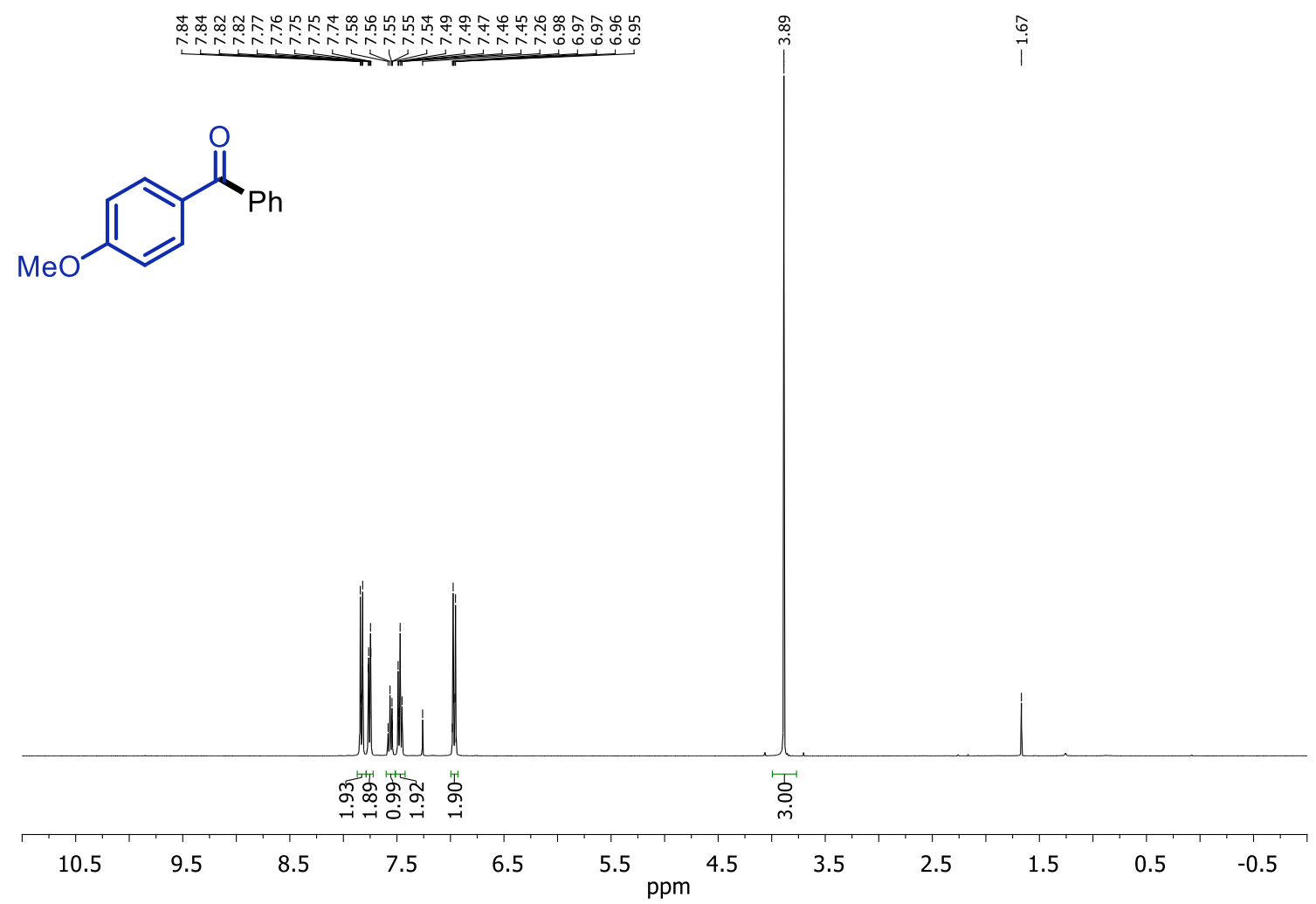

|

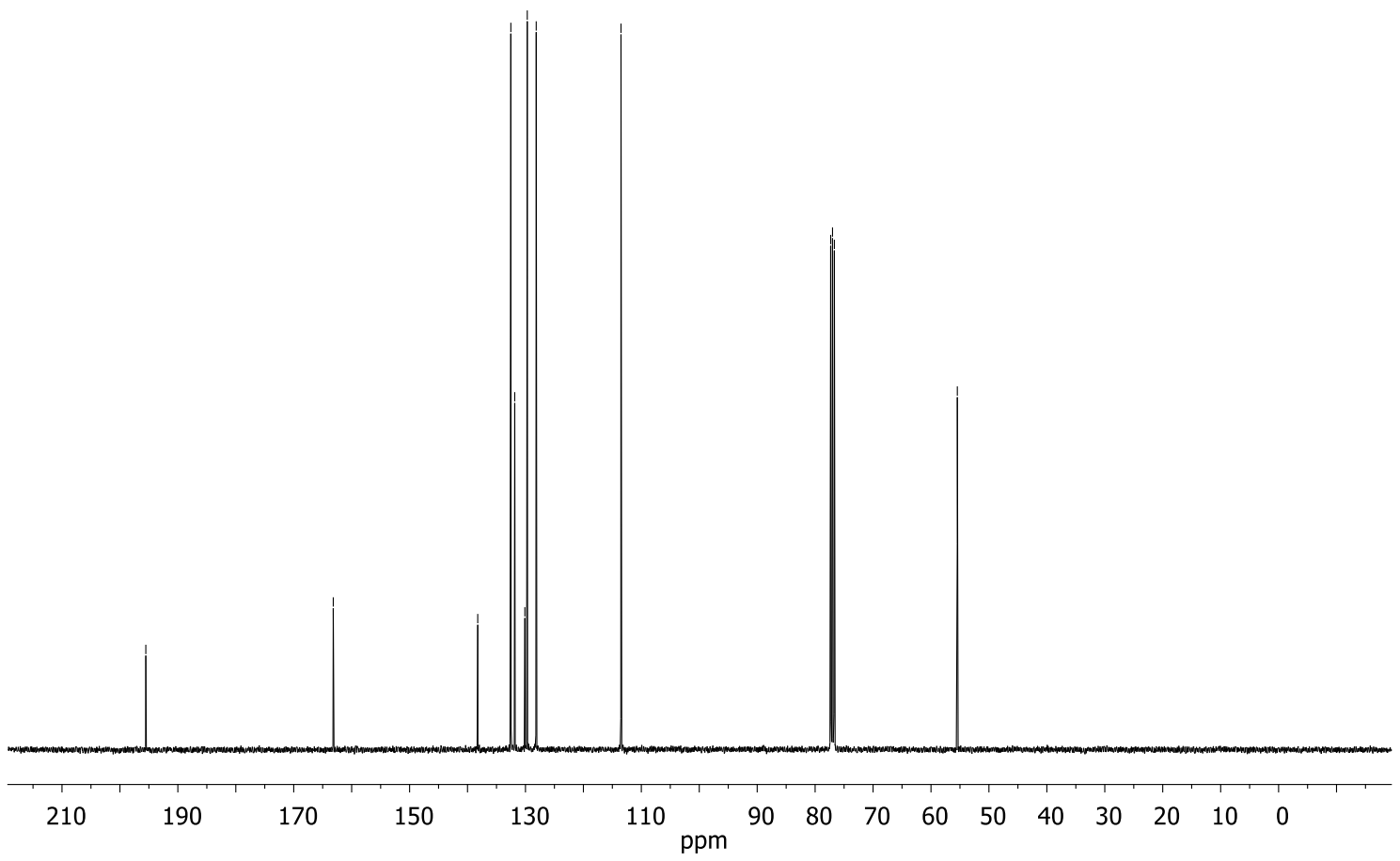


3-methoxybenzophenone (7). $\mathrm{CDCl}_{3}, 400 \mathrm{MHz}$ :
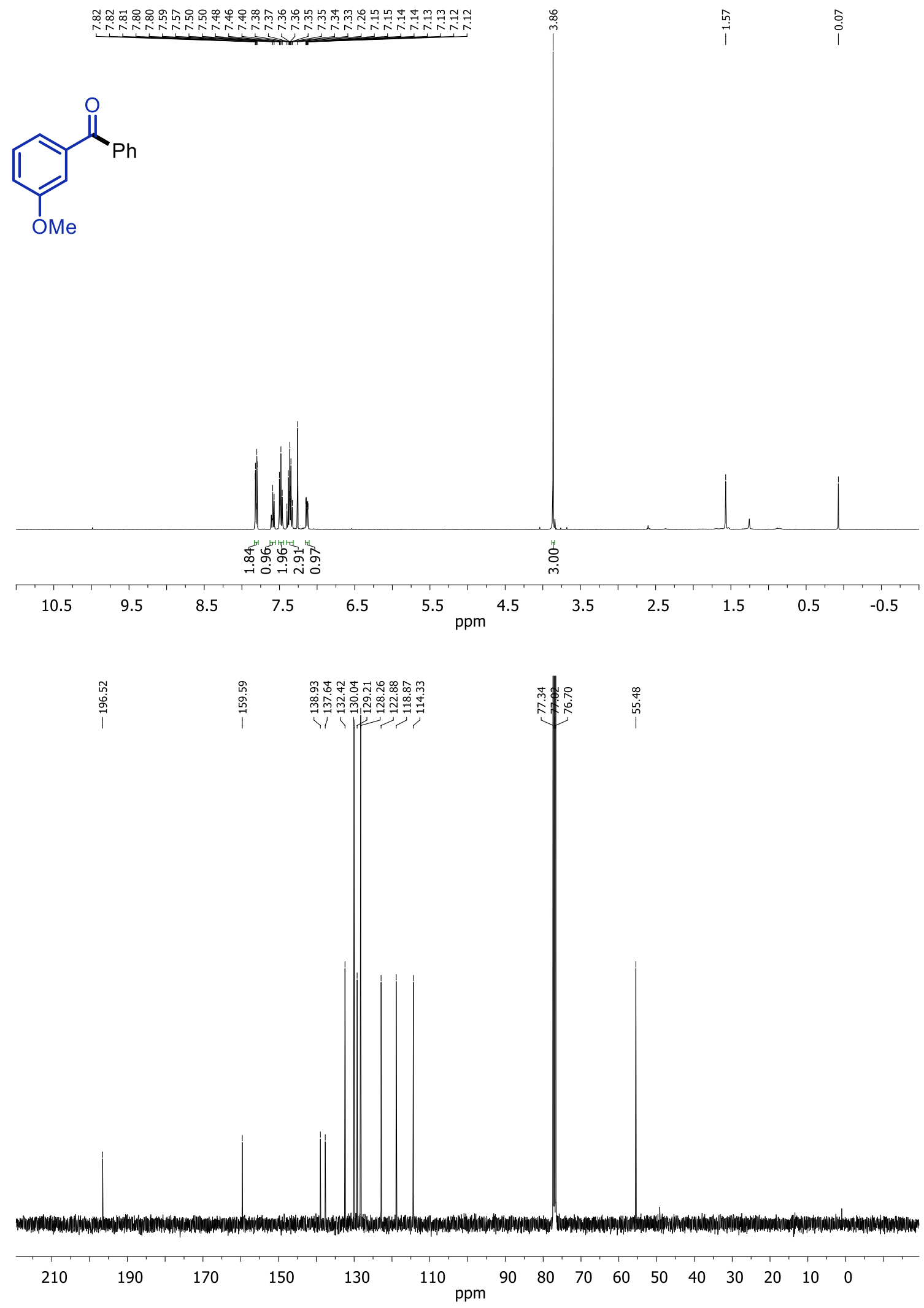
benzo[d][1,3]dioxol-4-yl(phenyl)methanone (8). $\mathrm{CDCl}_{3}, 400 \mathrm{MHz}$ :

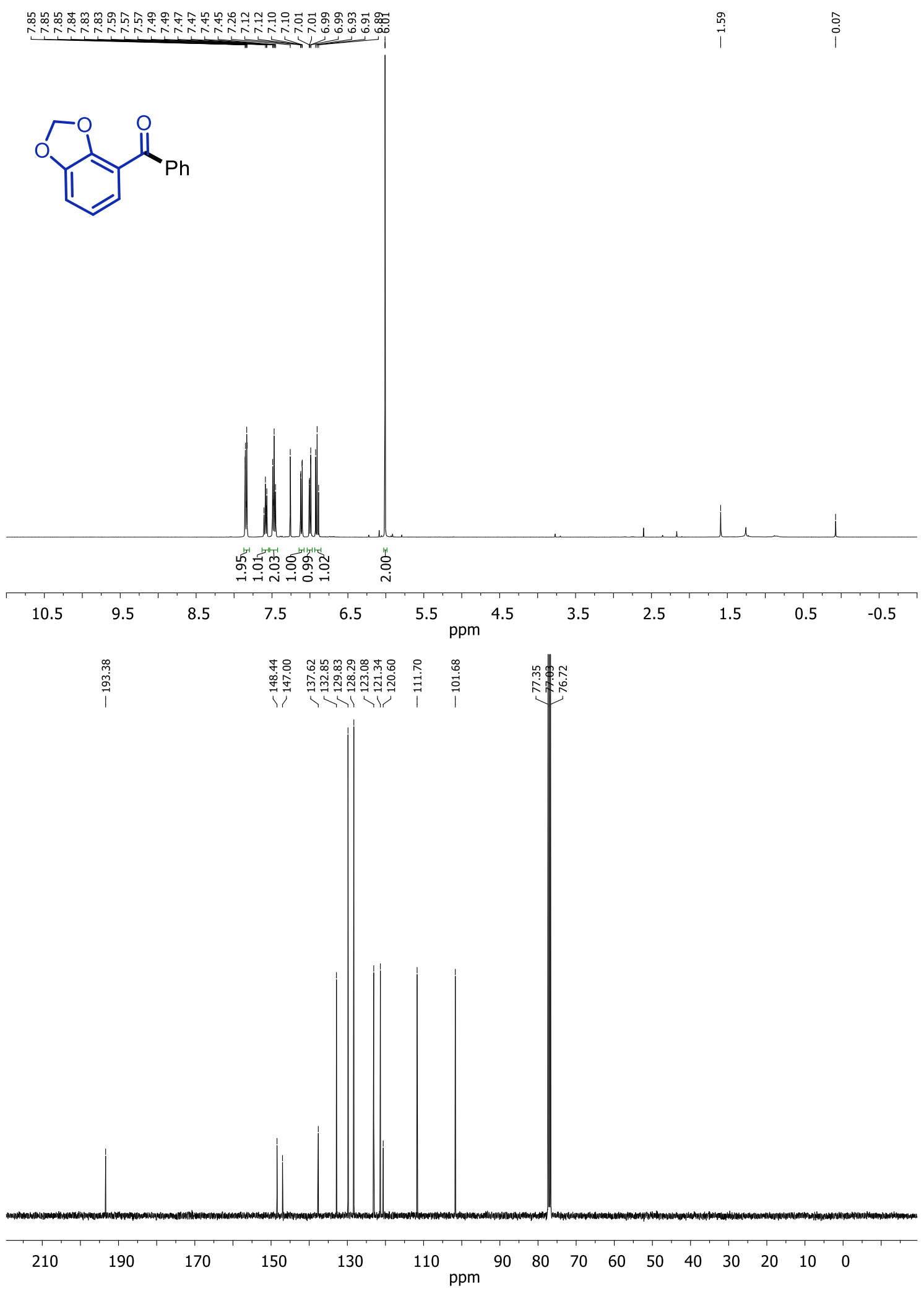


4-fluorobenzophenone (9). $\mathrm{CDCl}_{3}, 400 \mathrm{MHz}$ :
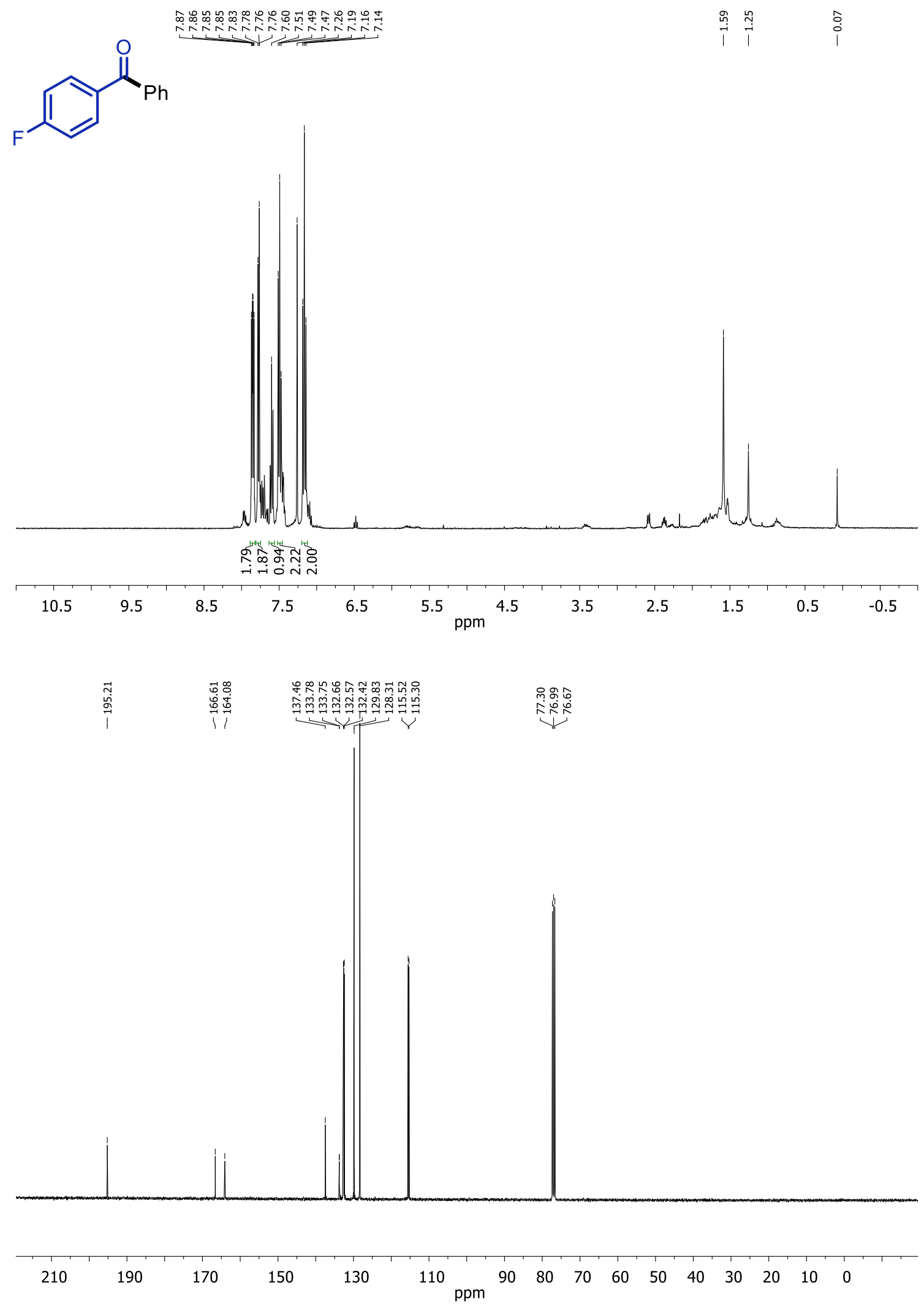
4-(trifluoromethyl)benzophenone (10). $\mathrm{CDCl}_{3}, 400 \mathrm{MHz}$ :
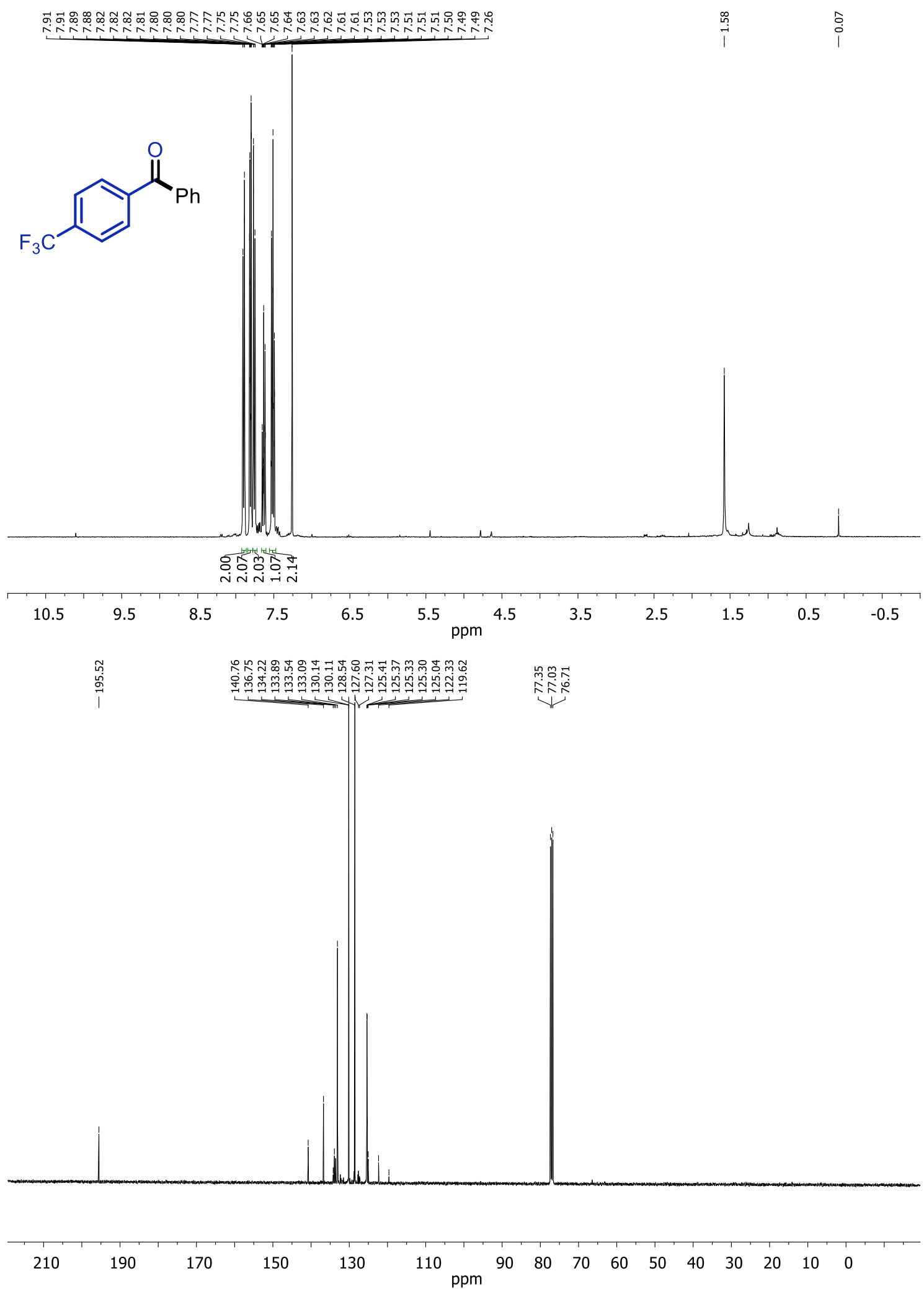
1,4-dibenzoylbenzene (11). $\mathrm{CDCl}_{3}, 400 \mathrm{MHz}$ :
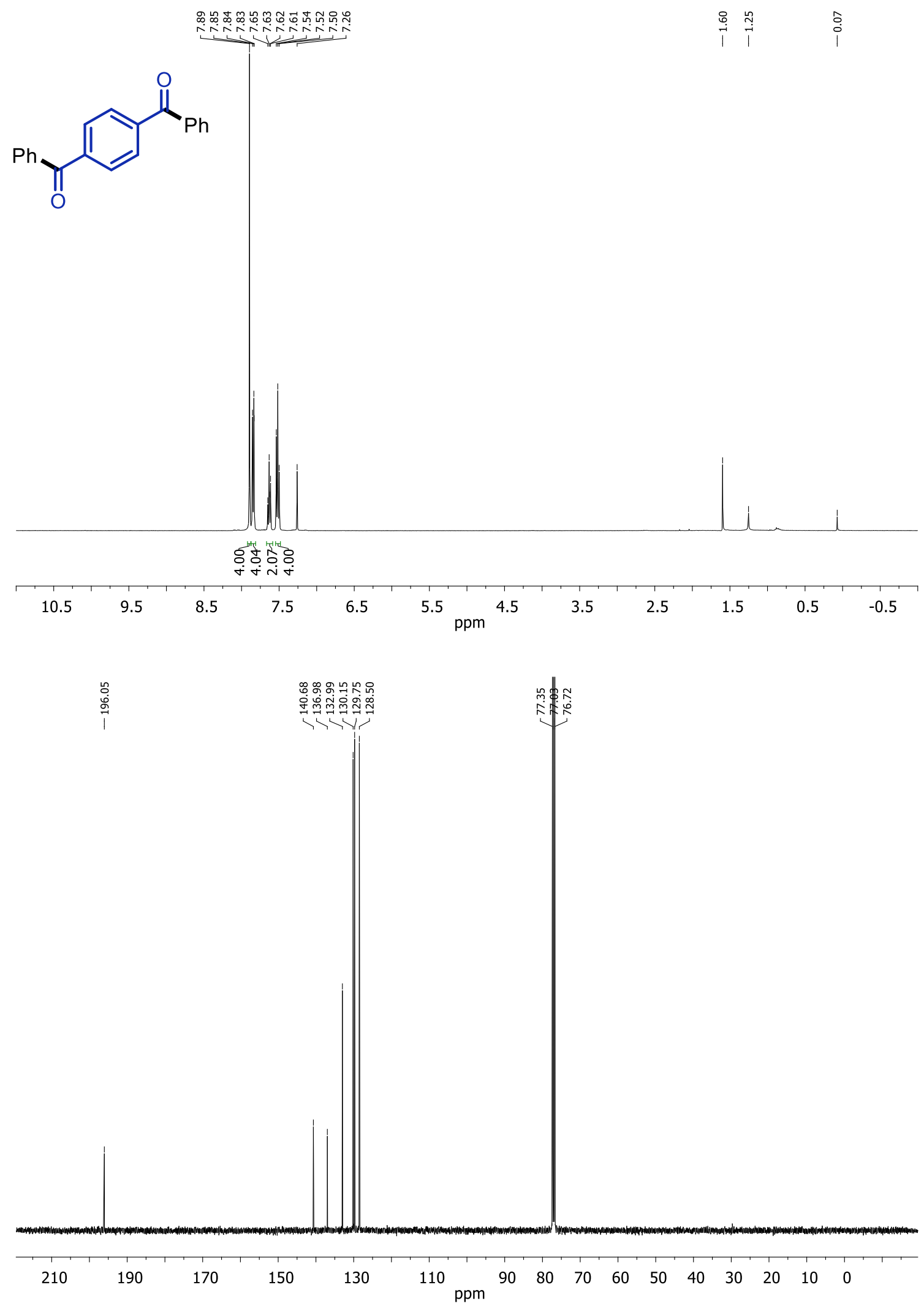
[1,1'-biphenyl]4-yl(phenyl)methanone (12). $\mathrm{CDCl}_{3}, 400 \mathrm{MHz}$ :
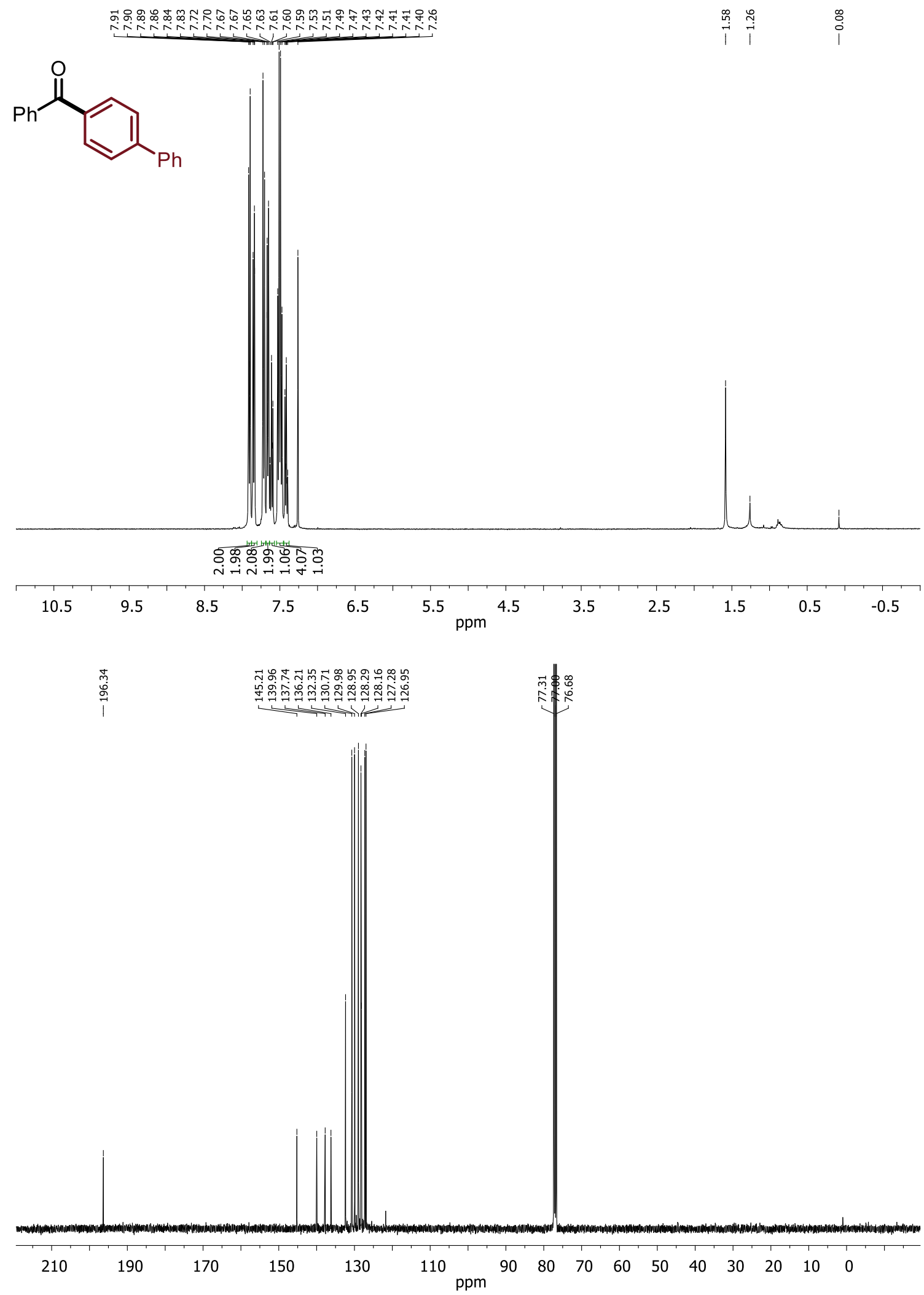
2-benzoylnaphthalene (13). $\mathrm{CDCl}_{3}, 400 \mathrm{MHz}$ :
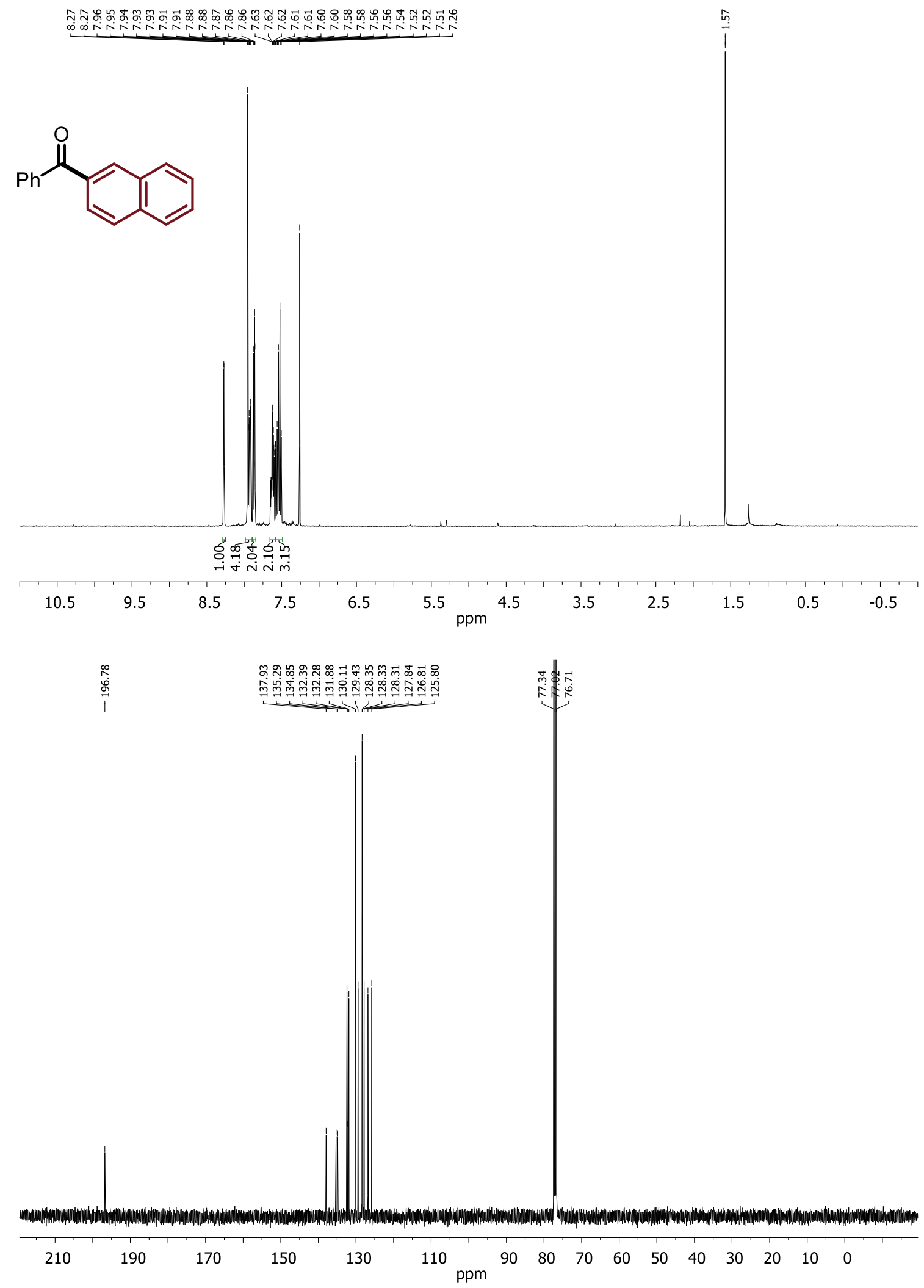
4-methoxynaphthalen-1-yl) (phenyl)methanone (14). $\mathrm{CDCl}_{3}, 400 \mathrm{MHz}$ :
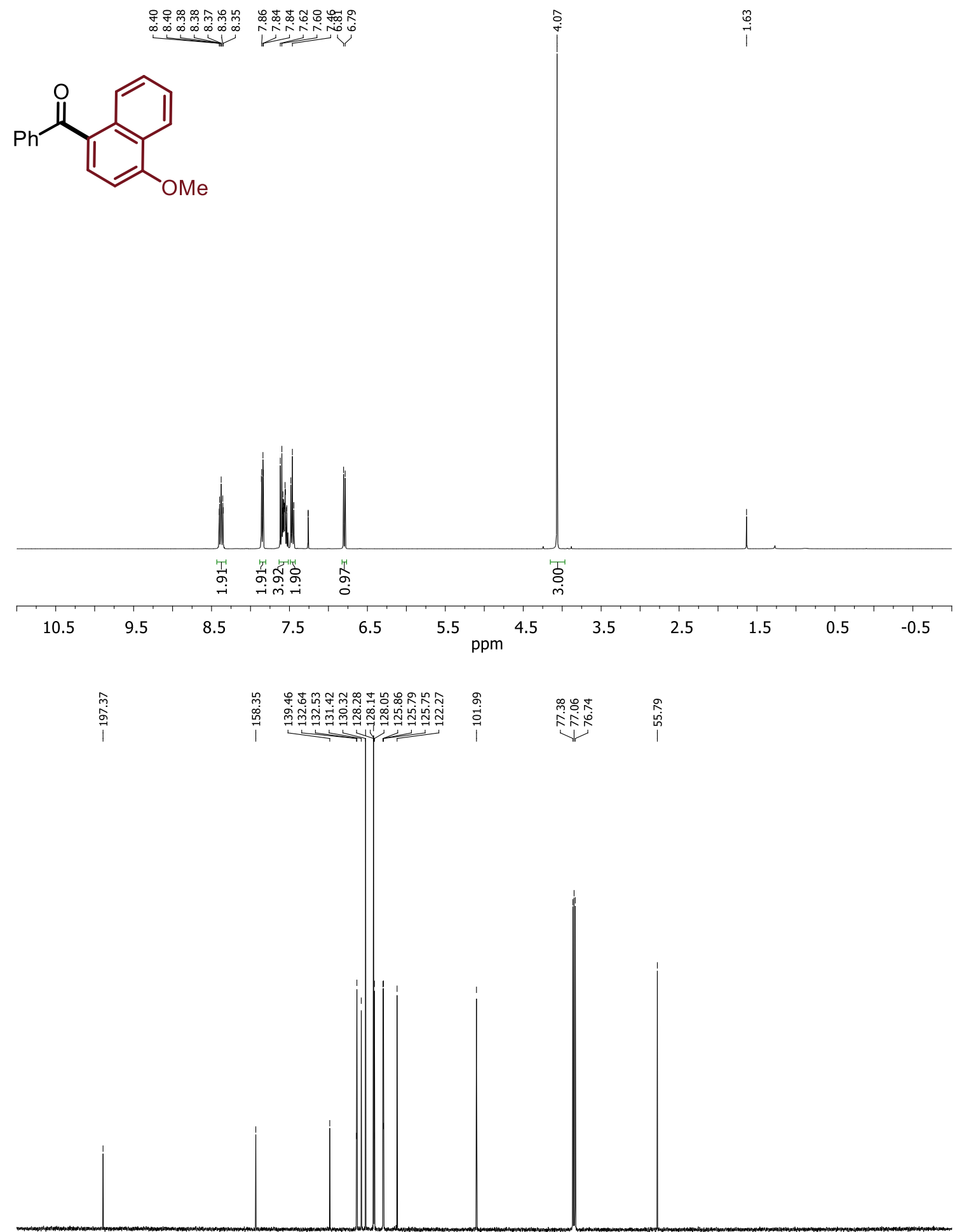

210

190

170

150

130

110

$\begin{array}{llllllllll}90 & 80 & 70 & 60 & 50 & 40 & 30 & 20 & 10 & 0\end{array}$ 
4-acetylbenzophenone (17). $\mathrm{CDCl}_{3}, 400 \mathrm{MHz}$ :
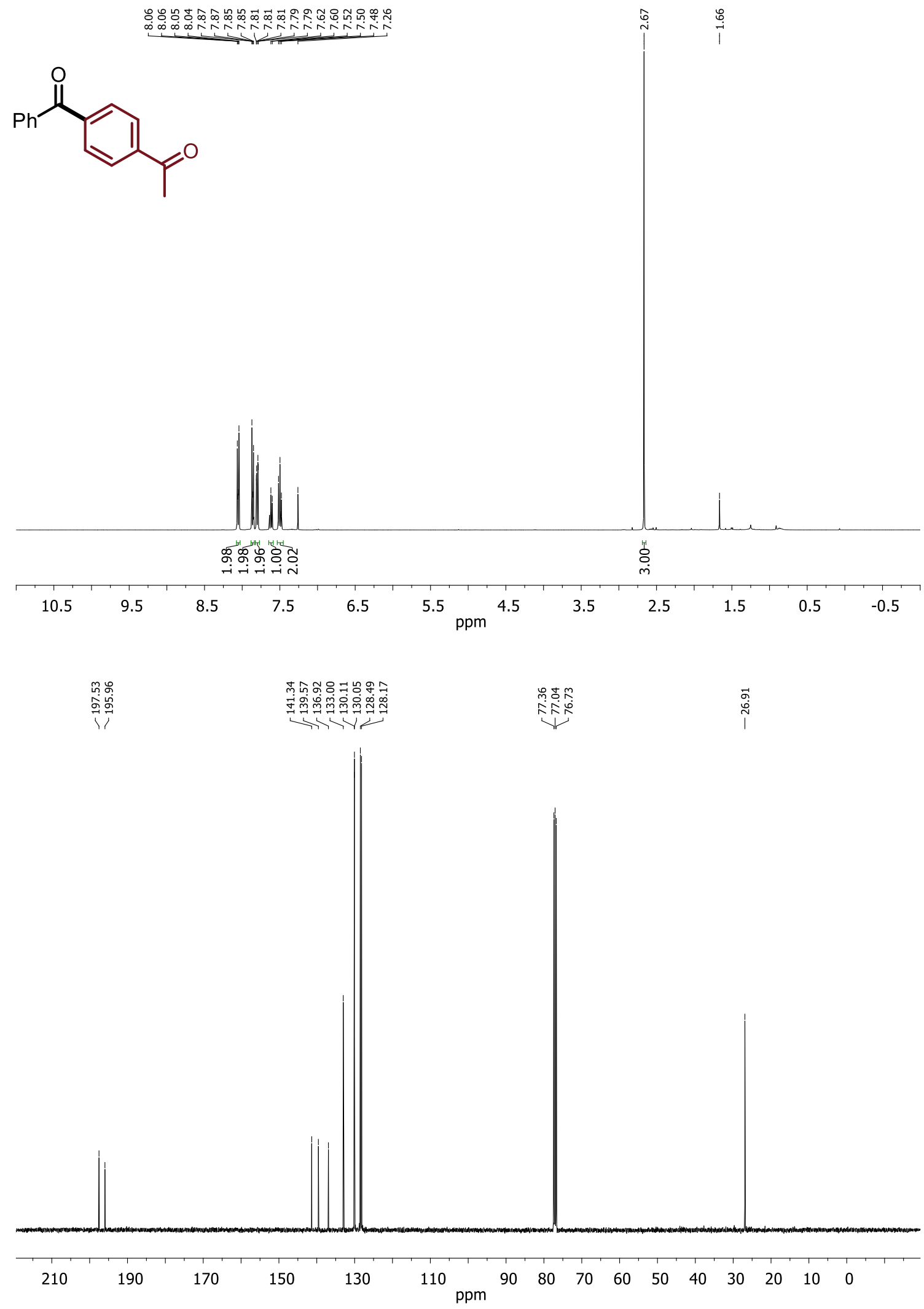
(2,6-dimethylphenyl)(phenyl)methanone (18). $\mathrm{CDCl}_{3}, 400 \mathrm{MHz}$ :
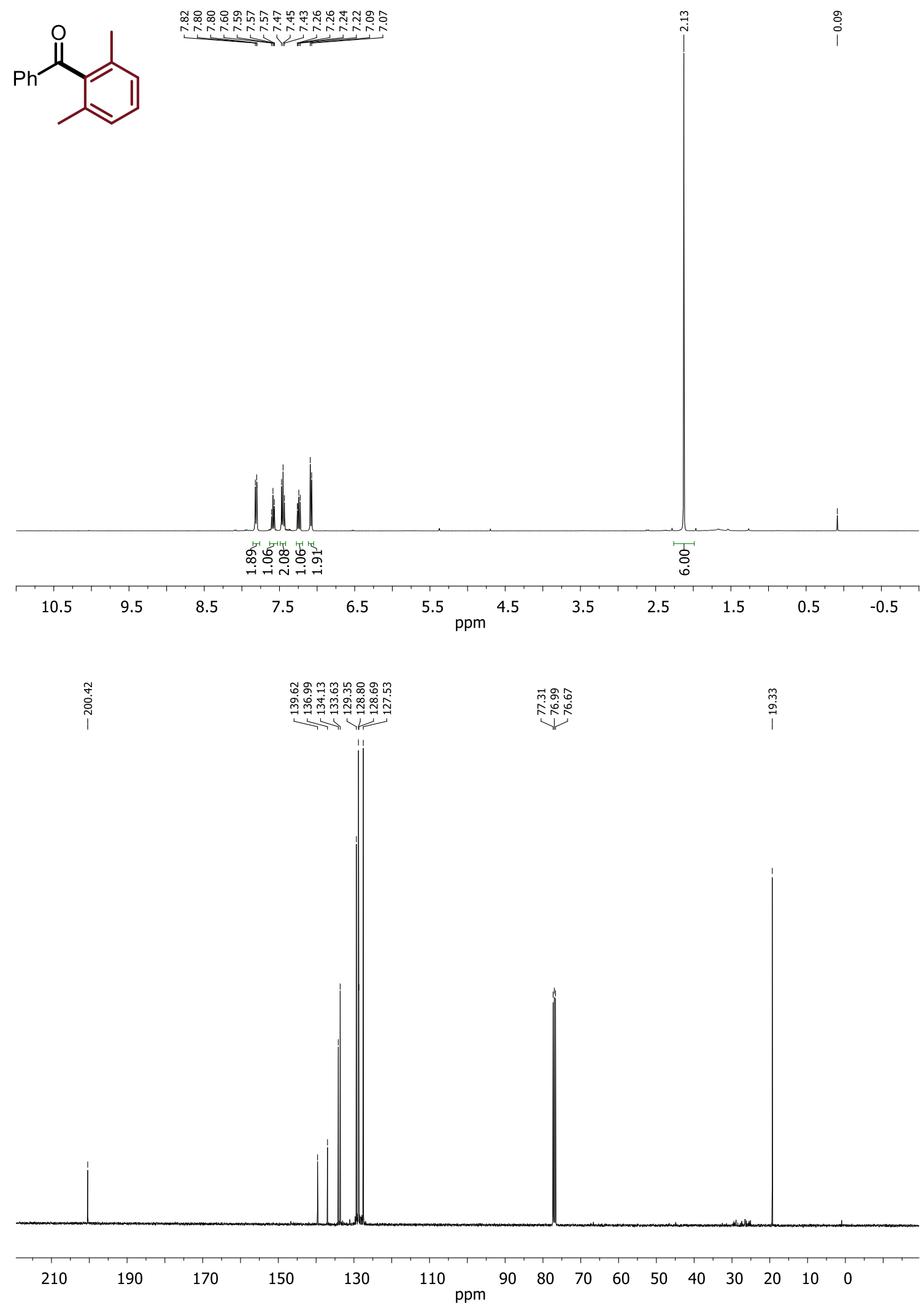
4-chlorobenzophenone (19). $\mathrm{CDCl}_{3}, 400 \mathrm{MHz}$ :

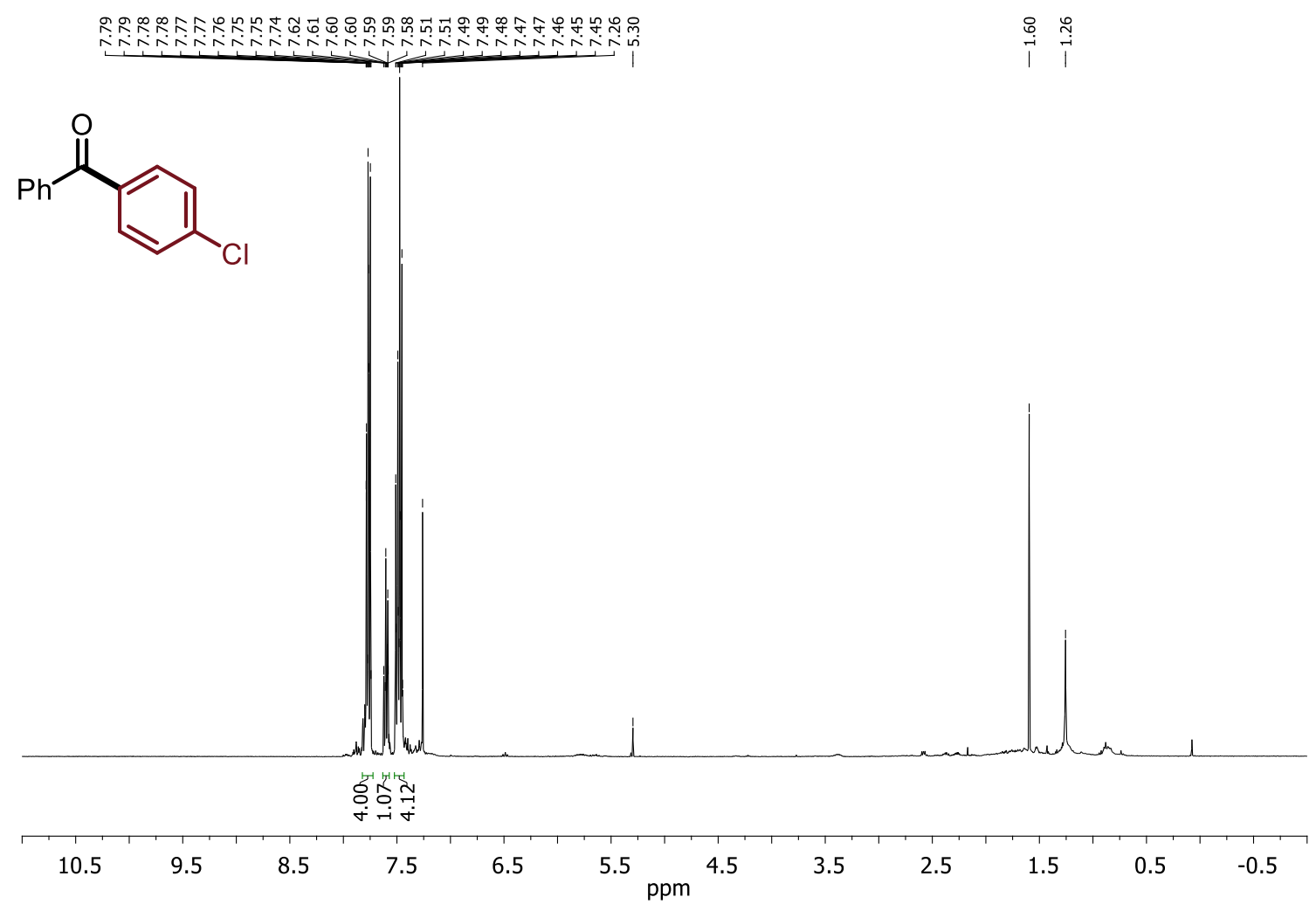

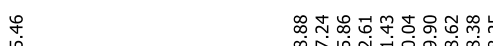

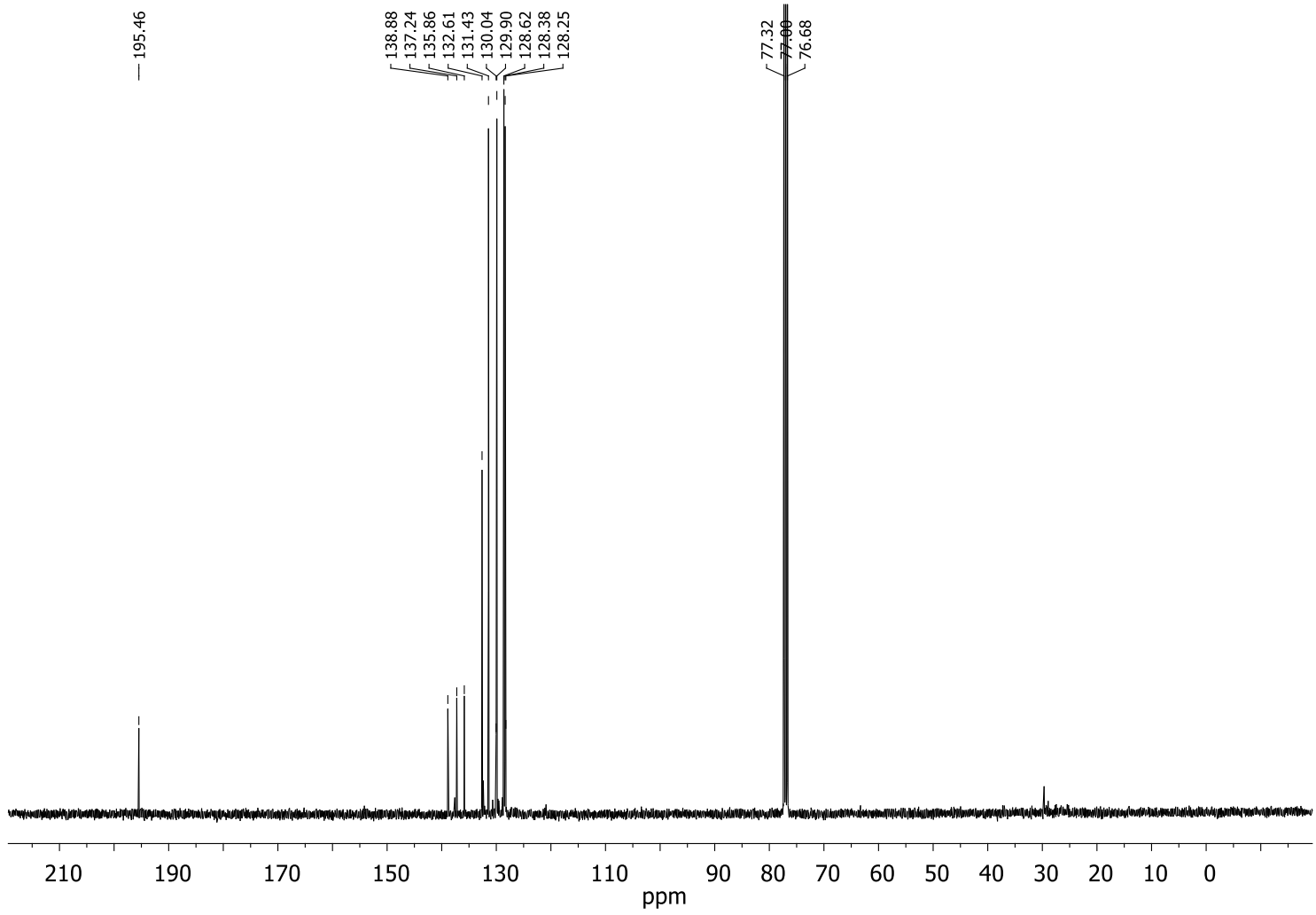


N-(4-benzoylphenyl)acetamide (20). $\mathrm{CDCl}_{3}, 400 \mathrm{MHz}$ :
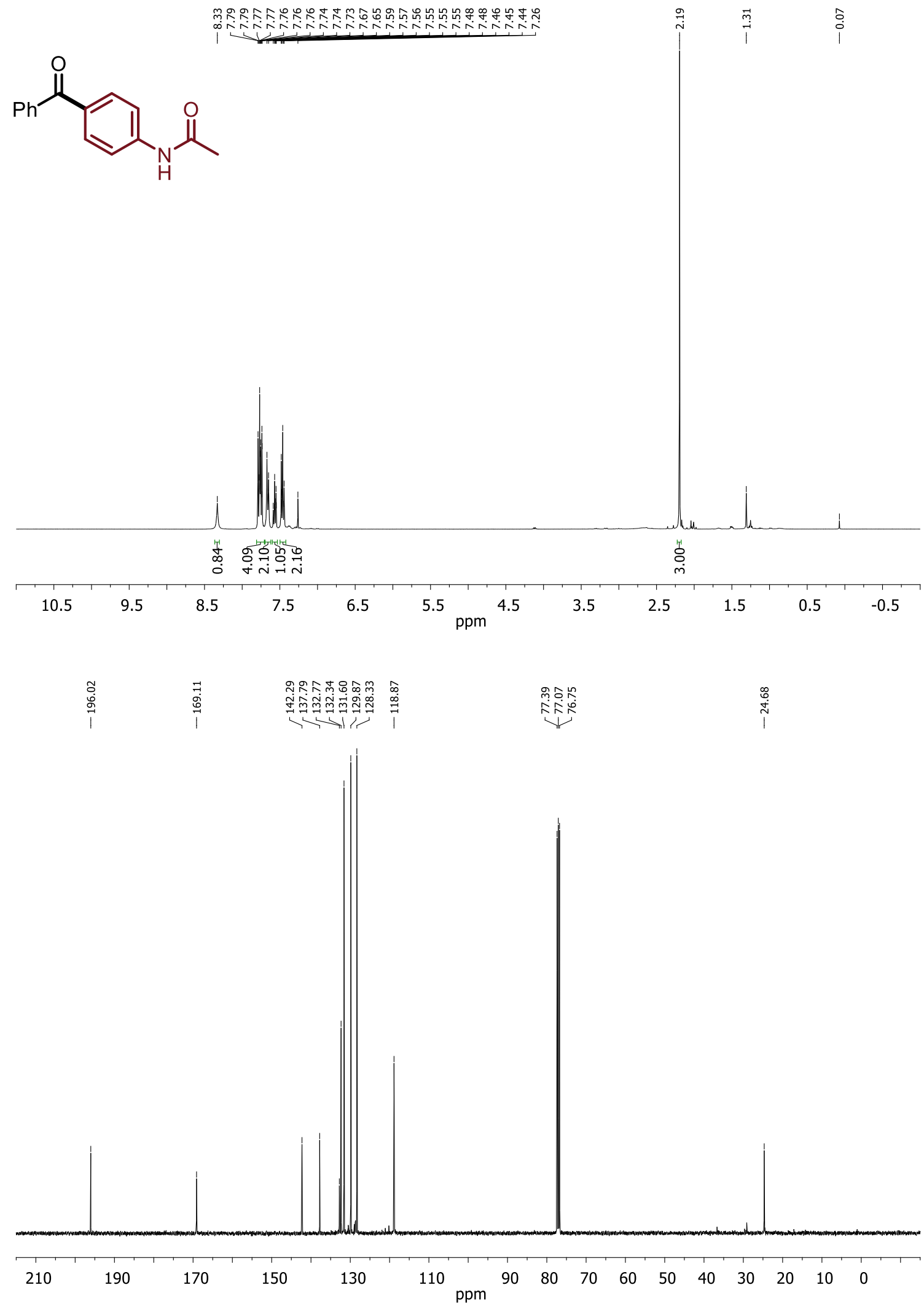
3-benzoylestra-1,3,5(10)-trien-17-one (21). $\mathrm{CDCl}_{3}, 400 \mathrm{MHz}$ :

ळ

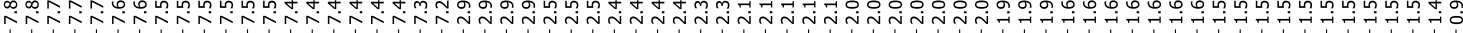<smiles>C[C@]12CC[C@@H]3c4ccc(C(=O)c5ccccc5)cc4CC[C@H]3[C@@H]1CCC2=O</smiles>
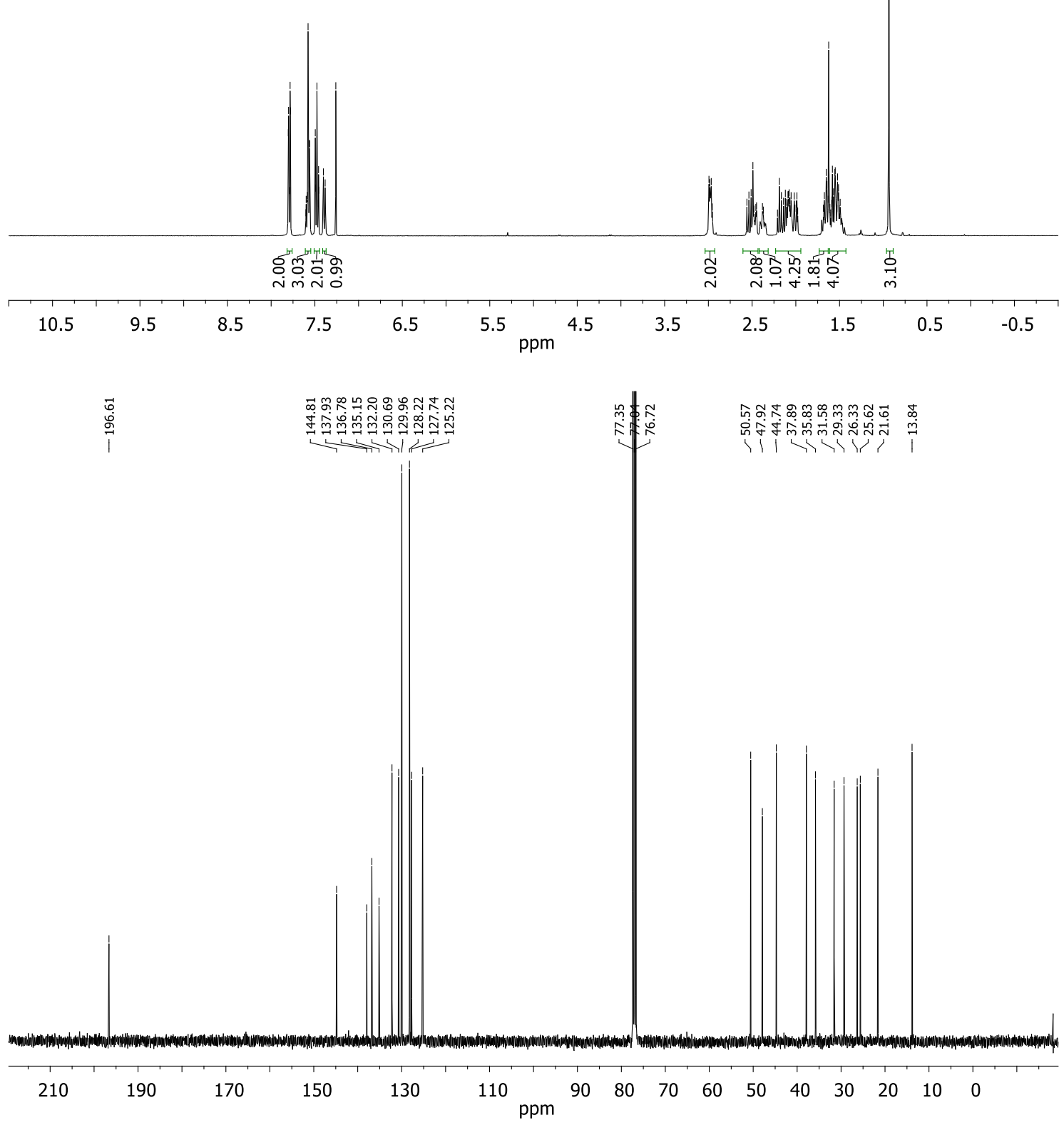
1-phenyl-3-(pyridin-3-yl)propan-1-one (22). $\mathrm{CDCl}_{3}, 400 \mathrm{MHz}$ :
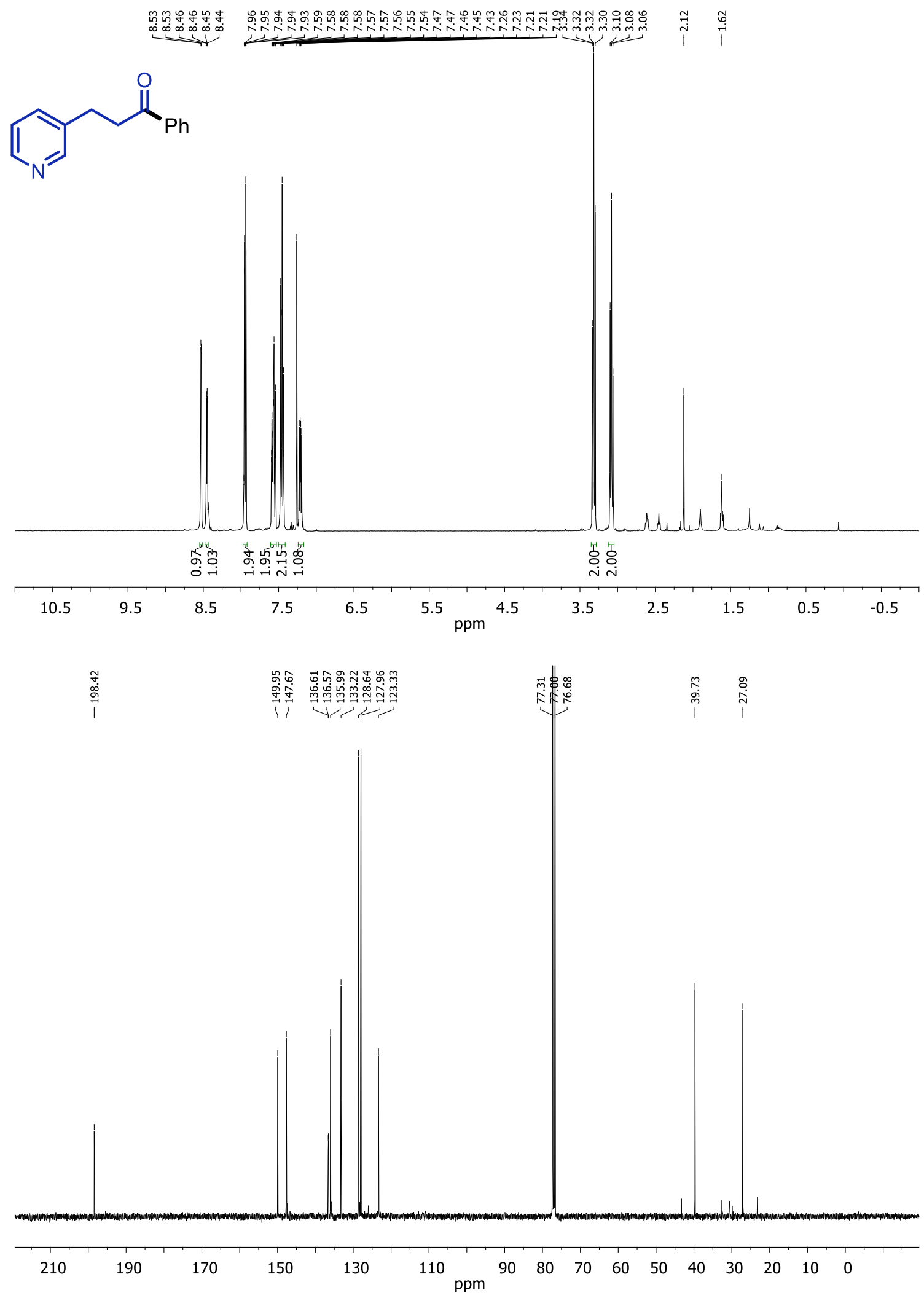
2-(1H-indol-3-yl)-1-phenylethanone (23). $\mathrm{CDCl}_{3}, 400 \mathrm{MHz}$ :
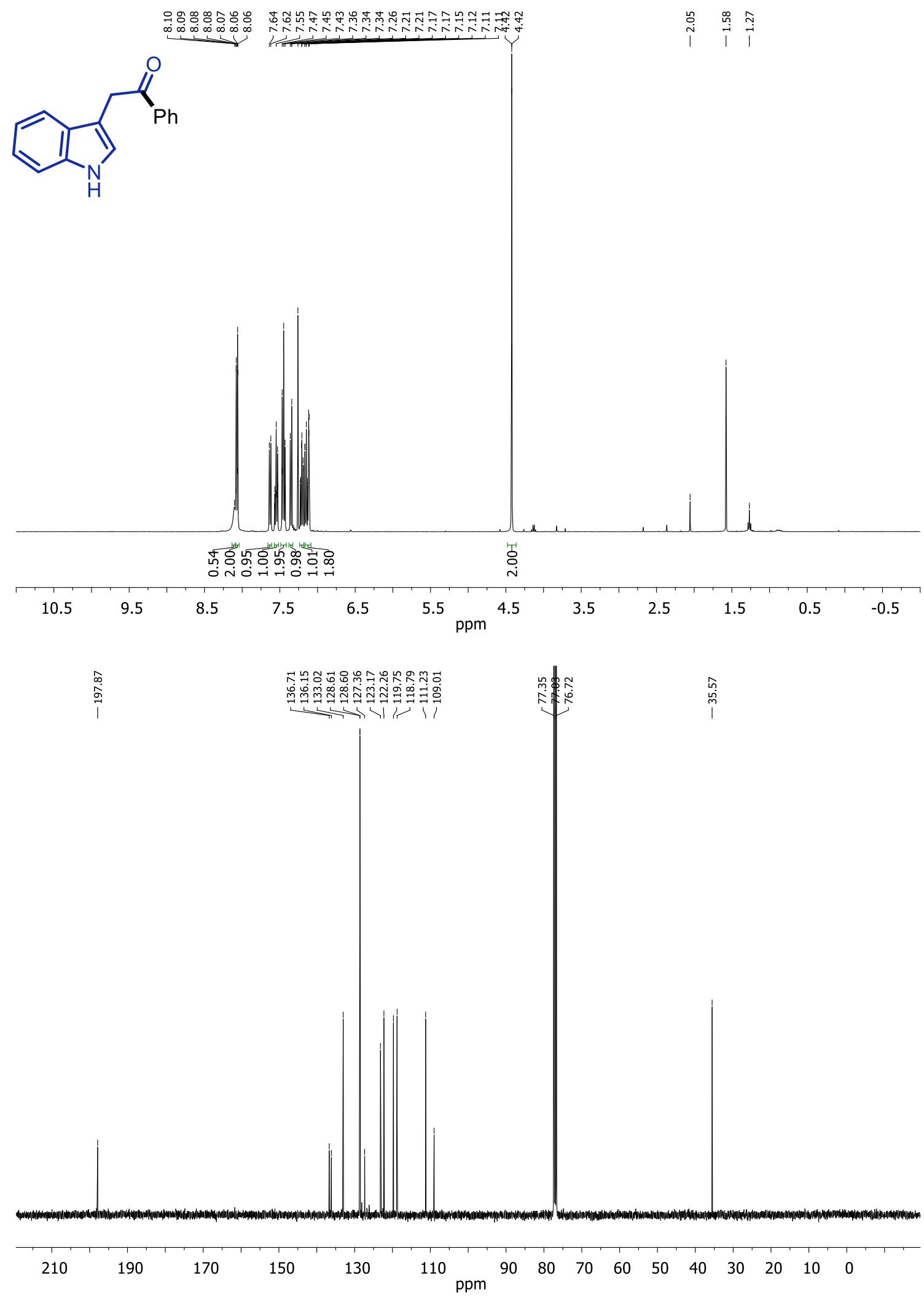
2-furyl(phenyl)methanone (24). $\mathrm{CDCl}_{3}, 400 \mathrm{MHz}$ :
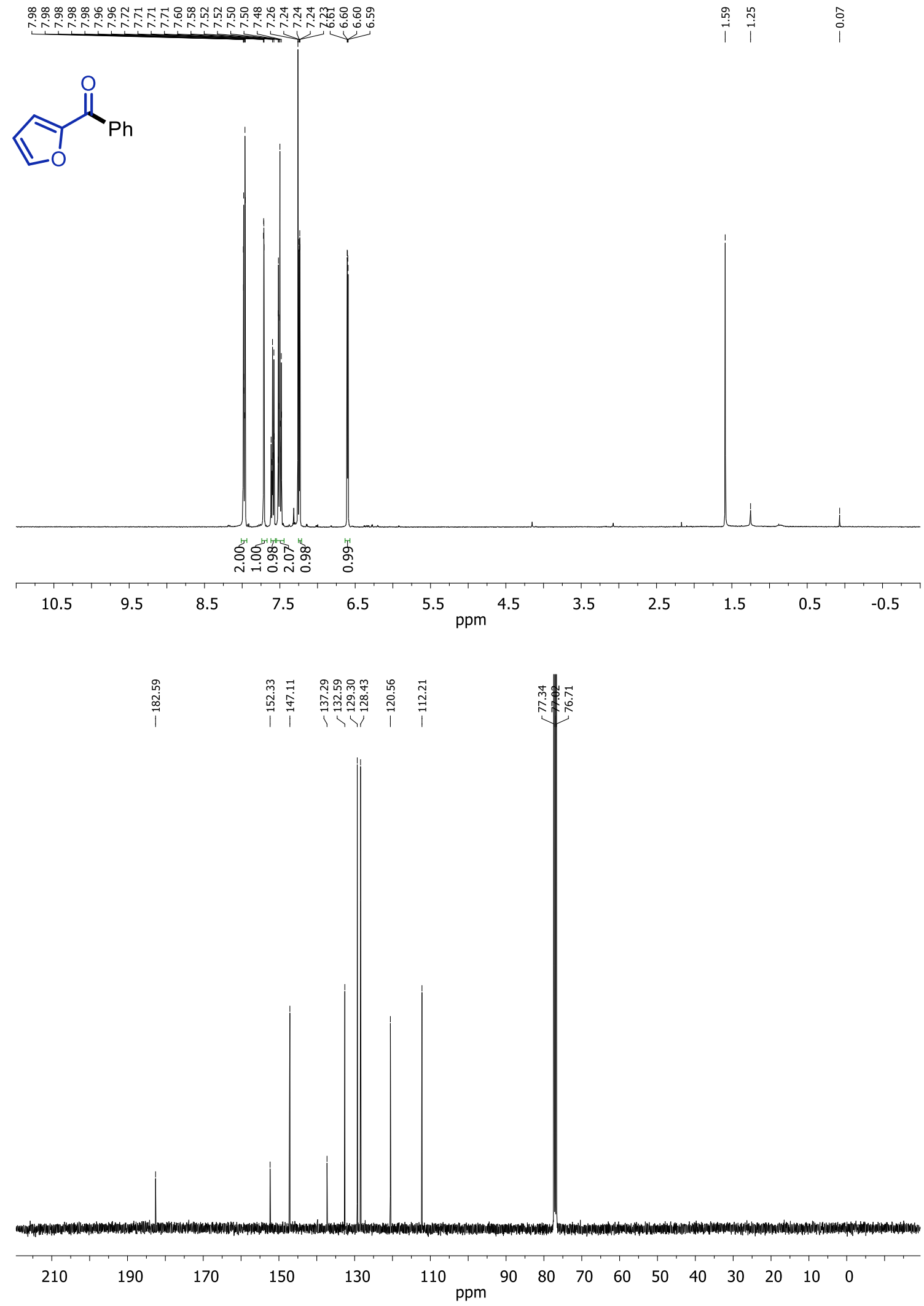
2-benzoylthiophene (25). $\mathrm{CDCl}_{3}, 400 \mathrm{MHz}$ :
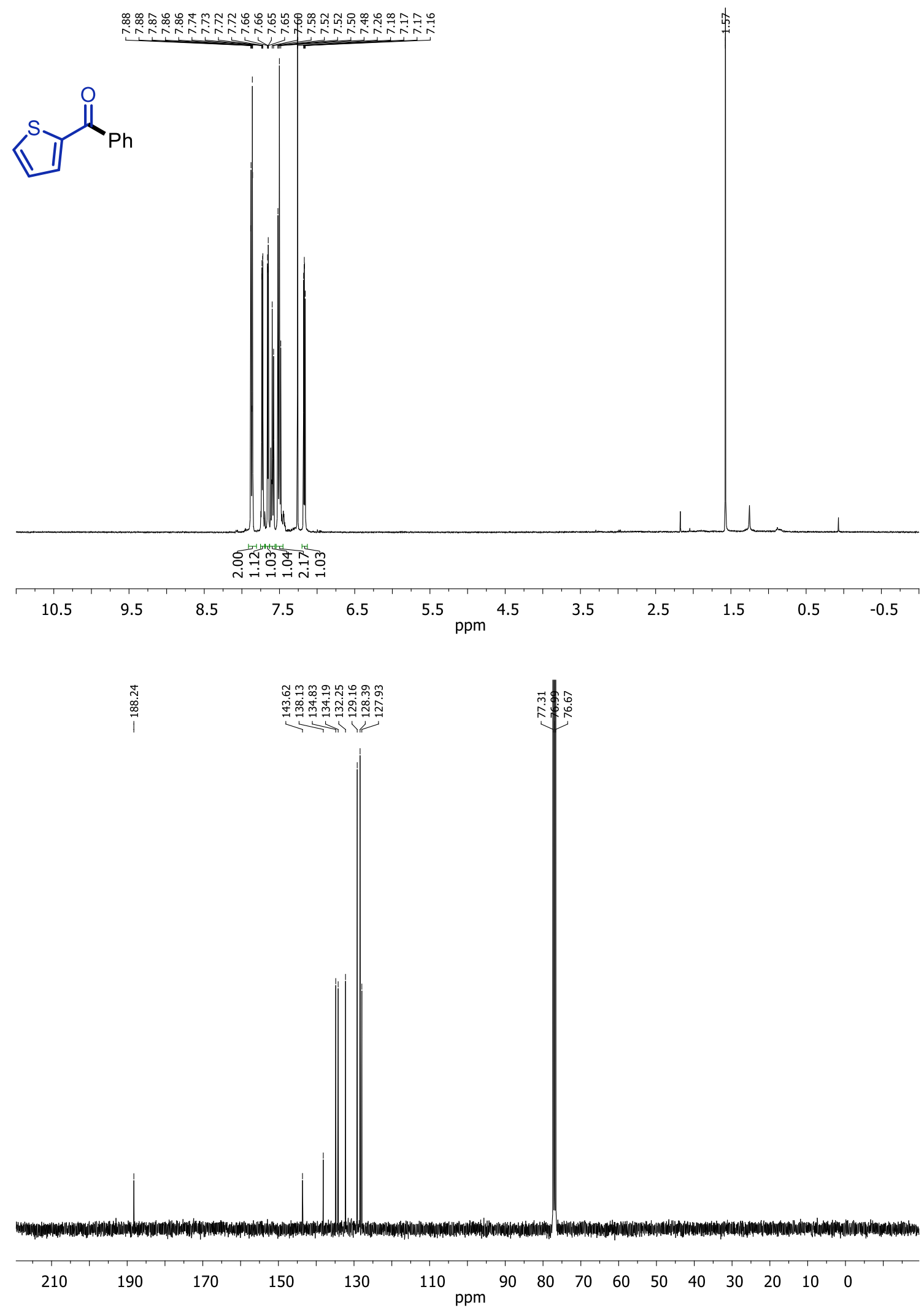
phenyl(quinolin-6-yl)methanone (26). $\mathrm{CDCl}_{3}, 400 \mathrm{MHz}$ :<smiles>O=C(c1ccccc1)c1ccc2ncccc2c1</smiles>

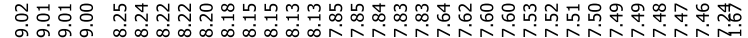

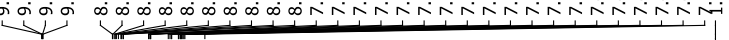
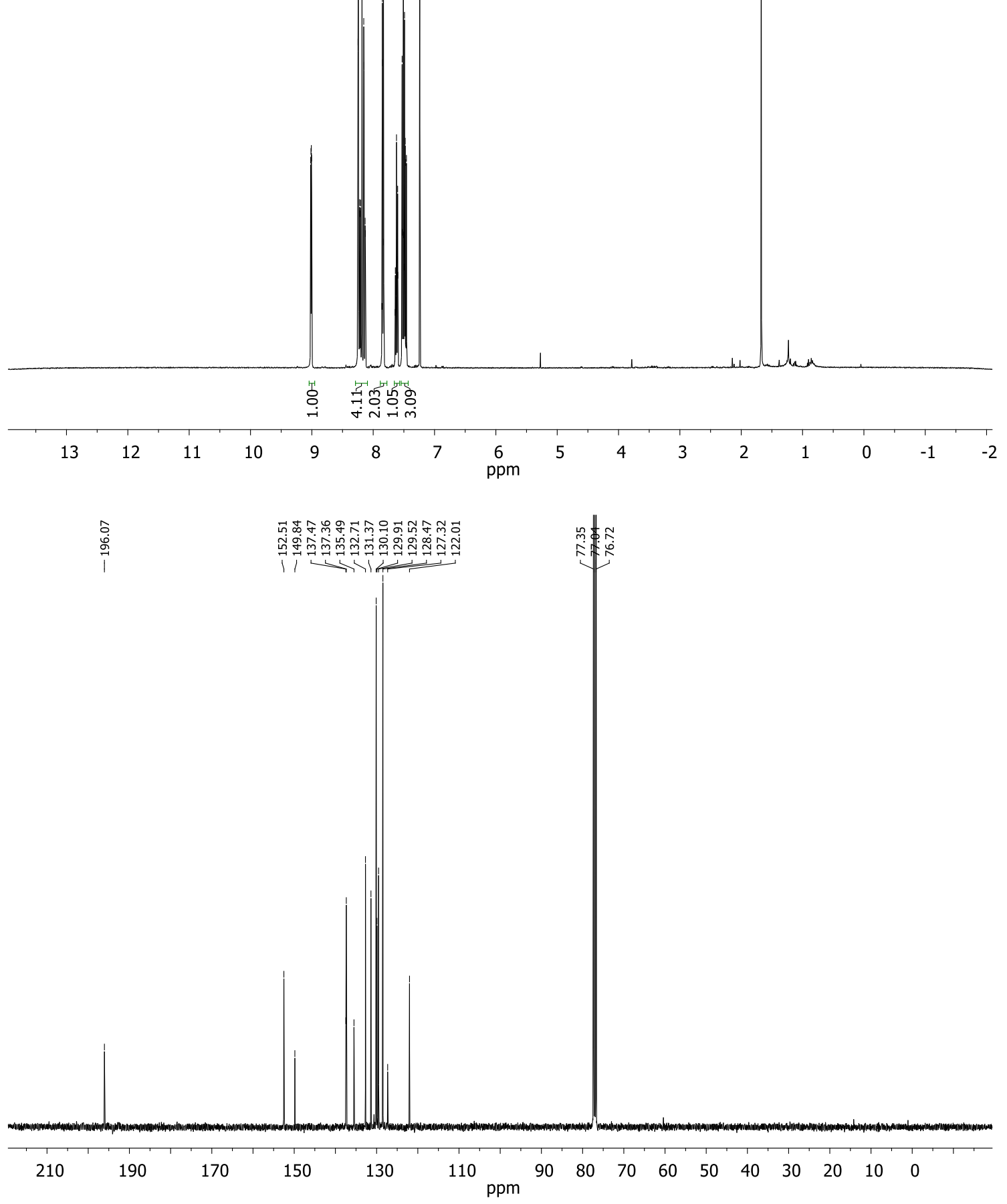
phenyl(pyridine-3-yl)methanone (27). $\mathrm{CDCl}_{3}, 400 \mathrm{MHz}$ :
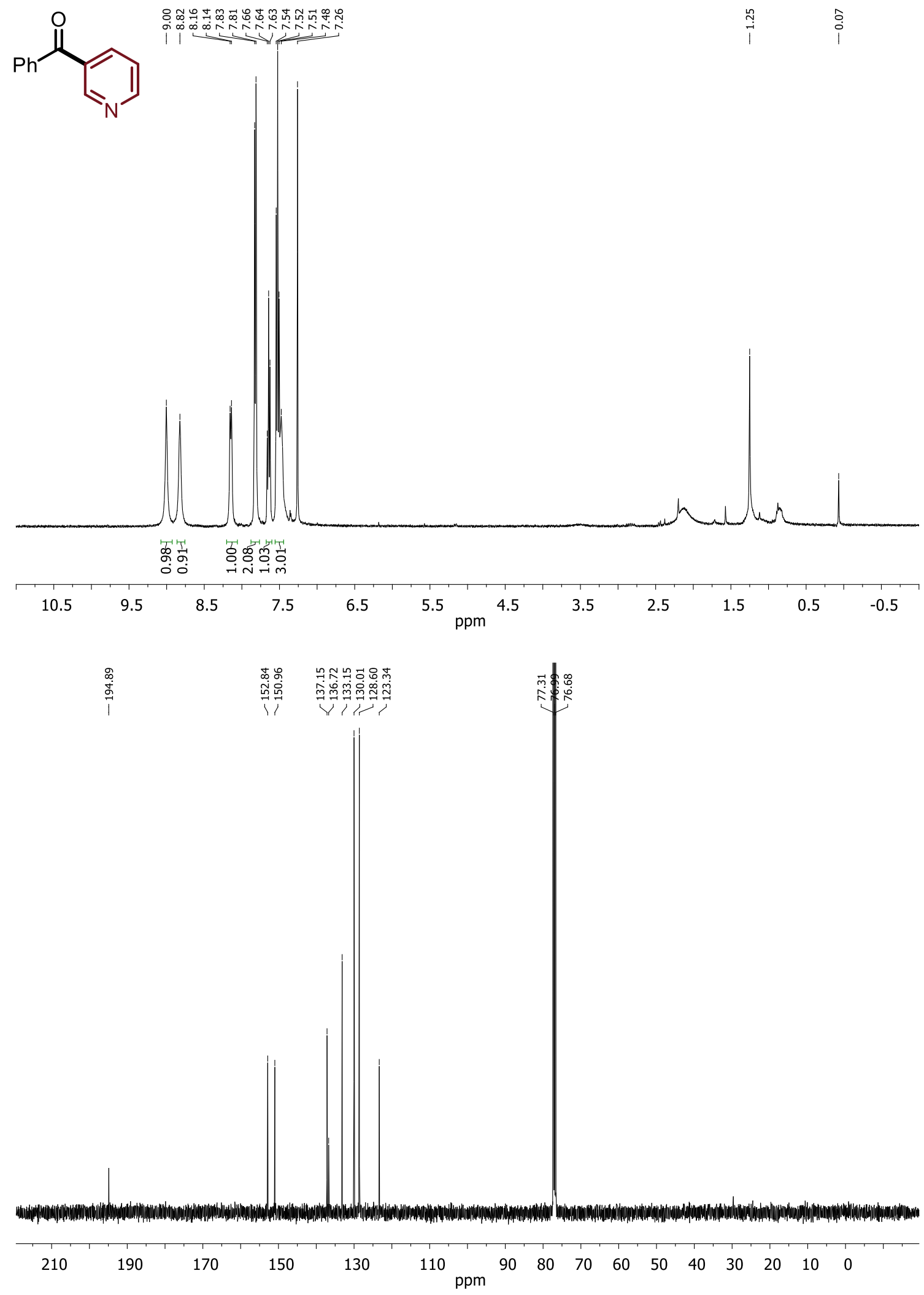
phenyl(quinoxalin-6-yl)methanone (28). $\mathrm{CDCl}_{3}, 400 \mathrm{MHz}$ :
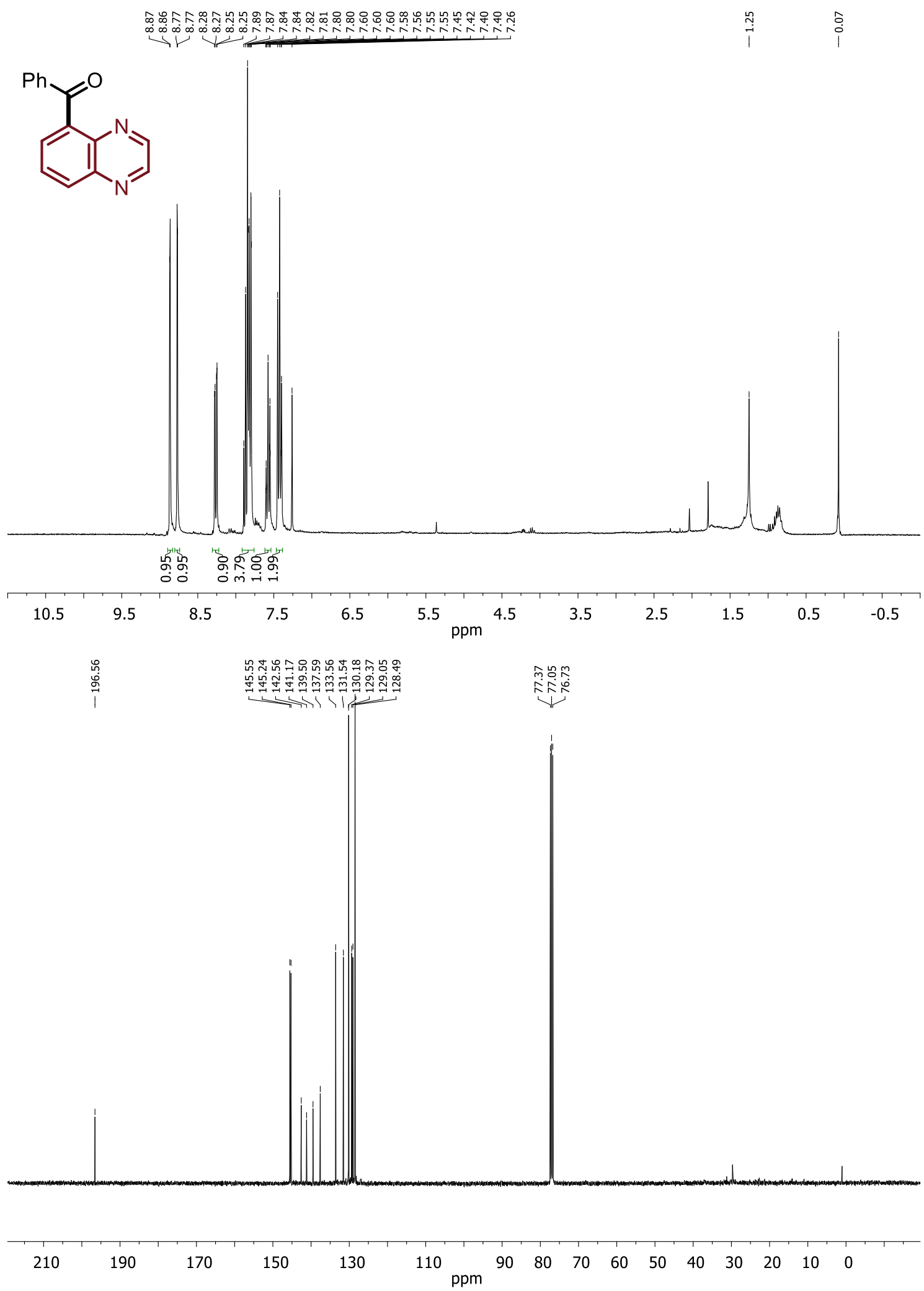
benzo[d]thiazol-6-yl(phenyl)methanone (29). $\mathrm{CDCl}_{3}, 400 \mathrm{MHz}$ :
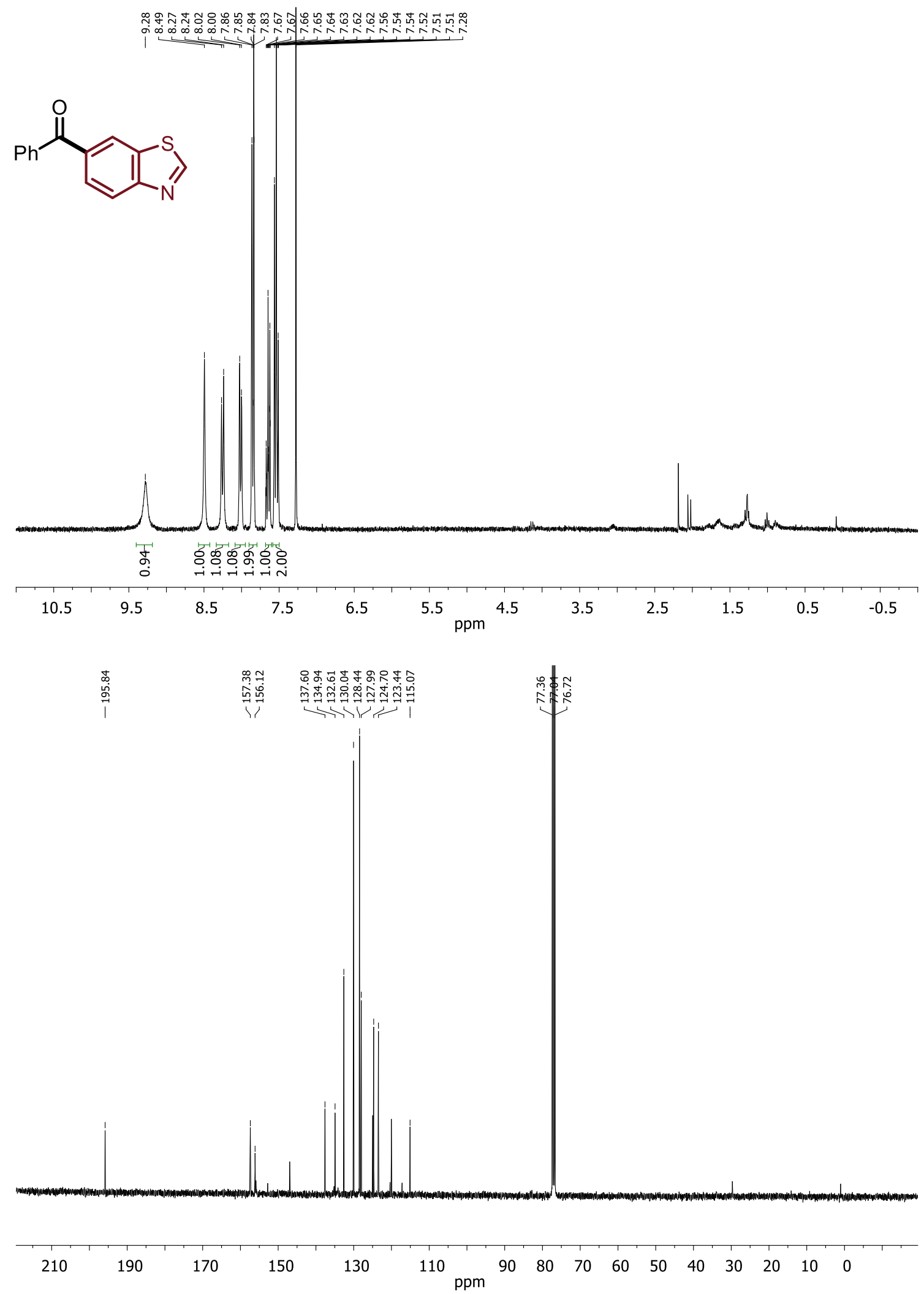
6-chloro-1-phenylhexan-1-one (30). $\mathrm{CDCl}_{3}, 400 \mathrm{MHz}$ :
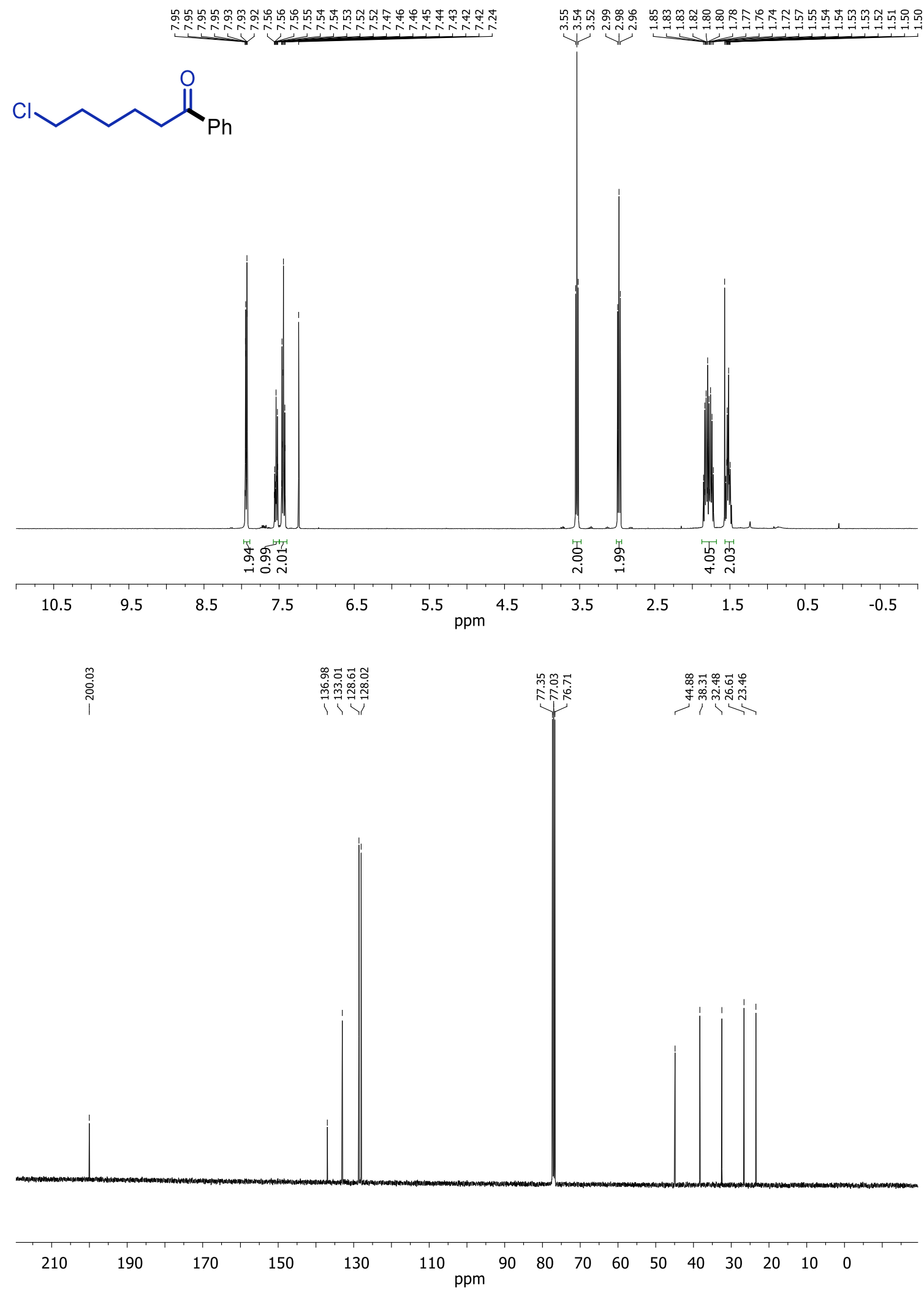
1,3-diphenylpropan-1-one (31). $\mathrm{CDCl}_{3}, 400 \mathrm{MHz}$ :
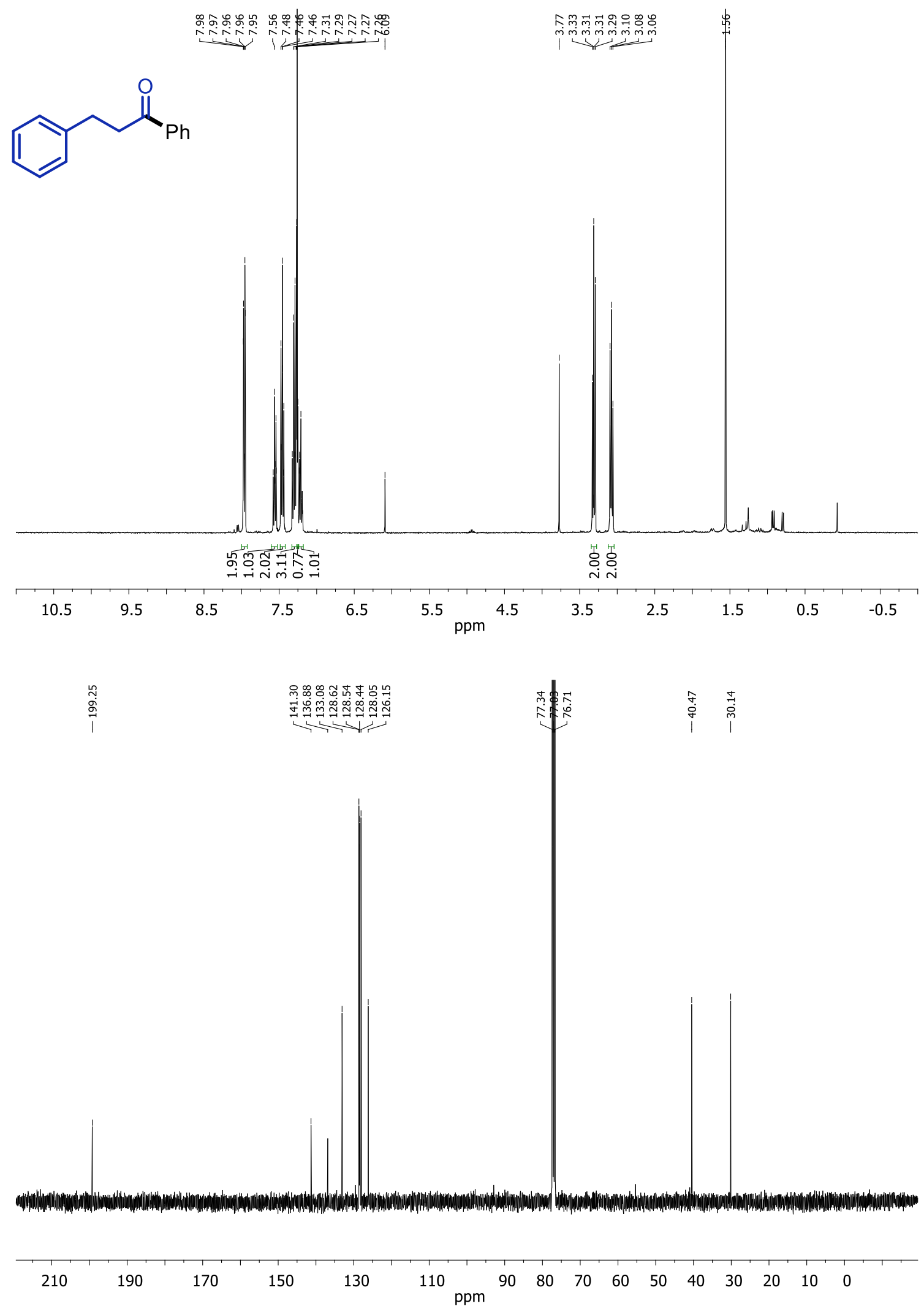
benzoylcyclohexane (32). $\mathrm{CDCl}_{3}, 400 \mathrm{MHz}$ :
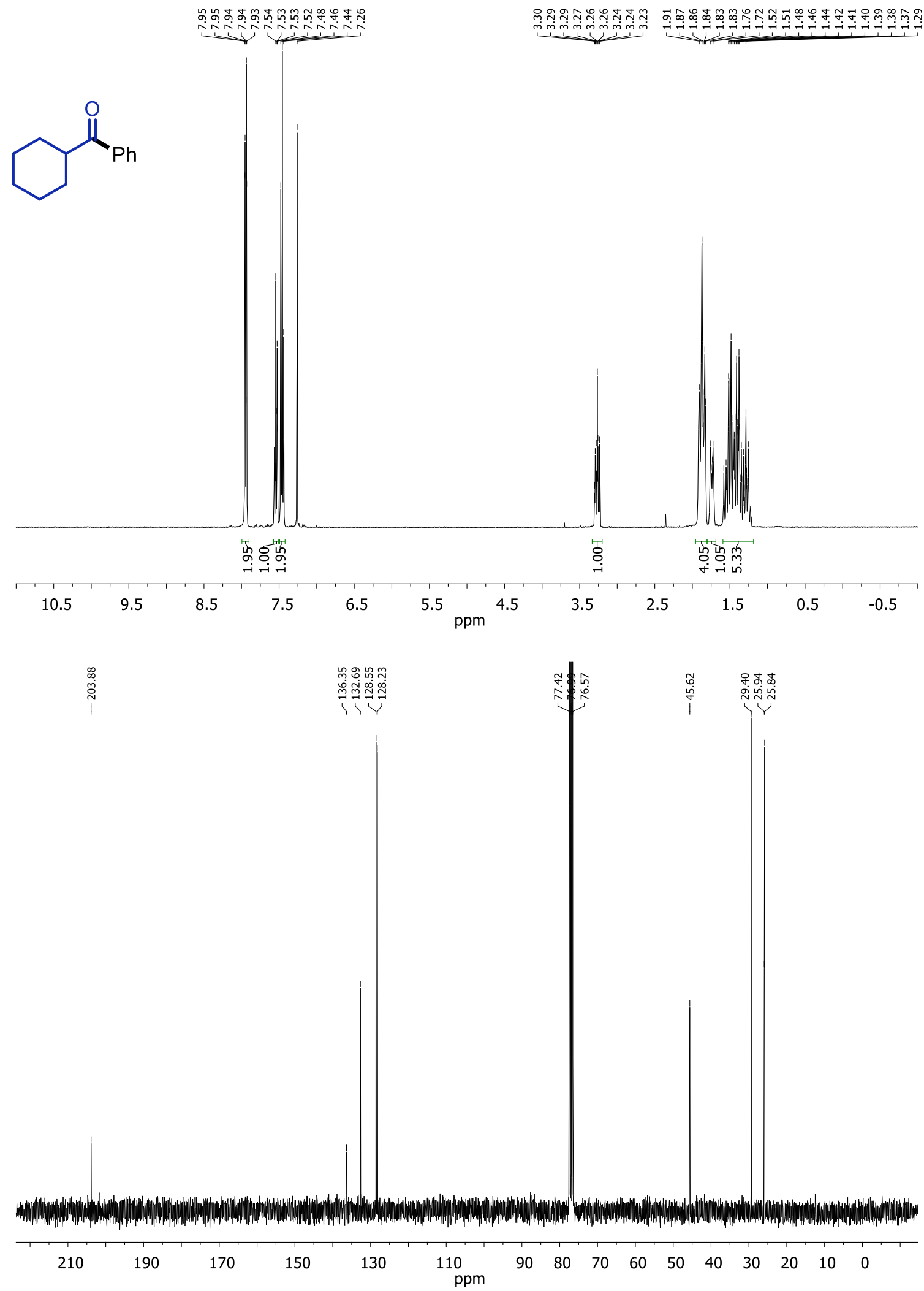
benzoylcyclopropane (33). $\mathrm{CDCl}_{3}, 400 \mathrm{MHz}$ :
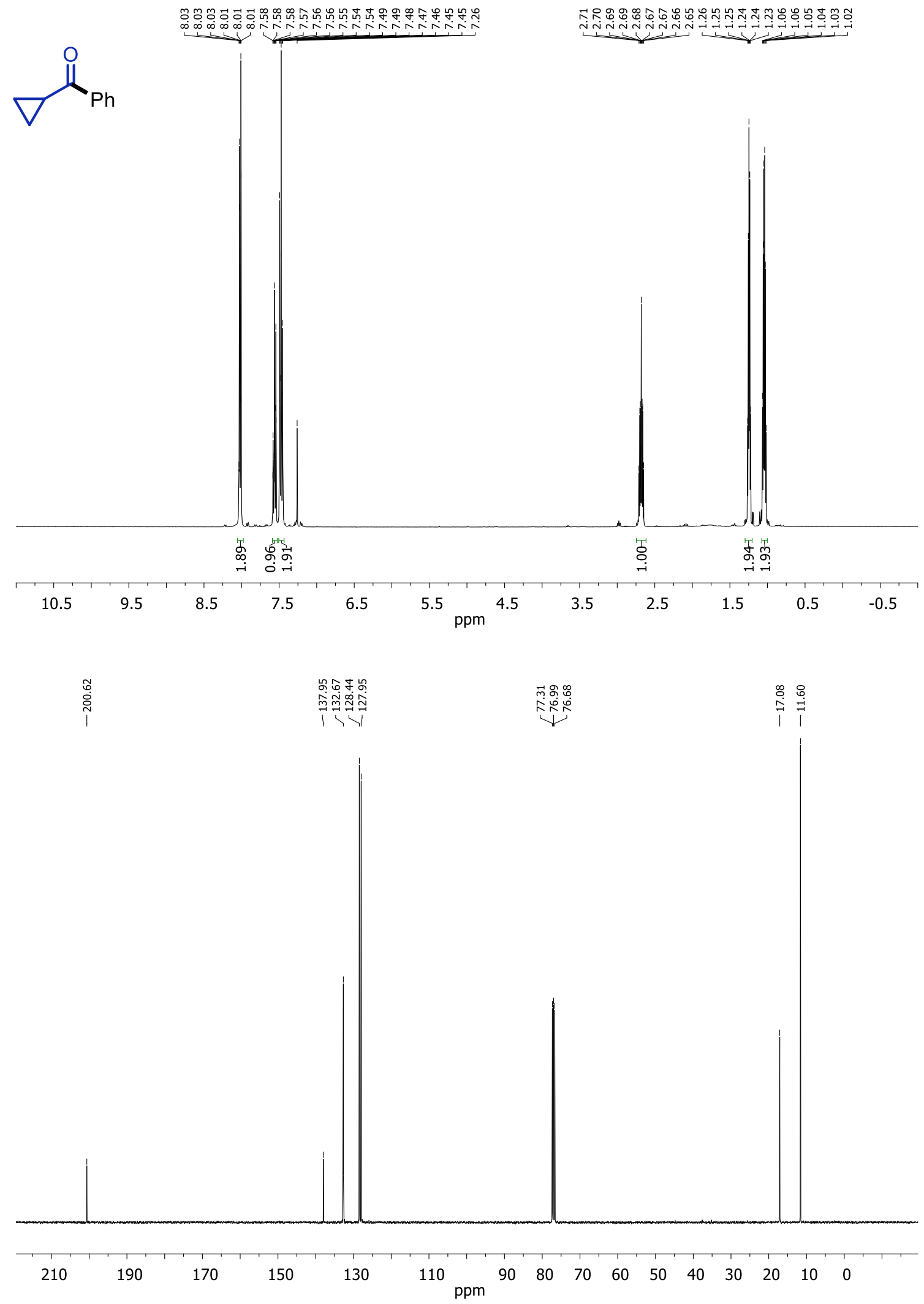
2-(6-methoxynaphthalen-2-yl)-1-phenylpropan-1-one (34). $\mathrm{CDCl}_{3}, 400 \mathrm{MHz}$ :
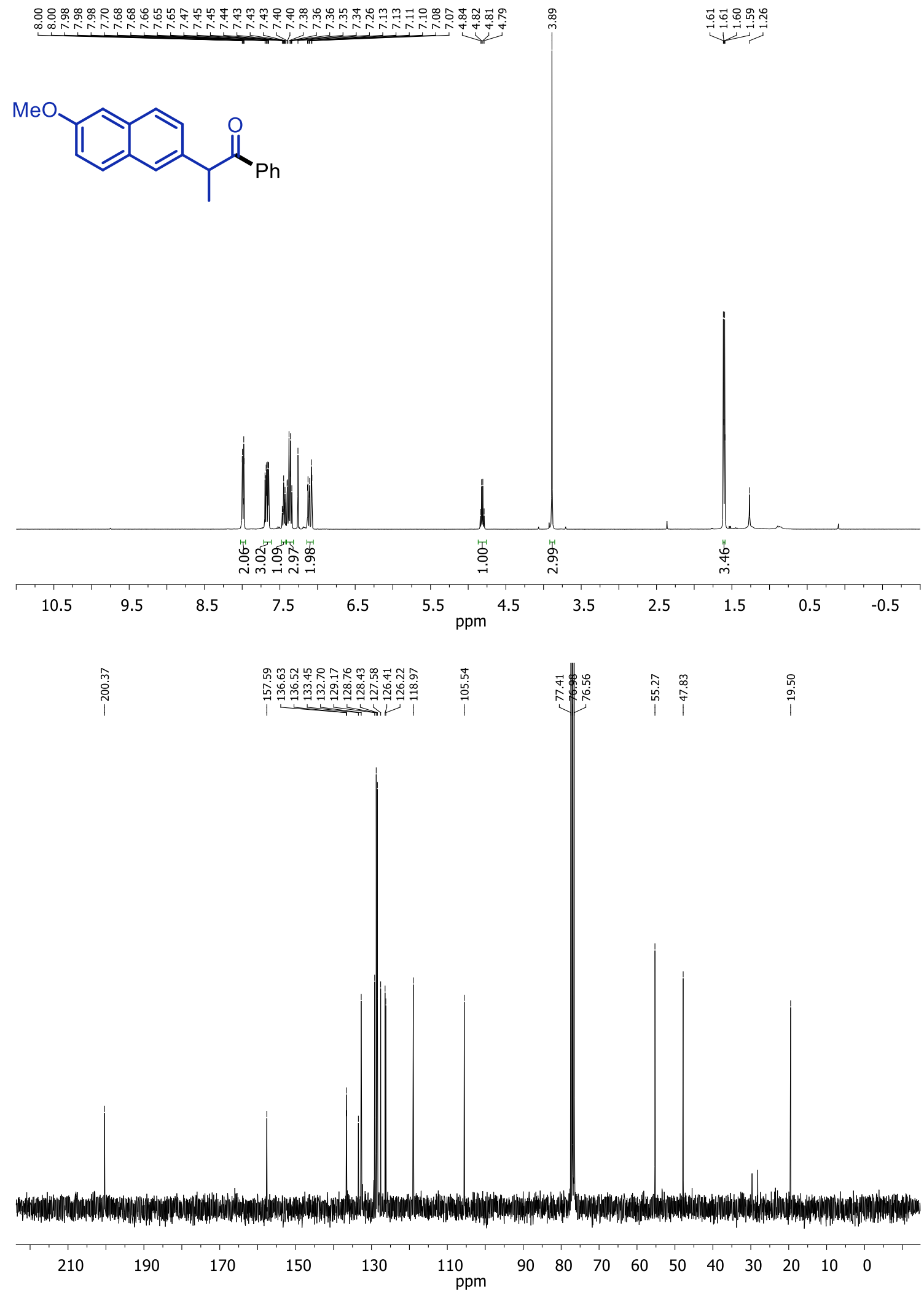
benzoylcyclobutane (35). $\mathrm{CDCl}_{3}, 400 \mathrm{MHz}$ :

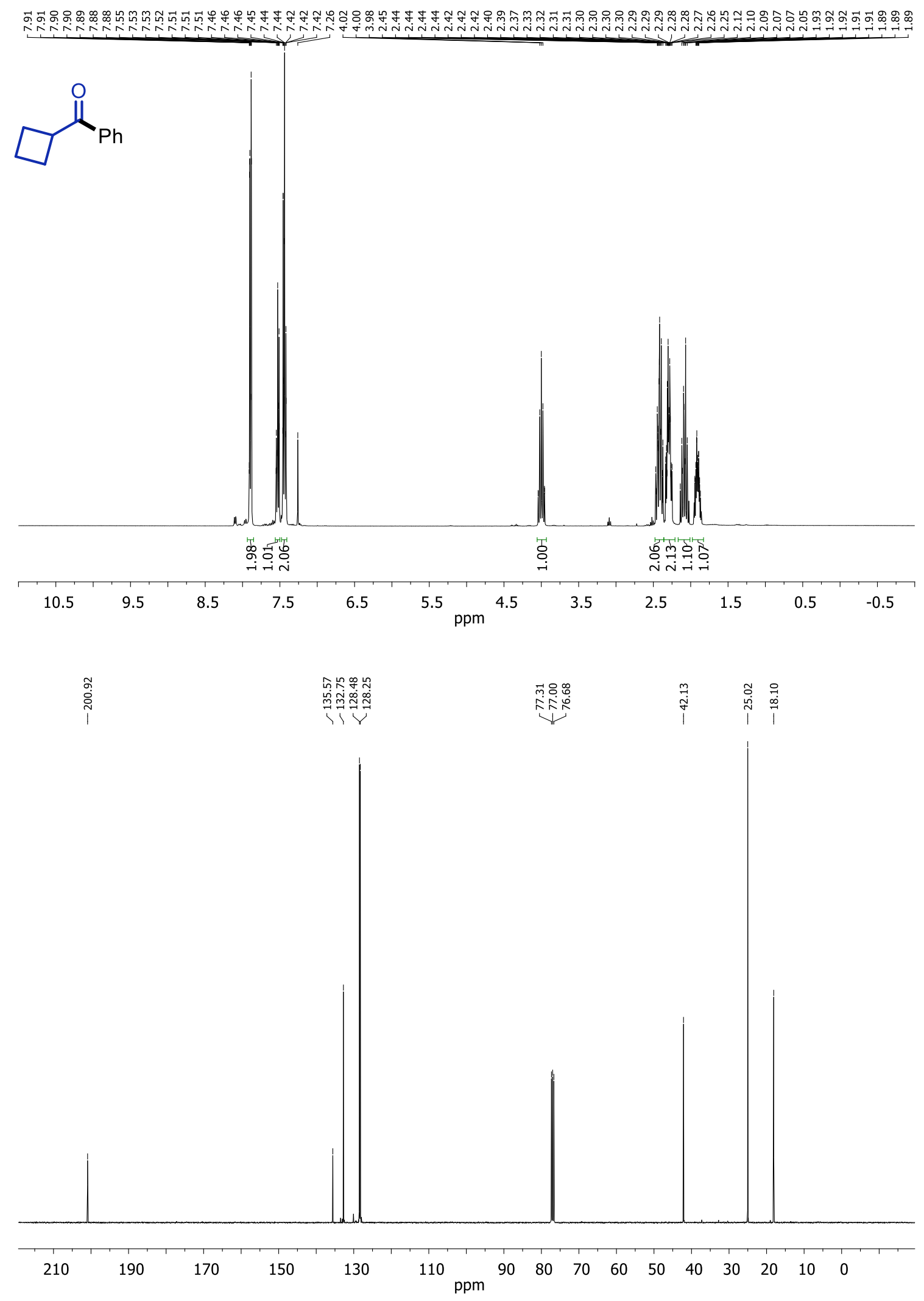


1-adamantyl(phenyl)methanone (36). $\mathrm{CDCl}_{3}, 400 \mathrm{MHz}$ :

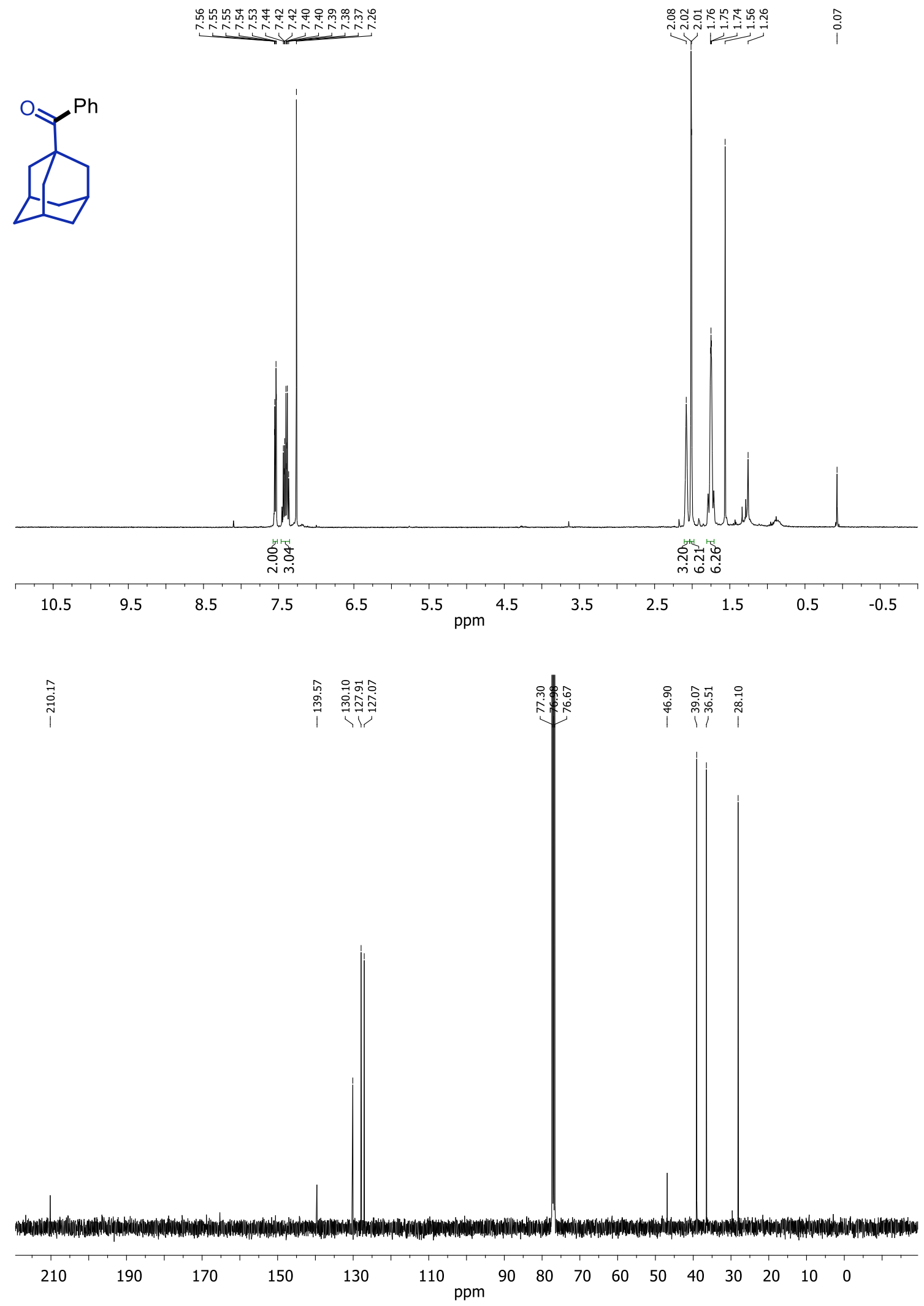


(S)-2-methyl-1-phenylbutan-1-one (37). $\mathrm{CDCl}_{3}, 400 \mathrm{MHz}$ :
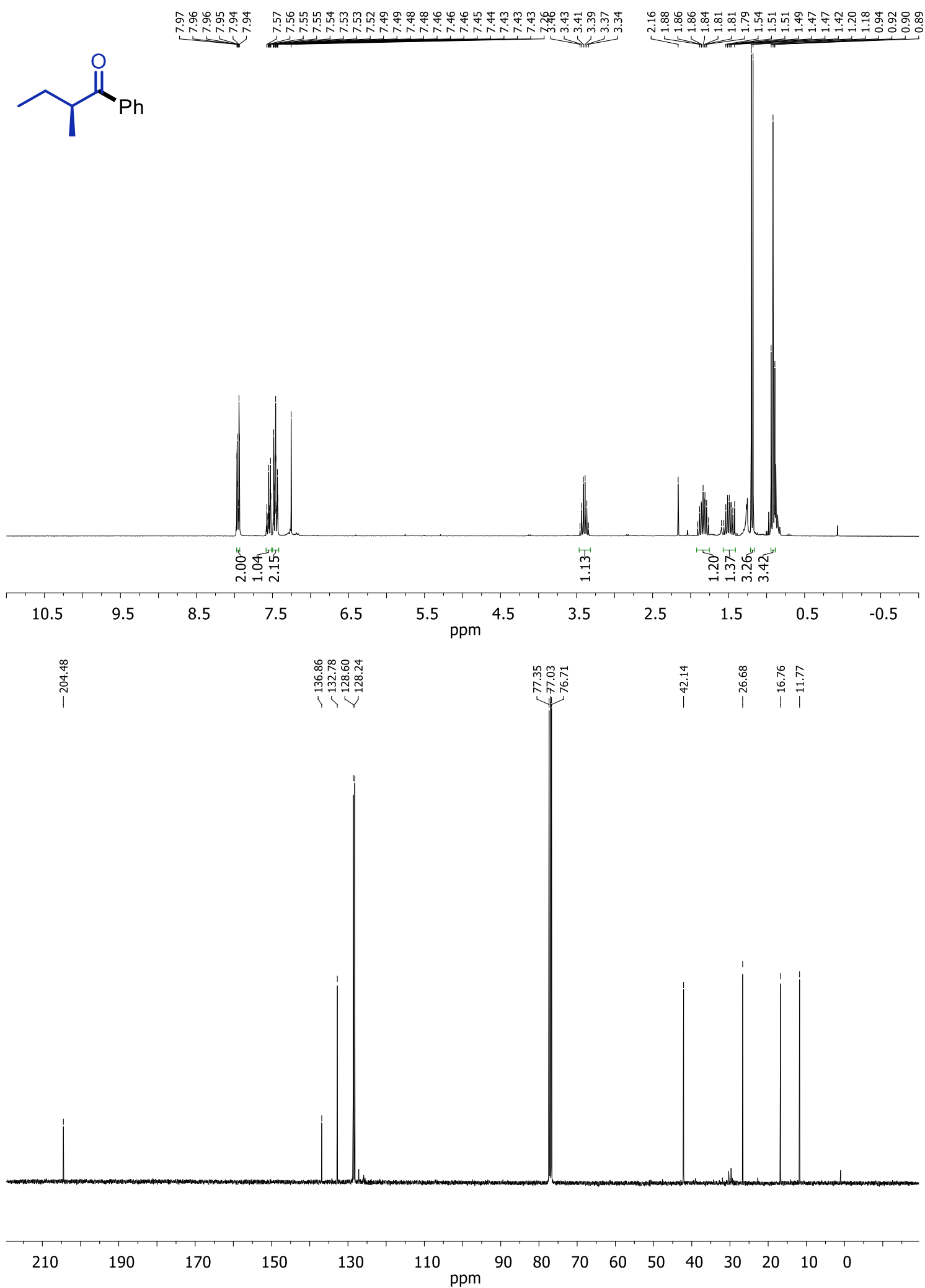
methyl (S)-3-(4-benzoylphenyl)-2-((tert-butoxycarbonyl)amino)propanoate

(38). $\mathrm{CDCl}_{3}, 400 \mathrm{MHz}$ :
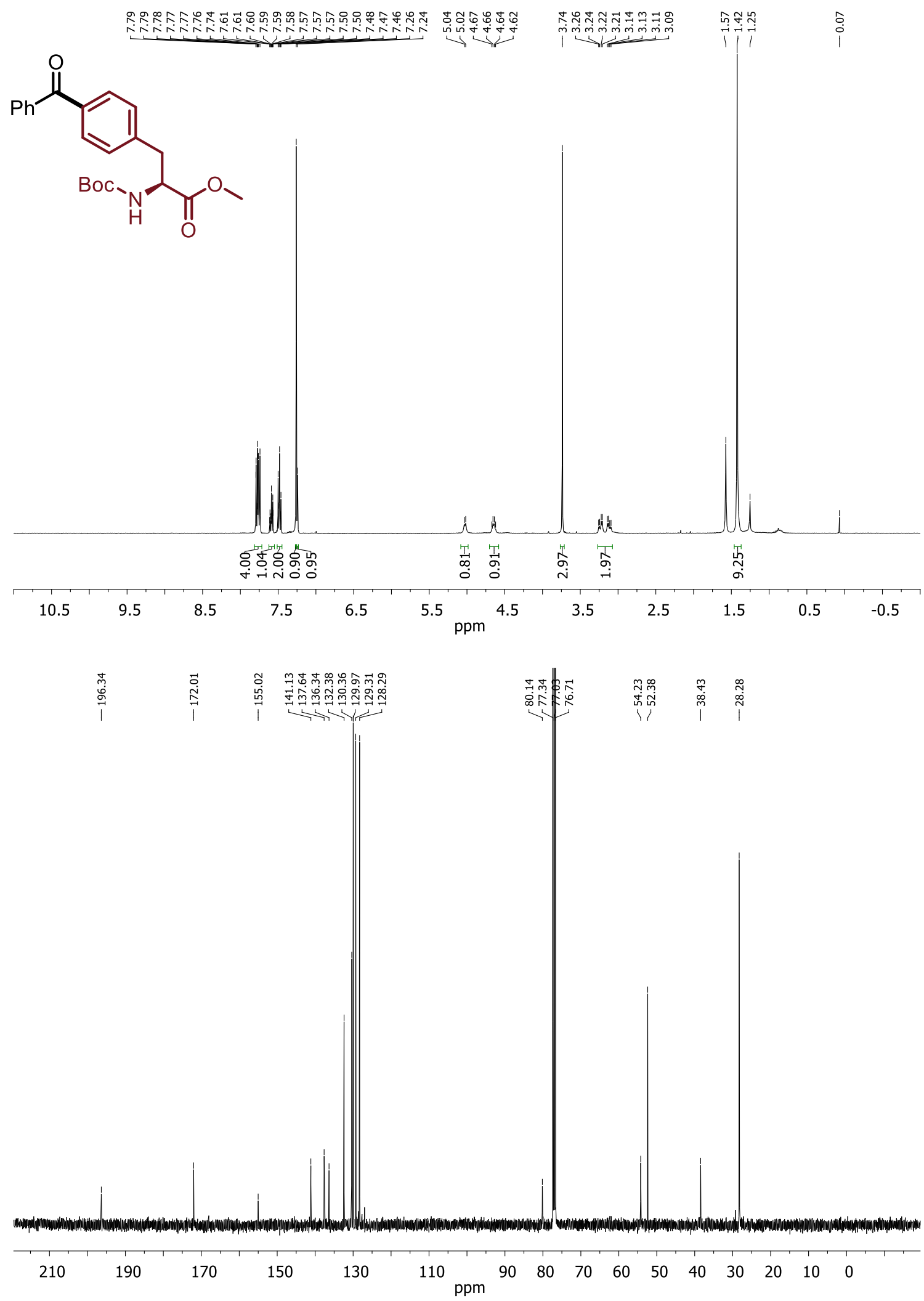
2,3-dimethoxy-5-(10-(4-methoxyphenyl)-10-oxodecyl)-6-methylcyclohexa-2,5-diene1,4-dione (39). $\mathrm{CDCl}_{3}, 400 \mathrm{MHz}$ :

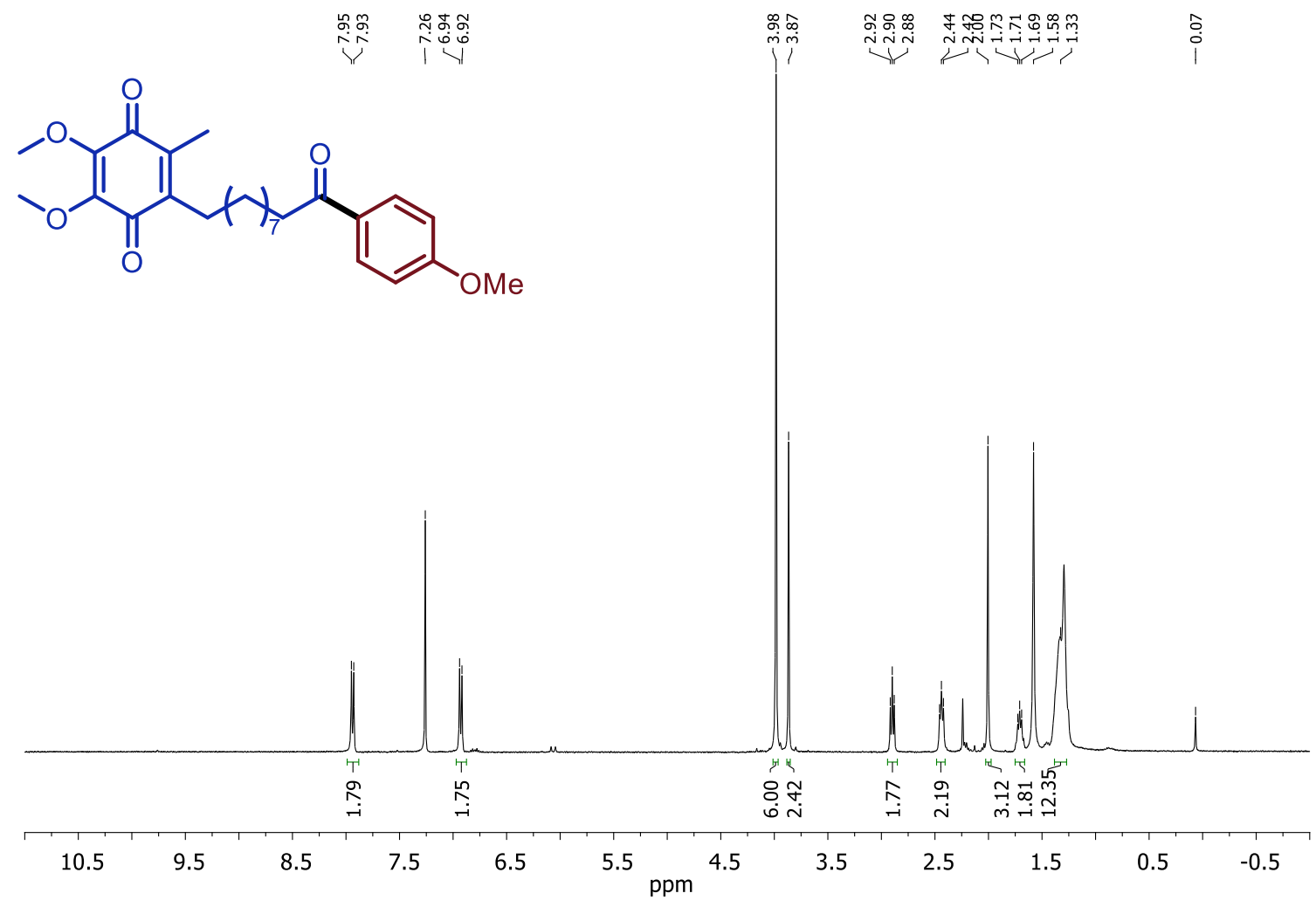

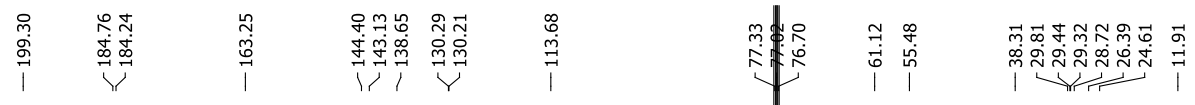

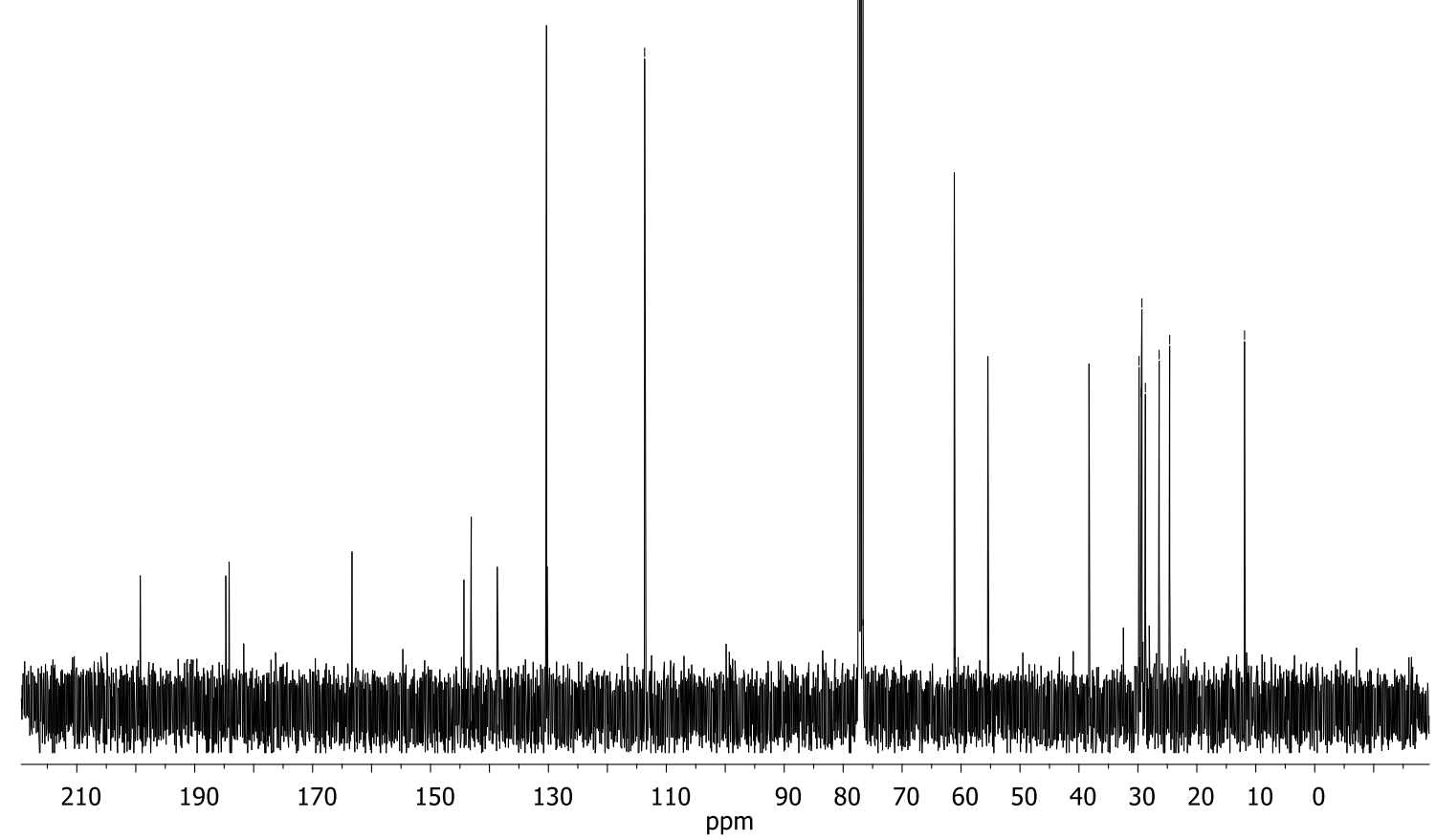


(2-(2,4-dichlorobenzyl)-4-(2,4,4-trimethylpentan-2-yl)phenyl)(p-tolyl)methanone (40). $\mathrm{CDCl}_{3}, 400 \mathrm{MHz}$ :
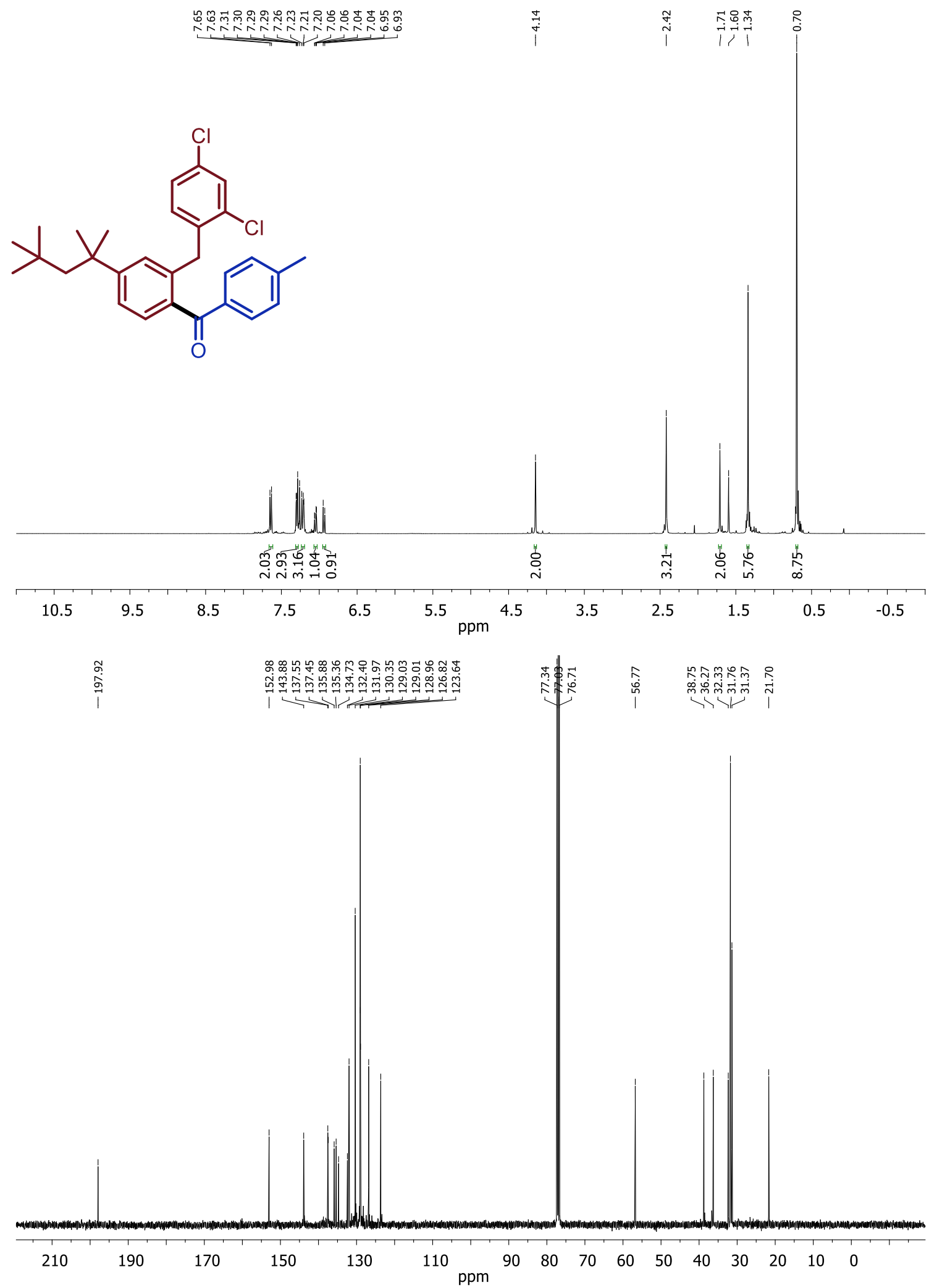
1-(4-fluorophenyl)-4-(4-methylpiperidin-1-yl)butan-1-one (41) (Melperone). $\mathrm{CDCl}_{3}$, $400 \mathrm{MHz}$ :
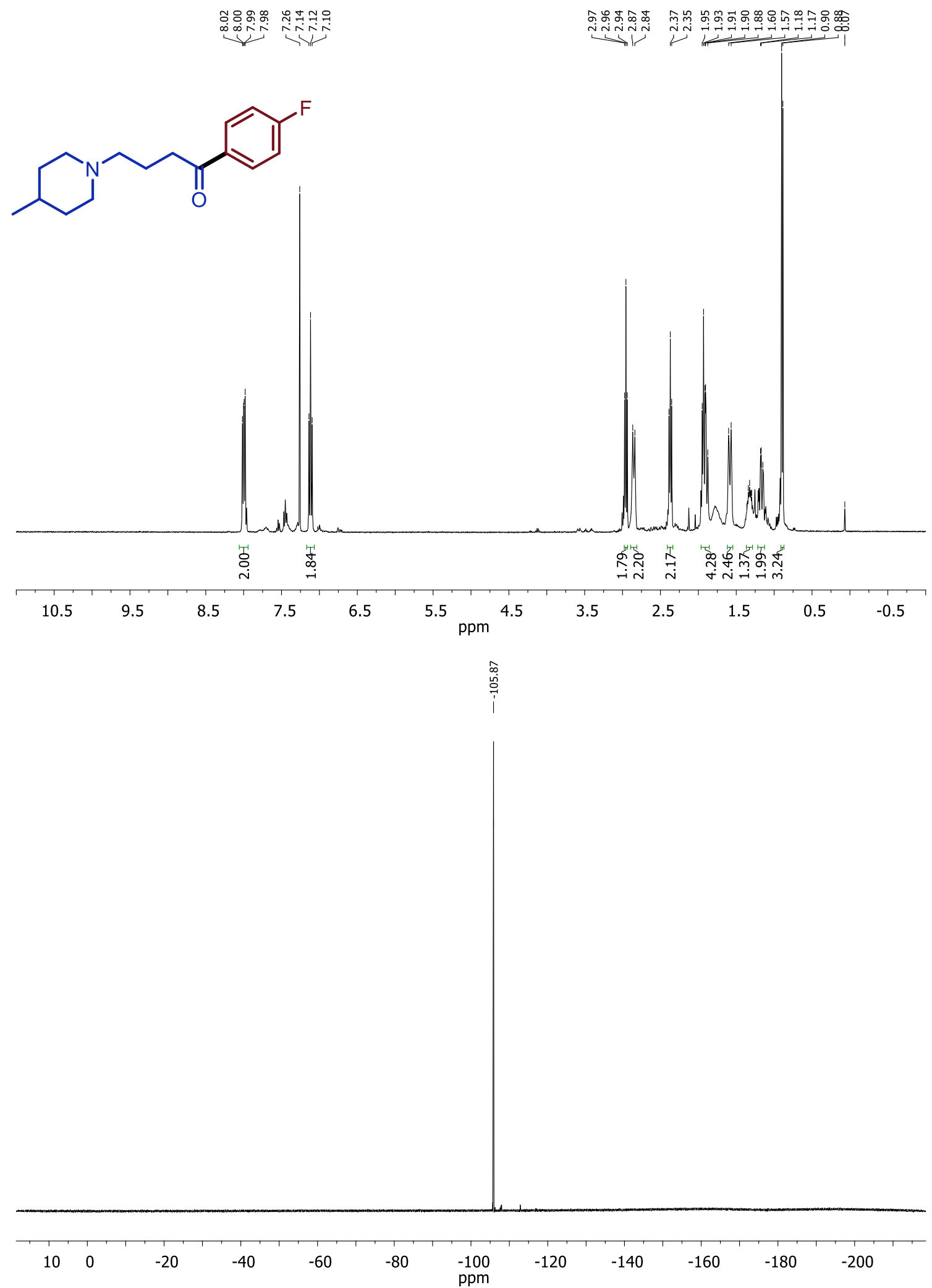


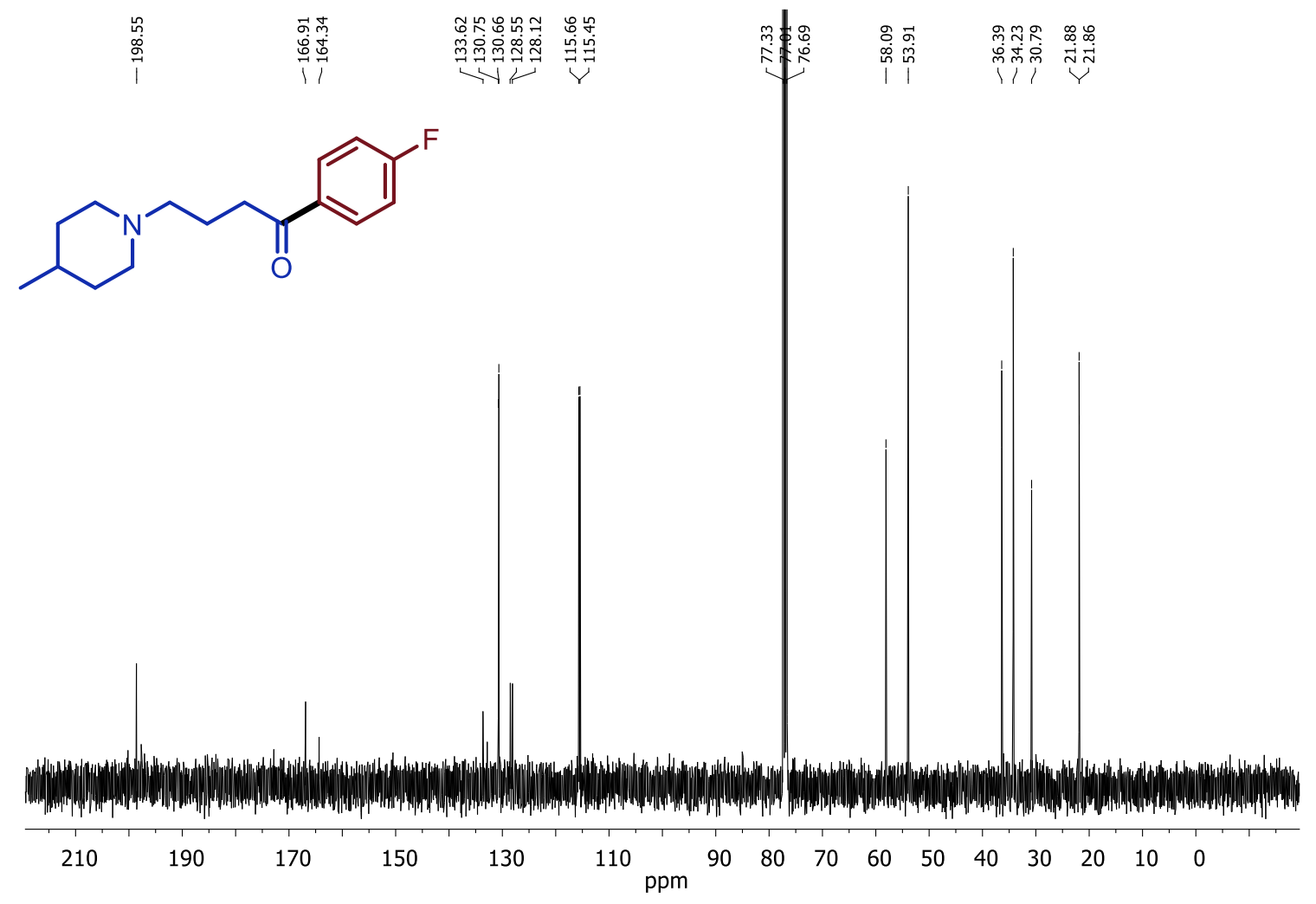


1-(3-fluorophenyl)-4-(4-methylpiperidin-1-yl)butan-1-one (42). $\mathrm{CDCl}_{3}, 400 \mathrm{MHz}$ :
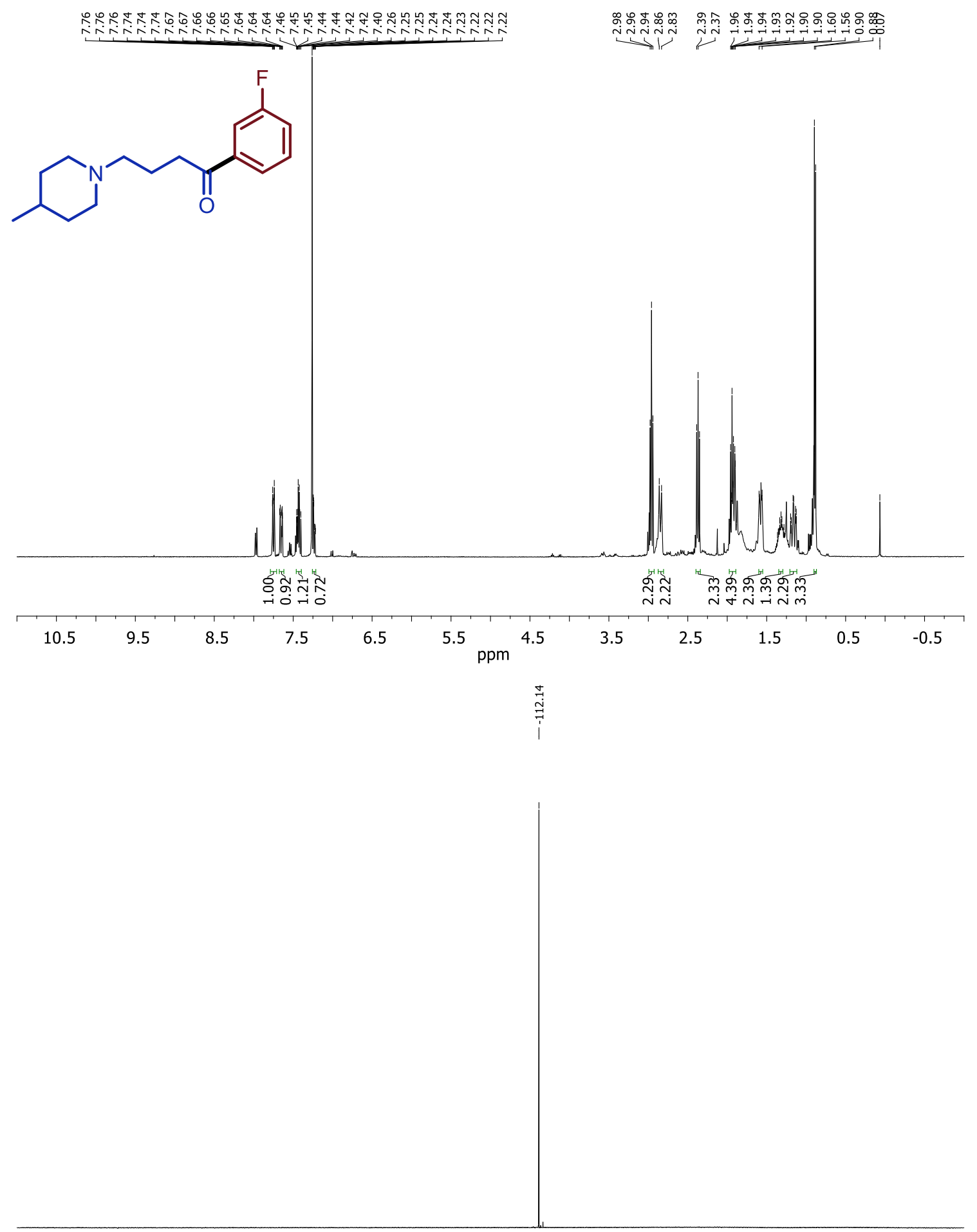

\begin{tabular}{|c|c|c|c|c|c|c|c|c|c|c|c|}
\hline 10 & 0 & -20 & -40 & -60 & -80 & $\begin{array}{l}-100 \\
\mathrm{ppm}\end{array}$ & -120 & -140 & -160 & -180 & -200 \\
\hline
\end{tabular}




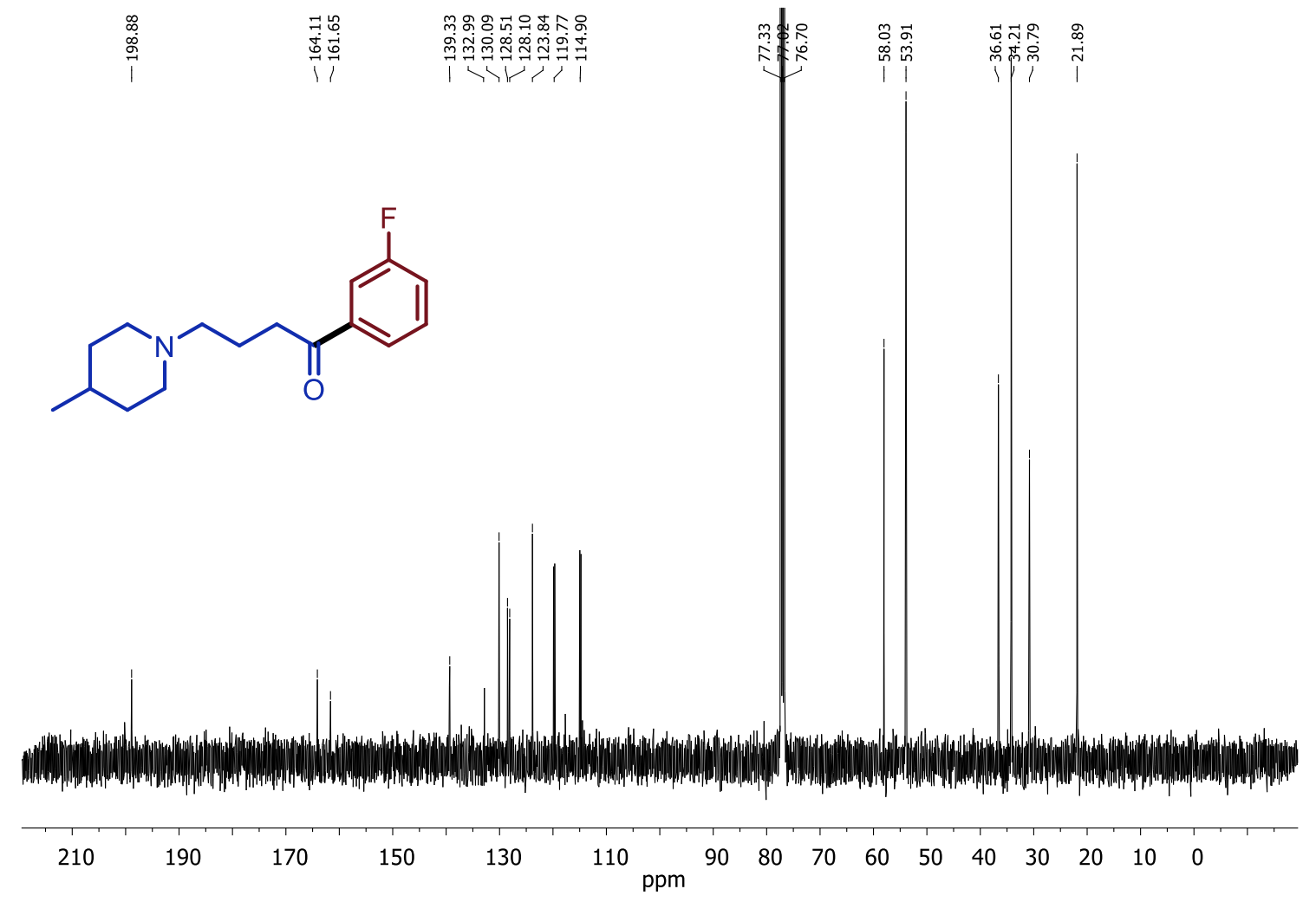


1-(3-fluorophenyl)-4-(4-methylpiperidin-1-yl)butan-1-one (43). $\mathrm{CDCl}_{3}, 400 \mathrm{MHz}$ :
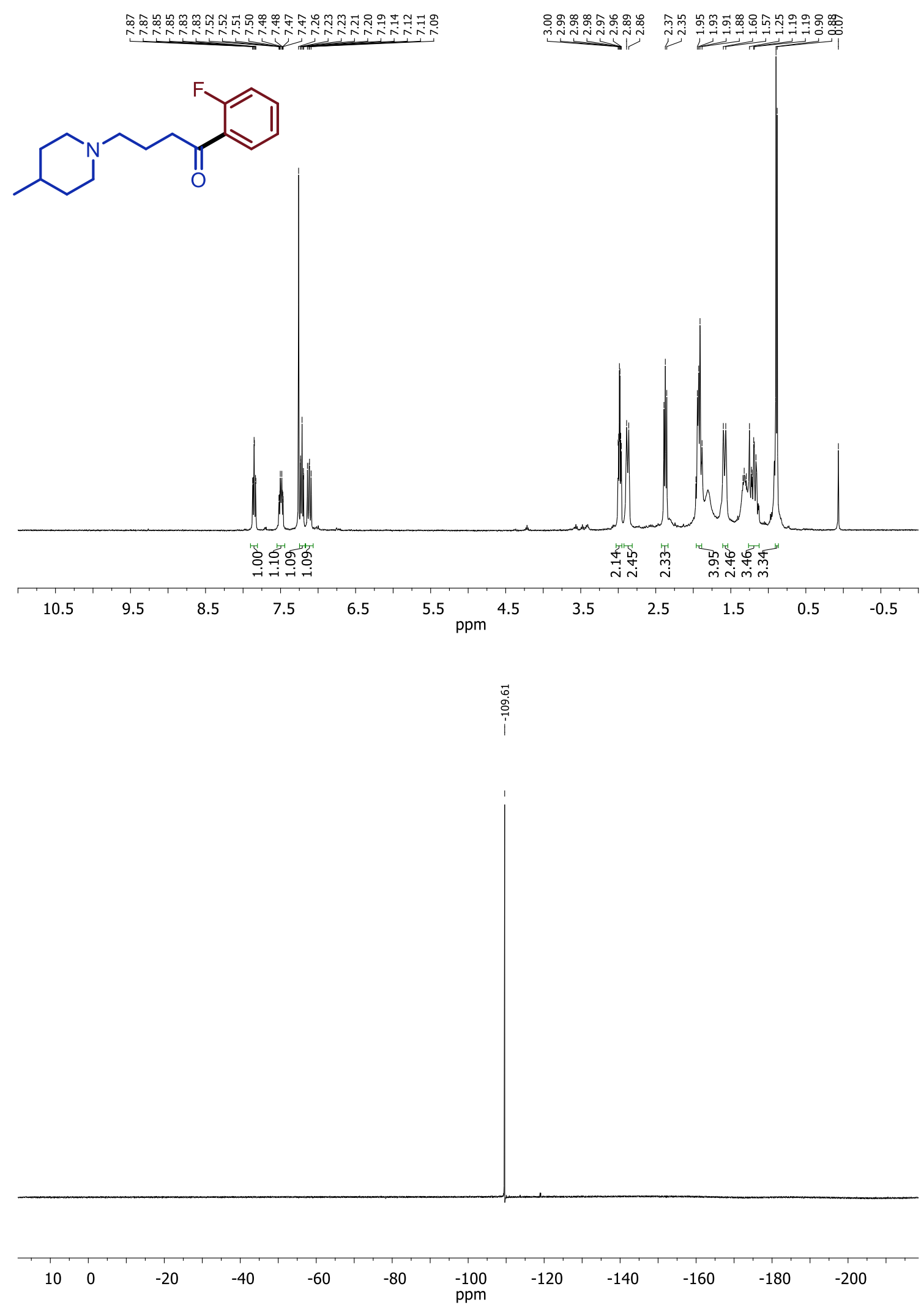


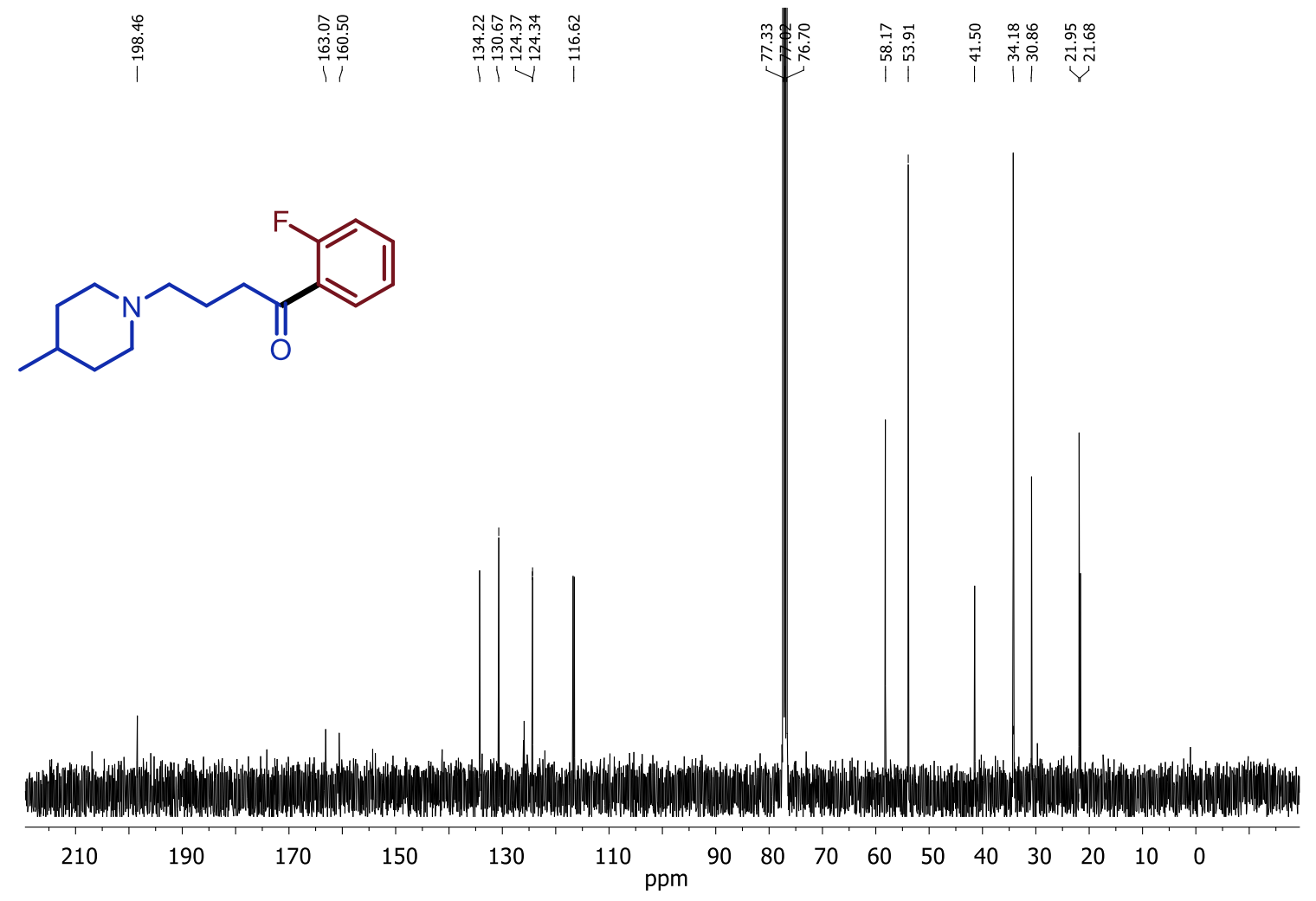


4-(4-(4-chlorophenyl)-4-hydroxypiperidin-1-yl)-1-(4-fluorophenyl)butan-1-one (Haloperidol). $\mathrm{CDCl}_{3}, 400 \mathrm{MHz}$ :
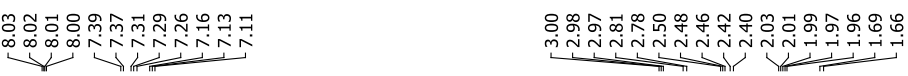

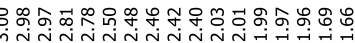
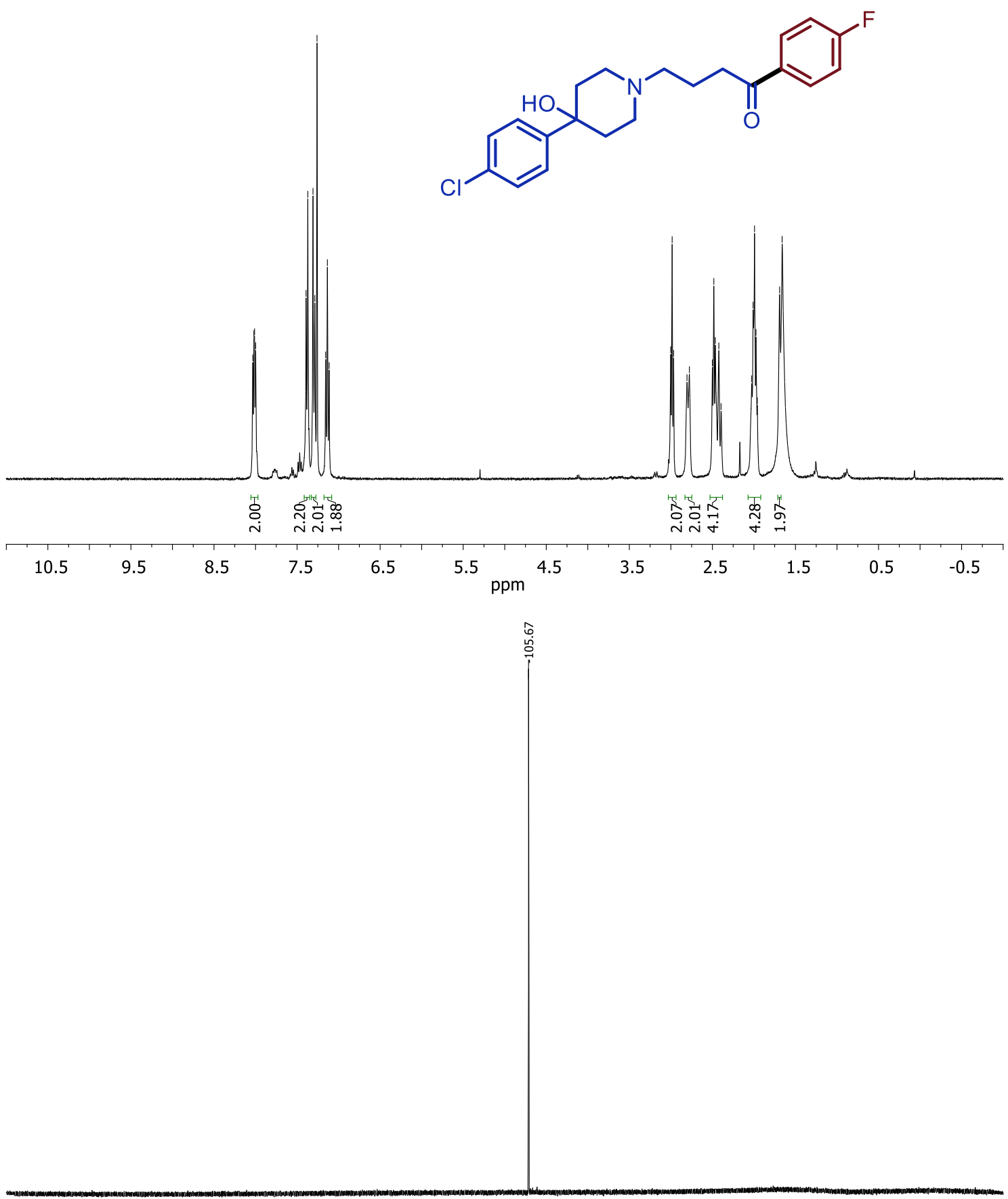

\begin{tabular}{|c|c|c|c|c|c|c|c|c|c|c|c|}
\hline 10 & 0 & -20 & -40 & -60 & -80 & $\begin{array}{l}-100 \\
\mathrm{ppm}\end{array}$ & -120 & -140 & -160 & -180 & -200 \\
\hline
\end{tabular}




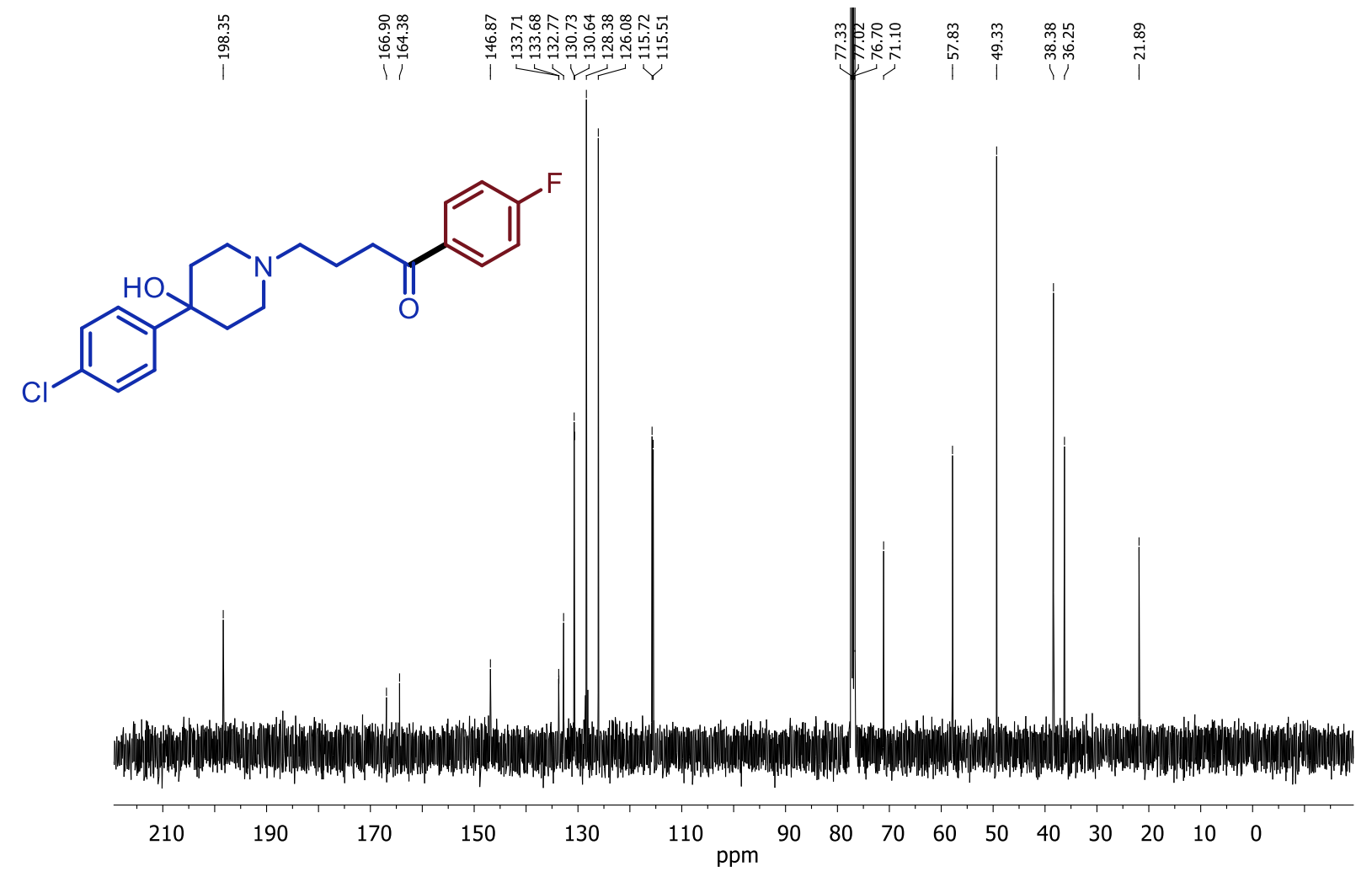




\section{$\underline{\text { 7. References }}$}

(1) Wang, Y.; Gu, M. The Concept of Spectral Accuracy for MS. Anal. Chem. 2010, 82, 70557062.

(2) Shieh, W. C.; Carlson, J. A. A Simple Asymmetric-Synthesis of 4-Arylphenylalanines via Palladium-Catalyzed Cross-Coupling Reaction of Arylboronic Acids With Tyrosine Triflate. $J$. Org. Chem. 1992, 57, 379-381.

(3) Kale, A. P.; Pawar, G. G.; Kapur, M. Regioselectivity Switch Achieved in the Palladium Catalyzed alpha-Arylation of Enones by Employing the Modified Kuwajima-Urabe Conditions. Org. Lett. 2012, 14, 1808-1811.

(4) Vandavasi, J. K.; Hua, X. Y.; Ben Halima, H.; Newman, S. G. A Nickel-Catalyzed CarbonylHeck Reaction. Angew. Chem., Int. Ed. 2017, 56, 15441-15445.

(5) Ben Halima, T.; Zhang, W. Y.; Yalaoui, I.; Hong, X.; Yang, Y. F.; Houk, K. N.; Newman, S. G. Palladium-Catalyzed Suzuki-Miyaura Coupling of Aryl Esters. J. Am. Chem. Soc. 2017, 139, 1311-1318.

(6) Biju, A. T.; Glorius, F. Intermolecular N-Heterocyclic Carbene Catalyzed Hydroacylation of Arynes. Angew. Chem., Int. Ed. 2010, 49, 9761-9764.

(7) Zhong, Y. Z.; Han, W. Iron-catalyzed Carbonylative Suzuki Reactions Under Atmospheric Pressure of Carbon Monoxide. Chem. Commun. 2014, 50, 3874-3877.

(8) Manojveer, S.; Balamurugan, R. Synthesis of Naphthalene Derivatives from orthoAlkynylacetophenone Derivatives via Tandem in situ Incorporation of Acetal and Intramolecular Heteroalkyne Metathesis/Annulation. Org. Lett. 2014, 16, 1712-1715.

(9) O'Keefe, B. M.; Simmons, N.; Martin, S. F. Carbonylative Cross-Coupling of orthoDisubstituted Aryl Iodides. Convenient Synthesis of Sterically Hindered Aryl Ketones. Org. Lett. 2008, 10, 5301-5304.

(10) Pan, S. L.; Zhou, B.; Zhang, Y. H.; Shao, C. D.; Shi, G. F. A Versatile Approach for the Synthesis of para-Substituted Arenes via Palladium-Catalyzed C-H Functionalization and Protodecarboxylation of Benzoic Acids. Synlett 2016, 27, 277-281.

(11) Zhang, M. J.; Tan, D. W.; Li, H. X.; Young, D. J.; Wang, H. F.; Li, H. Y.; Lang, J. P. Switchable Chemoselective Transfer Hydrogenations of Unsaturated Carbonyls Using Copper(I) N-Donor Thiolate Clusters. J. Org. Chem. 2018, 83, 1204-1215.

(12) Maksymenko, S.; Parida, K. N.; Pathe, G. K.; More, A. A.; Lipisa, Y. B.; Szpilman, A. M. Transition-Metal-Free Intermolecular alpha-Arylation of Ketones via Enolonium Species. Org. Lett. 2017, 19, 6312-6315.

(13) Ferles, M.; Kocian, O. Quinoline and Isoquinoline Derivatives. 5. Reduction of 2Quinolinecarbonitrile, 3-Quinolinecarbonitrile and 4-Quinolinecarbonitrile and 3Quinolinecarbonitrile and 4-Quinolinecarbonitrile Methyl Methosulfates with Triethylammonium Formate. Collect. Czech. Chem. Commun. 1979, 44, 2238-2242. 
(14) Wang, J. J.; Zuo, S. J.; Chen, W. Q.; Zhang, X. R.; Tan, K. X.; Tian, Y.; Wang, J. H. Catalytic Formation of Ketones from Unactivated Esters through Rhodium Chelation-Assisted CO Bond Activation. J. Org. Chem. 2013, 78, 8217-8231.

(15) Zhu, X. T.; Ye, C. Q.; Li, Y. J.; Bao, H. L. Iron-Catalyzed Radical Decarboxylative Oxyalkylation of Terminal Alkynes with Alkyl Peroxides. Chem.-Eur. J. 2017, 23, 10254-10258.

(16) Li, X. J.; Zou, G. Acylative Suzuki Coupling of Amides: Acyl-Nitrogen Activation via Synergy of Independently Modifiable Activating Groups. Chem. Commun. 2015, 51, 5089-5092.

(17) Clarke, C.; Fox, D. J.; Pedersen, D. S.; Warren, S. The Lithiation and Acyl Transfer Reactions of Phosphine Oxides, Sulfides and Boranes in the Synthesis of Cyclopropanes. Org. Biomol. Chem. 2009, 7, 1329-1336.

(18) Gonzalez-de-Castro, A.; Xiao, J. L. Green and Efficient: Iron-Catalyzed Selective Oxidation of Olefins to Carbonyls with O-2. J. Am. Chem. Soc. 2015, 137, 8206-8218.

(19) Sumino, S.; Ui, T.; Ryu, I. Synthesis of Alkyl Aryl Ketones by Pd/Light Induced Carbonylative Cross-Coupling of Alkyl Iodides and Arylboronic Acids. Org. Lett. 2013, 15, 3142-3145.

(20) Oppolzer, W.; Darcel, C.; Rochet, P.; Rosset, S.; DeBrabander, J. Non-destructive removal of the bornanesultam auxiliary in alpha-substituted $\mathrm{N}$-acylbornane-10,2-sultams under mild conditions: An efficient synthesis of enantiomerically pure ketones and aldehydes. Helv. Chim. Acta 1997, 80, 1319-1337.

(21) Cheng, W. M.; Shang, R.; Yu, H. Z.; Fu, Y. Room-Temperature Decarboxylative Couplings of alpha-Oxocarboxylates with Aryl Halides by Merging Photoredox with Palladium Catalysis. Chem.-Eur. J. 2015, 21, 13191-13195.

(22) Leyva-Perez, A.; Cabrero-Antonino, J. R.; Rubio-Marques, P.; Al-Resayes, S. I.; Corma, A. Synthesis of the ortho/meta/para Isomers of Relevant Pharmaceutical Compounds by Coupling a Sonogashira Reaction with a Regioselective Hydration. ACS Catal. 2014, 4, 722-731.

(23) Zhang, X. H.; MacMillan, D. W. C. Direct Aldehyde C-H Arylation and Alkylation via the Combination of Nickel, Hydrogen Atom Transfer, and Photoredox Catalysis. J. Am. Chem. Soc. 2017, 139, 11353-11356. 